\title{
Ultrasonic and Hydrothermal Mediated Synthesis Routes for Functionalized Mg-Al LDH: Comparison Study on Surface Morphology, Basic Site Strength, Cyclic Sorption Efficiency and Effectiveness
}

\author{
Collins I. Ezeh ${ }^{\mathrm{a}}$, Marco Tomatis ${ }^{\mathrm{a}}$, Xiaogang Yang ${ }^{\mathrm{a}, *}, \mathrm{Jun} \mathrm{He}^{\mathrm{a}}$, Cheng-gong Sun ${ }^{\mathrm{b}, *}$ \\ ${ }^{\mathrm{a}}$ Department of Mechanical, Materials and Manufacturing Engineering \\ University of Nottingham Ningbo \\ University Park, Ningbo 315100. P.R. China \\ ${ }^{\mathrm{b}}$ Department of Chemical and Environmental Engineering \\ University of Nottingham \\ University Park, Nottingham NG7 2RD, UK
}

\begin{abstract}
Amine functionalized layered double hydroxide (LDHs) adsorbents prepared using three different routes: co-precipitation, sono-chemical and ultrasonic-assisted high pressure hydrothermal. The prepared adsorbent samples were characterised using X-ray diffraction (XRD), X-ray Photoelectron Spectroscopy (XPS), Scanning electron microscope-Energy dispersive X-ray spectroscopy (SEM-EDX), Temperature Programmed Desorption (TPD), Brunauer-Emmett-Teller (BET), and Thermogravimetric analysis (TGA), respectively. The performance of the prepared adsorbents was tested under a controlled thermal-swing adsorption process to measure its adsorption capacity, regeneration and cyclic efficiencies subsequently. The characterisation results were compared with those obtained using the conventional preparation routes but taking the impact of sonochemical and hydrothermal pre-treatment on textural properties, adsorption capacity, regeneration and cyclic efficiencies into account. Textural results depicts a surge in surface area of the adsorbent synthesised by hydrothermal route $\left(311 \mathrm{~m}^{2} / \mathrm{g}\right)$ from 25 and $171 \mathrm{~m}^{2} / \mathrm{g}$ for conventional and ultrasonic routes respectively. Additionally, it has been revealed from the present study that adsorbents prepared using ultrasonic-assisted hydrothermal route exhibit a better $\mathrm{CO}_{2}$ uptake capacity than that prepared using sonochemical and conventional routes. Thus, the ultrasonic-assisted hydrothermal treatment can effectively promote the adsorption capacity of the adsorbent. This is probably due to the decrease of moderate $(\mathrm{M}-\mathrm{O})$ and weak $\left(\mathrm{OH}^{-}\right.$groups) basic sites with subsequent surge in the number of strong basic sites $\left(\mathrm{O}^{2-}\right)$ resulting from the hydrothermal process. Moreover, the cyclic adsorption efficiency of the ultrasonic mediated process was found to be $76 \%$ compared with $60 \%$ for conventional and 53\% for hydrothermal routes, respectively. According to the kinetic model analysis, adsorption mechanism is mostly dominated by physisorption before amine modification and chemisorption after the modification process.
\end{abstract}

Keywords: $\mathrm{CO}_{2}$ Adsorption, Layered Double Hydroxide, Ultrasound, Hydrothermal, Regeneration.

*Corresponding authors:

Tel: +86-574-88182419 E-mail: Xiaogang.Yang@ nottingham.edu.cn (X. Yang)

Tel: +44-115-7484577 E-mail: cheng-gong.sun@nottingham.ac.uk (C. Sun) 


\section{INTRODUCTION}

Adsorption of carbon dioxide is perceived as the most suitable method for Carbon Capture and Storage (CCS) technology $[1,2]$. Extensive studies on various adsorbents used for $\mathrm{CO}_{2}$ uptake has been carried out over the years. Layered double Hydroxides (LDHs) provided comparatively high uptake capacity with numerous catalytic applications [3,4]. Additionally, its ionic interlayered configuration enhances the number of basic sites and provides high contact surface area [4]. LDHs are anionic clay minerals also known as mixed-metal layered hydroxides or hydrotalcites-like compounds. They possess two dimensional structure of layers shaped by trivalent and divalent cations parted by water and anions molecules (see Figure 1) with a general formula:

$$
\left(\mathrm{M}_{1-x}^{2+} M_{x}^{3+}(\mathrm{OH})_{2}\right)^{x+}\left(A_{x / m}^{m-} \cdot n H_{2} O\right)^{x-}
$$

where $\mathrm{M}^{2+}$ and $\mathrm{M}^{3+}$ are divalent and trivalent cations respectively, $\mathrm{A}^{\mathrm{m}-}$ is a compensating anionic charge, $\mathrm{x}$ is the partial substitution of $\mathrm{M}^{2+}$ and $\mathrm{M}^{3+}$, usually within a $\mathrm{M}^{2+} / \mathrm{M}^{3+}$ ratio of 2 and 5 [4$6]$.

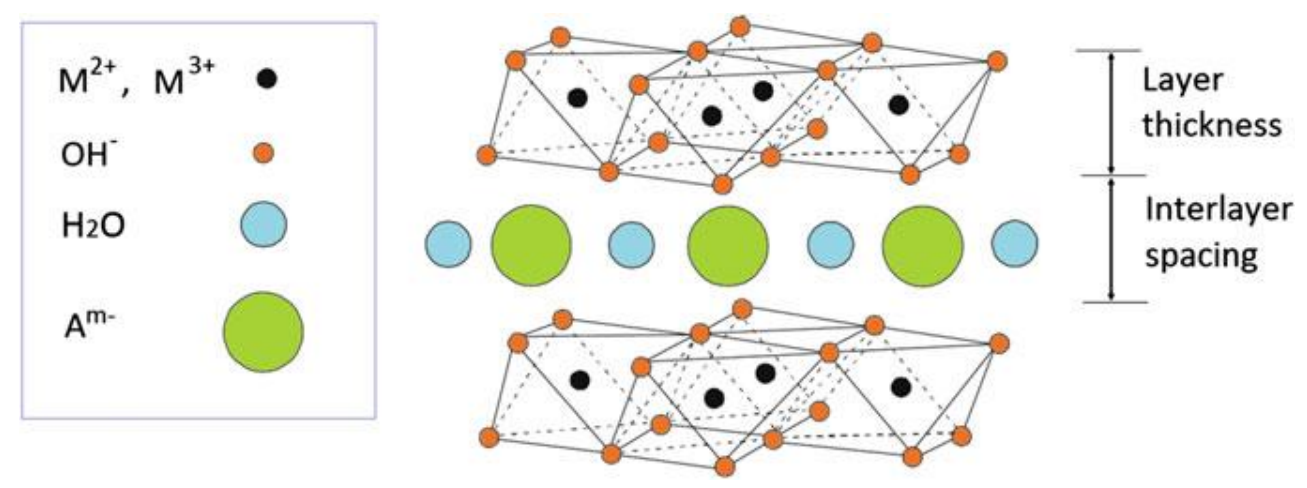

Figure 1: Structural configuration of layered double hydroxides ([5]-permission obtained)

LDHs are basically synthesized via co-precipitation of solutions containing $\mathrm{M}^{2+}$ and $\mathrm{M}^{3+}$ metal salts in a preferred interlayer anion [6,7]. This synthesis method can be conducted at high or low supersaturation environments. In low supersaturation condition, the metal solutions are added concurrently to the solution containing the alkali; whereas the metal (at a given $\mathrm{M}^{2+} / \mathrm{M}^{3+}$ ratio) mixture is added to the alkali and anion precursor solution for high supersaturation conditions. The former tends to produce more crystalline structures because of the increased rate of 
nucleation over crystallization [6]. Besides this method, other means of synthesizing LDH are sol-gel and urea techniques [7,8].

A key property of this material for the purpose of $\mathrm{CO}_{2}$ adsorption and other catalytic reactions is its basic property. Owing to the occurrence of interlayered anions and water molecules in the structure, LDH behave as solid bases. Wide ranges of basic strength can be obtained depending on the treatment route and metallic composition. This is primarily attributed to the nature and deposition of the divalent and trivalent cations on the material. With this property, $\mathrm{CO}_{2}$ has enough acidic strength to bond with the weak basic sites of LDH. However, with an improved basic strength, $\mathrm{CO}_{2}$ is chemisorbed weakly on low basic sites, surface hydroxyl groups forming bicarbonates and strongest on strong basic sites, free lattice oxygen $\left(\mathrm{O}^{2-}\right)$ forming mono-dentate carbonates. Adsorption on medium basic sites are on acid-base (metal-oxygen) pairs like $\mathrm{Mg}^{2+}$ $\mathrm{O}^{2-}$ or $\mathrm{Al}^{3+}-\mathrm{O}^{2-}$ forming tri-dentates or bi-dentates. The use of temperature-programmed desorption monitored by infrared spectroscopy are shown in Figure 2 according to the work [9].

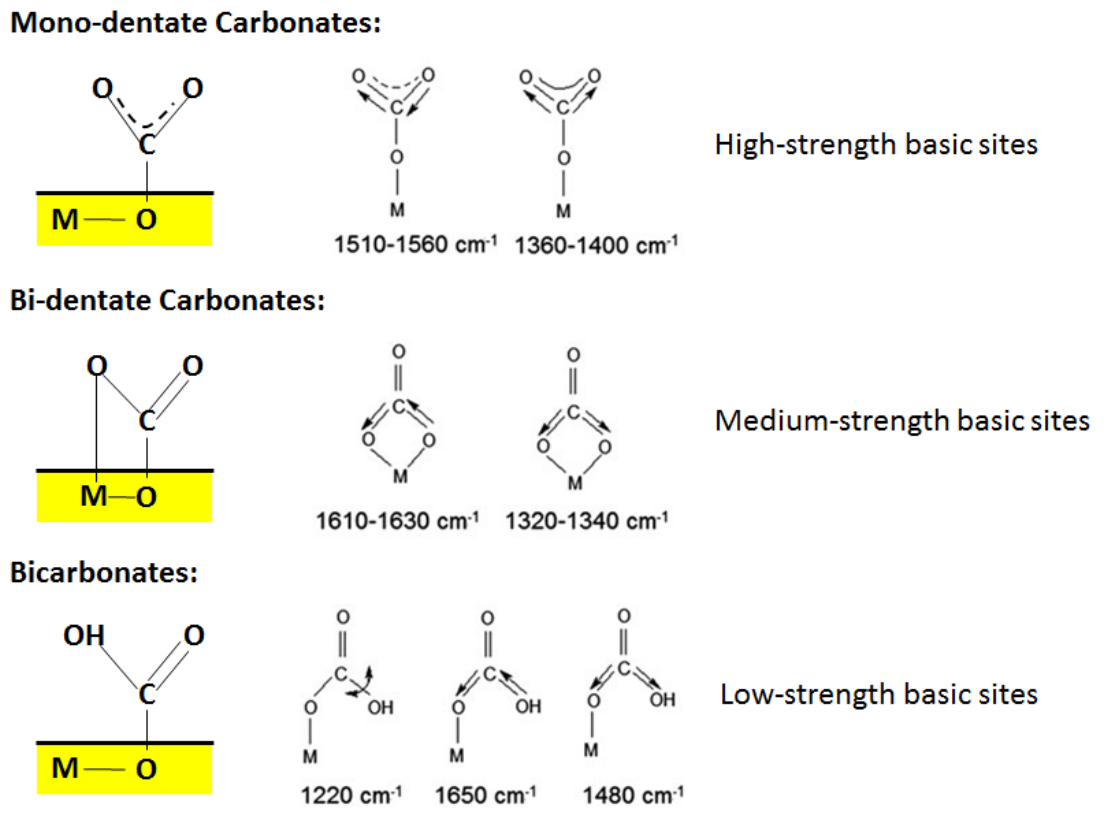

Figure 2: Infrared bands of adsorbed $\mathrm{CO}_{2}$ on LDH surface ([9]-Permission obtained) 
The strength of these basic sites determine the adsorption and regeneration capacity of the adsorbent. Most research studies, to the best our knowledge, have emphasised the effect of metallic composition on this property. However, it is observed from this study that the preparation route plays a key role in the basic strength of this material. Subsequently, this affects the adsorption and regeneration capacities, as well as the life span of the material [10]. In addition, the intimate contact between the metallic contents of the LDHs enhances the physicochemical properties of these materials making it suitable precursors for the synthesis of mixed oxides for hydrogenation and dehydrogenation reactions [6, 11]. Metallic contact can be improved upon by boosting the deposition and dispersion of the cations on the adsorbent. This can be achieved by adopting several preparation routes [3, 12-14].

In this work, the impact of ultrasonic irradiation and ultrasonic-assisted hydrothermal synthesis route on the basicity, adsorption, regeneration and cyclic efficiencies were investigated. This is a follow-up study to recent work [10]. This method has shown to produce materials with wellordered formation [15]. The prepared samples were characterized using Scanning Electron Microscope (SEM), Energy Dispersion X-ray Spectroscopy (EDX), Brunauer-Emmett-Teller (BET), Thermal Gravimetric Analyzer (TGA), Temperature-Programmed Desorption (TPD), Xray Photoelectron Spectroscopy (XPS) and X-ray Diffraction (XRD).

\section{EXPERIMENTAL}

\subsection{Material preparation}

Different preparation methods were used in preparing the layered double hydroxides (LDHs) using the standard method from previous study [10]: conventional co-precipitation, ultrasonic mediated and ultrasonic-assisted hydrothermal. Subsequently, MEA extractions of these LDHs were carried out to produce the amine modified LDHs. All chemicals used for experimentation were acquired from SinoPharm Chemical Reagents Co. Ltd.

\subsection{Sample Synthesis}

For $\mathrm{MgAl} \mathrm{LDH}, 200 \mathrm{ml}$ solution containing APTS ( $\geq 98 \%$ ) and SDS ( $\geq 86 \%$ ) (molar ratio: 5:1) respectively dissolved in a mixture of $50 \mathrm{ml} \mathrm{C}_{2} \mathrm{H}_{5} \mathrm{OH}(\geq 99.7 \%)$ and $150 \mathrm{ml}$ distilled water was stirred for about $30 \mathrm{~min}$ at a temperature of $60{ }^{\circ} \mathrm{C}$ until the $\mathrm{pH}$ stabilized at about 10.3. This 
solution was then reacted with $\mathrm{Mg}\left(\mathrm{NO}_{3}\right)_{2} \cdot 6 \mathrm{H}_{2} \mathrm{O}(\geq 98 \%)$ and $\mathrm{Al}\left(\mathrm{NO}_{3}\right)_{3} \cdot 9 \mathrm{H}_{2} \mathrm{O}(\geq 99 \%)$ (molar ratio: 3:1, dissolved in $100 \mathrm{ml}$ of distilled water) solution by adding the latter dropwise while maintaining the temperature of the former at $60{ }^{\circ} \mathrm{C}$. The mixture $\mathrm{pH}$ was regulated towards 10 using $4 \mathrm{M} \mathrm{NaOH}(\geq 96 \%)$ solution. The substrate (molar ratio of Mg:Al:APTS:SDS = 3:1:5:1) was then aged for $20 \mathrm{hr}$ with the constant stirring at the maintained temperature. The filtered precipitates were then washed with ionised water before being vacuum dried $\left(500 \mathrm{mbar}\right.$ at $\left.70{ }^{\circ} \mathrm{C}\right)$ overnight. This sample is labelled as Conv-LDH. Using the same chemical composition and process, a set of new samples were prepared using sonicated mixing by ultrasonic horns (high intensity sonication, 600W) [10]. This sample is labelled as US-LDH.

For ultrasonic-assisted hydrothermal synthesized samples, HPH-LDH, $32 \mathrm{ml}$ solution containing APTS ( $\geq 98 \%$ ) and SDS ( $\geq 86 \%$ ) (APTS:SDS molar ratio: 5) was reacted with $\mathrm{Mg}\left(\mathrm{NO}_{3}\right)_{2} \cdot 6 \mathrm{H}_{2} \mathrm{O}$ $(\geq 98 \%)$ and $\mathrm{Al}\left(\mathrm{NO}_{3}\right)_{3} \cdot 9 \mathrm{H}_{2} \mathrm{O}(\geq 99 \%) \quad(\mathrm{Mg}: \mathrm{Al}$ molar ratio: 3, dissolved in $16 \mathrm{ml}$ of distilled water) solution. The latter was added dropwise while maintaining the temperature of the former at $60{ }^{\circ} \mathrm{C}$ and sonicating with ultrasonic horn. The substrate (molar ratio of $\mathrm{Mg}$ :Al:APTS:SDS = 3:1:5:1) was moved to the hydrothermal reactor. After being vented with pure $\mathrm{N}_{2}$ gas (99.99\%) for 15 mins in order to remove all of the oxygen before the reaction, the reactor was sealed and heated to a temperature of $450{ }^{\circ} \mathrm{C}$ with a ramp of $2{ }^{\circ} \mathrm{C} / \mathrm{min}$ and then held for 15 mins. After reaction, the solution was aged for $12 \mathrm{hr}$. The precipitates were filtered, washed with distilled water and then dried in a vacuum oven $\left(500 \mathrm{mbar}\right.$ at $\left.70^{\circ} \mathrm{C}\right)$ for $7 \mathrm{~h}$.

For $M g A l L D H-M E A$, the prepared LDHs were functionalised via MEA extraction. $0.5 \mathrm{~g}$ of LDH sample was dispersed in a solution of $100 \mathrm{ml} \mathrm{C}_{2} \mathrm{H}_{5} \mathrm{OH}(\geq 99.7 \%)$ containing $20 \mathrm{~g}$ MEA ( $\left.\geq 99 \%\right)$. The mixture was then refluxed for $20 \mathrm{hr}$ at a temperature of $90{ }^{\circ} \mathrm{C}$. After which the samples were filtered, washed with ethanol and dried in a vacuum oven overnight. These samples are labelled Conv-MEA. Using an ultrasonic horn, the procedure was repeated for the synthesised US-LDHn samples. The obtained samples are labelled US-MEA. Similarly, this was repeated for the HPHLDH samples and labelled as HPH-MEA. 


\subsection{Characterisation}

\subsubsection{Nitrogen adsorption-desorption analysis:}

The textural characteristics of the prepared catalysts were studied by Nitrogen physisorption analysis at $-196{ }^{\circ} \mathrm{C}$ using the Micrometrics ASAP 2020 Surface Area and Porosity Analyser. Prior to this analysis, samples were degassed at a temperature of $105{ }^{\circ} \mathrm{C}$ for $4 \mathrm{hr}$. The surface area $\left(\mathrm{S}_{\mathrm{BET}}\right)$ of the samples was determined using the BET (Brunauer, Emmett and Teller) model. The total pore volumes $\left(\mathrm{V}_{\text {Total }}\right)$ were computed from the amount of nitrogen adsorbed at relative pressure $\left(\mathrm{P} / \mathrm{P}_{\mathrm{o}}\right)$ of 0.99 and the average pore volumes from $4 \mathrm{~V}_{\text {Total }} / \mathrm{S}_{\text {BET }}$. The pore size distribution was calculated using the BJH (Barrett, Joyner and Halenda) model. The t-plot method was used to calculate the micopore volume $\left(\mathrm{V}_{\text {micro }}\right)$.

\subsubsection{Scanning electron microscope-Energy dispersive X-ray spectroscopy (SEM-EDX):}

The surface composition and structure of the prepared materials were studied with a Zeiss $\Sigma_{\text {IGMA }}{ }^{\text {TM }}$ Field Emission SEM and an Oxford Instrument INCAx-act PentaFET® Precision EDX. The EDX spectra for the LDHs were obtained. This was also used to compute the amine content present in the adsorbents

\subsubsection{X-ray Diffraction (XRD) and X-ray Photoelectron Spectroscopy (XPS) Analysis:}

XRD patterns were studied using a Bruker-AXS D8 advance powder diffractometer with a scanning range of $10^{\circ} \leq 2 \theta \leq 90^{\circ}$. X-ray Photoelectron Spectroscopy (XPS) data of the adsorbent was obtained using Kratos X-ray Photoelectron Spectrometer - Axis Ultra DLD with a $96 \mathrm{~W}$ monochromatic $\mathrm{Al} \mathrm{K \alpha}$ X-ray source $(1486.69 \mathrm{eV})$ at a photoelectron take-off angle of $45^{\circ}$. Narrow scans were conducted at steps of $0.05 \mathrm{eV}$ with dwell time of $600 \mathrm{~ms}$ whereas wide scans were conducted from $1100 \mathrm{eV}$ to $0 \mathrm{eV}$ with $150 \mathrm{~ms}$ dwell time and at $1 \mathrm{eV}$ steps. The $\mathrm{C} 1 \mathrm{~s}$ peak at $284.6 \mathrm{eV}$ was used as the reference for standardizing the binding energy.

\subsubsection{Temperature-Programmed Desorption (TPD):}

$\mathrm{CO}_{2}$-TPD analysis was conducted using AutoChem II 2920. The TPD of $\mathrm{CO}_{2}$ measurements were conducted to evaluate the basicity of the catalysts. $0.1 \mathrm{~g}$ of the adsorbent was treated in the reactor in $\mathrm{N}_{2}$ atmosphere at a temperature of $350{ }^{\circ} \mathrm{C}$ for $2 \mathrm{hr}$. A thermal conductivity detector 
(TCD) was adopted to analyze desorption trends from 100 to $800{ }^{\circ} \mathrm{C}$ at a heating rate of $10{ }^{\circ} \mathrm{C} / \mathrm{min}$.

\subsubsection{Thermo-gravimetric analysis:}

Thermal stability and $\mathrm{CO}_{2}$ uptake measurements were conducted using the Netzsch STA 449 F3 Jupiter thermogravimetric analyser (TGA).

Thermal Stability Measurement. The stability of the as-synthesised LDH samples in air was determined. $0.4 \mathrm{~cm}^{3}$ alumina crucible was loaded with $5-10 \mathrm{mg}$ of sample and the thermal decomposition examined from 25 to $1000{ }^{\circ} \mathrm{C}$, ramped $10{ }^{\circ} \mathrm{C} / \mathrm{min}$ and under air flow $(50 \mathrm{ml} / \mathrm{min})$.

$\mathrm{CO}_{2}$ Uptake Measurement. Approximately 5-10 mg of the synthesized sample was heated from 25 to $105{ }^{\circ} \mathrm{C}$ at $20{ }^{\circ} \mathrm{C} / \mathrm{min}$ under $\mathrm{N}_{2}$ at a flow rate of $50 \mathrm{ml} / \mathrm{min}$; and then held isothermally for 30 min before being cooled to the required adsorption temperature at a rate of $10{ }^{\circ} \mathrm{C} / \mathrm{min}$. The gas was switched from $\mathrm{N}_{2}$ to $\mathrm{CO}_{2}$ and held isothermally for $90 \mathrm{~min}$ at a flow rate of $20 \mathrm{ml} / \mathrm{min}$. The experimental adsorption temperature is $55^{\circ} \mathrm{C}$. The $\mathrm{CO}_{2}$ adsorption capacity was calculated from the weight difference of the samples in $\mathrm{CO}_{2}$ atmosphere. The uptake profile was measured against an empty alumina crucible using the same method so as to correct the impact of change in gas density and viscosity.

Thermally Assisted Regeneration of Adsorbent. Regeneration efficiency of the functionalized samples were analysed by thermal-assisted regeneration using nitrogen as a sweep gas. Two cycles of the $\mathrm{CO}_{2}$ uptake measurement was repeated in this case. In between the cycles, the adsorbents were thermally desorbed at $105{ }^{\circ} \mathrm{C}$ in a stream of $\mathrm{N}_{2}$ at a flow rate of $50 \mathrm{ml} / \mathrm{min}$ for 10 mins. The amount adsorbed $\left(\mathrm{Q}_{\mathrm{a}}\right)$ and desorbed $\left(\mathrm{Q}_{\mathrm{d}}\right)$ were deduced by the change in weight of the samples. The regeneration efficiency (RE) was thus estimated based on equation (1):

$$
R E=\frac{Q_{d}}{Q_{a}} \times 100 \%
$$




\section{THEORETICAL}

To predict kinetic behaviour, the experimental results were simulated assuming pseudo-first, pseudo-second order and double-exponential kinetic models [16]. The double exponential model was considered because of its feasibility to explain the surface heterogeneity of the adsorbent accounting for two different adsorption sites. Additionally, it can partially elaborate the kinetic mechanisms associated with both pseudo-first and second order reactions. This mechanism can be categorised into a controlled slow phase intra-particle diffusion and a controlled fast phase surface reaction [17].

\section{Pseudo-first order model}

$$
x=x_{e}\left(1-e^{-k_{f} t}\right)
$$

\section{Pseudo-second order model}

$$
x=\frac{x_{e}^{2} k_{s} t}{1+x_{e} k_{s} t}
$$

\section{$\underline{\text { Double exponential model }}$}

$$
x=x_{e}-\left(A_{1} e^{-k_{1} t}+A_{2} e^{-k_{2} t}\right)
$$

where $x$ and $x_{e}$ represents the $\mathrm{CO}_{2}$ uptake at a given time and equilibrium respectively, $k_{f}$ and $k_{s}$ are the first and second order rate constants respectively, $A_{i}$ and $k_{i}, i=1,2$ are pre-exponential factors and rate constants for the two adsorption sites respectively, and $t$ is the time of adsorption. The obtained experimental data are fitted to the models and selecting the one with the best fit.

To determine the suitability of each model, an error function (Err) defined by equation (5) was applied:

$$
\operatorname{Err}(\%)=\sqrt{\frac{\sum_{j=1}^{N}\left[\frac{x_{j, \exp }-x_{j, \bmod }}{x_{j, \exp }}\right]^{2}}{N-1}} \times 100
$$

where $\mathrm{x}_{\text {exp }}$ and $\mathrm{x}_{\text {mod }}$ are $\mathrm{CO}_{2}$ uptake determined experimentally and computed using the model respectively and $\mathrm{N}$ is the total number of experimental points. It is reasonable to assume that the adsorption rate constant, $\mathrm{k}$ for both pseudo-first and -second order model is the same for each 
group of functionalized and non-functionalized adsorbents since they are both grafted with the same amino silane. However, this was not assumed in this study. The effect of preparation route on the rate constant was rather studied

\section{DISCUSSION OF RESULTS}

\subsection{Impact of preparation route on textural properties and surface morphology}

Table 1: Texture properties of the samples

\begin{tabular}{lcccccc}
\hline \multirow{2}{*}{ Sample } & \begin{tabular}{c}
$\mathbf{S}_{\text {BET }}$ \\
\cline { 2 - 7 }
\end{tabular} & $\begin{array}{c}\text { Particle } \\
\text { Size }\end{array}$ & $\mathbf{V}_{\text {Total }}$ & $\mathbf{V}_{\text {micro }}$ & $\begin{array}{c}\text { Average Pore } \\
\text { Width }\end{array}$ & \% Micro* \\
& $\mathbf{( \mathbf { m } ^ { 2 } / \mathbf { g } )}$ & $\mathbf{( n m )}$ & $\mathbf{( \mathbf { c m } ^ { 3 } / \mathbf { g } )}$ & $\mathbf{( \mathbf { c m } ^ { 3 } / \mathbf { g } )}$ & $\mathbf{( n m )}$ & $\mathbf{( \% )}$ \\
\hline Conv-LDH & 25.03 & 239.67 & 0.0161 & 0.0008 & 2.57 & 4.97 \\
US-LDH & 171.20 & 35.05 & 0.5528 & 0.0229 & 12.92 & 4.14 \\
HPH-LDH & 311.18 & 19.28 & 1.4005 & 0.0023 & 18.00 & 0.16 \\
\hline *\%Micro $=V_{\text {micro }} / V_{\text {Total }}$ & & & & &
\end{tabular}

From Table 1, $\mathrm{S}_{\mathrm{BET}}$ of Conv-LDH is $\mathrm{ca} .25 \mathrm{~m}^{2} / \mathrm{g}$ which showed an increase to 171.20 and 311.18 $\mathrm{m}^{2} / \mathrm{g}$ for US-LDH and HPH-LDH respectively. In the same trend, the total pore volume showed a tremendous increase indicating that the sonication and hydrothermal preparation methods enhances the porosity of the sample as well as the surface area when compared to other studies (see Table 2). However, these methods tend to reduce the micropores in the sample with the hydrothermal route exhibiting the least microporous percentile. This depicts that the sample is a mesoporous/macroporous material with minor microporous features. The features of these pores can be seen from Figure 3. All three samples contained approximately the same volume ratio of mesopores to macropores. However, the Conv-LDH is observed to have a higher percentile of low range mesopores $(2-10 \mathrm{~nm})$ contributing to $55 \%$ of the total pore volume as compared to $10 \%$ and $15 \%$ for US-LDH and HPH-LDH respectively. The high range mesopores $(10-50 \mathrm{~nm})$ accounted for about $50 \%$ of the total pore volume of the US-LDH and HPH-LDH samples compared to $c a$. $7 \%$ in Conv-LDH. This can be further illustrated by the inserted image of Figure 3 displaying the pore size distribution in terms of total volume percentages. 


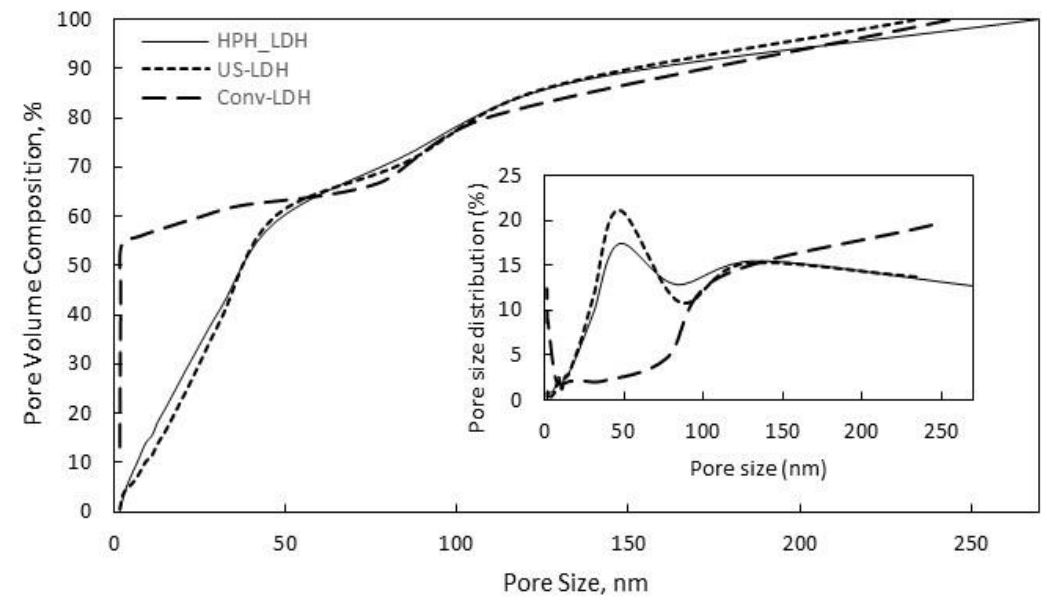

Figure 3: Pore size distribution of Conv-, US- and HPH-LDH samples

Figure 4 shows the total absolute volume of the pores at various pore size. It depicts that the high range mesopores mostly contribute to the total pore volume of the samples and comprises of a higher pore distribution.

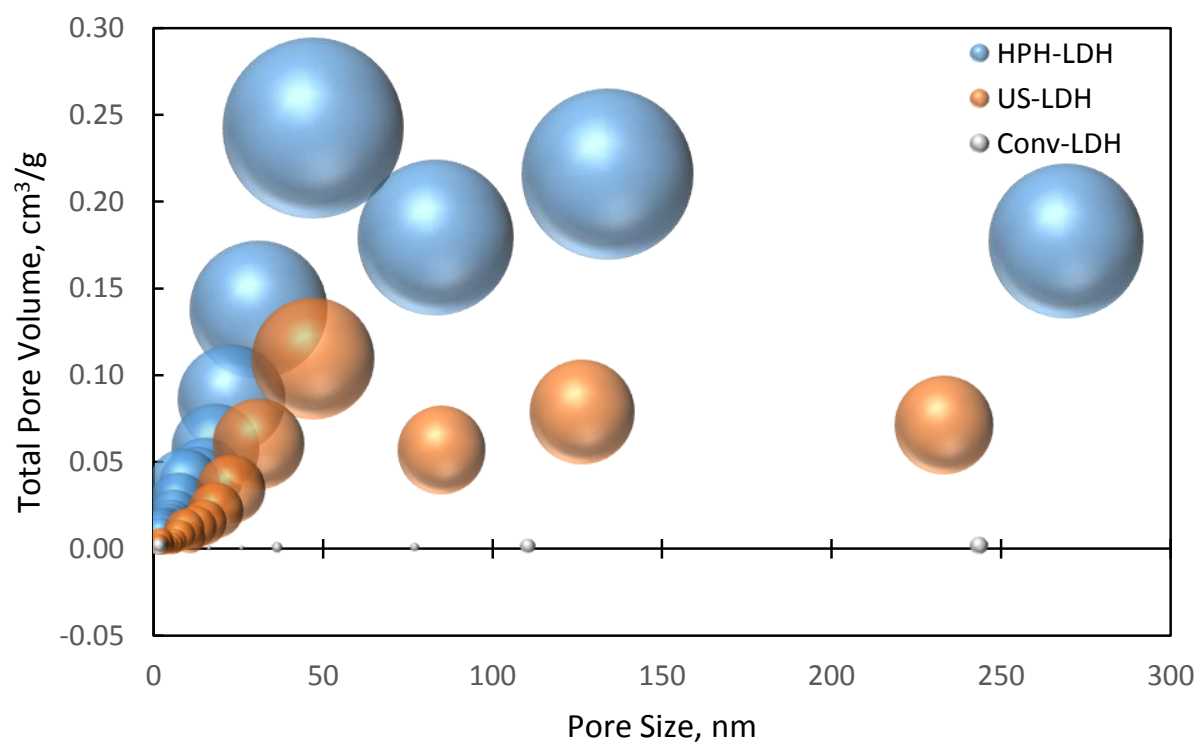

Figure 4: Total pore volume of various pore sizes of Conv-, US- and HPH-LDH samples (bubble size indicates relative amount of pore size). 
Table 2: Comparison of $S_{B E T}$ for different $L D H$ adsorbent via different preparation routes

\begin{tabular}{r|lrr}
\hline \multirow{2}{*}{ Sample* } & Preparation Method & \begin{tabular}{r} 
SBET \\
\cline { 2 - 3 }$\left(\mathbf{m}^{2} / \mathbf{g}\right)$
\end{tabular} & Reference \\
\hline$C O_{3}-L D H$ & Commercial & 2.90 & {$[18]$} \\
$C o n v-L D H$ & Commercial & 18.90 & {$[19]$} \\
$C o n v-L D H$ & Co-precipitation & 10.50 & {$[20]$} \\
$C o n v-L D H$ & Co-precipitation & $44-70$ & {$[18]$} \\
$C o n v-L D H$ & Co-precipitation & 69.78 & {$[21]$} \\
$c-L D H$ & Calcined Co-precipitation & $84-123$ & Current work \\
$c-C O_{3}-L D H$ & Calcined Solvothermal & 165.07 & {$[18]$} \\
$U S-L D H$ & Ultrasonic assisted & 84.44 & {$[22]$} \\
$U S-L D H$ & Ultrasonic assisted & 171.20 & {$[21]$} \\
$r-L D H$ & Reconstructed & $45-55$ & Current work \\
$C O_{3}-L D H$ & Solvothermal & 24.74 & {$[22]$} \\
$L D H$ & Silylated-Calcined & 19.20 & Current work \\
$H P H-L D H$ & Hydrothermal & 311.18 & \\
\hline
\end{tabular}

*LDH samples are $\mathrm{Mg}$ - $\mathrm{Al} \mathrm{LDH}$ 


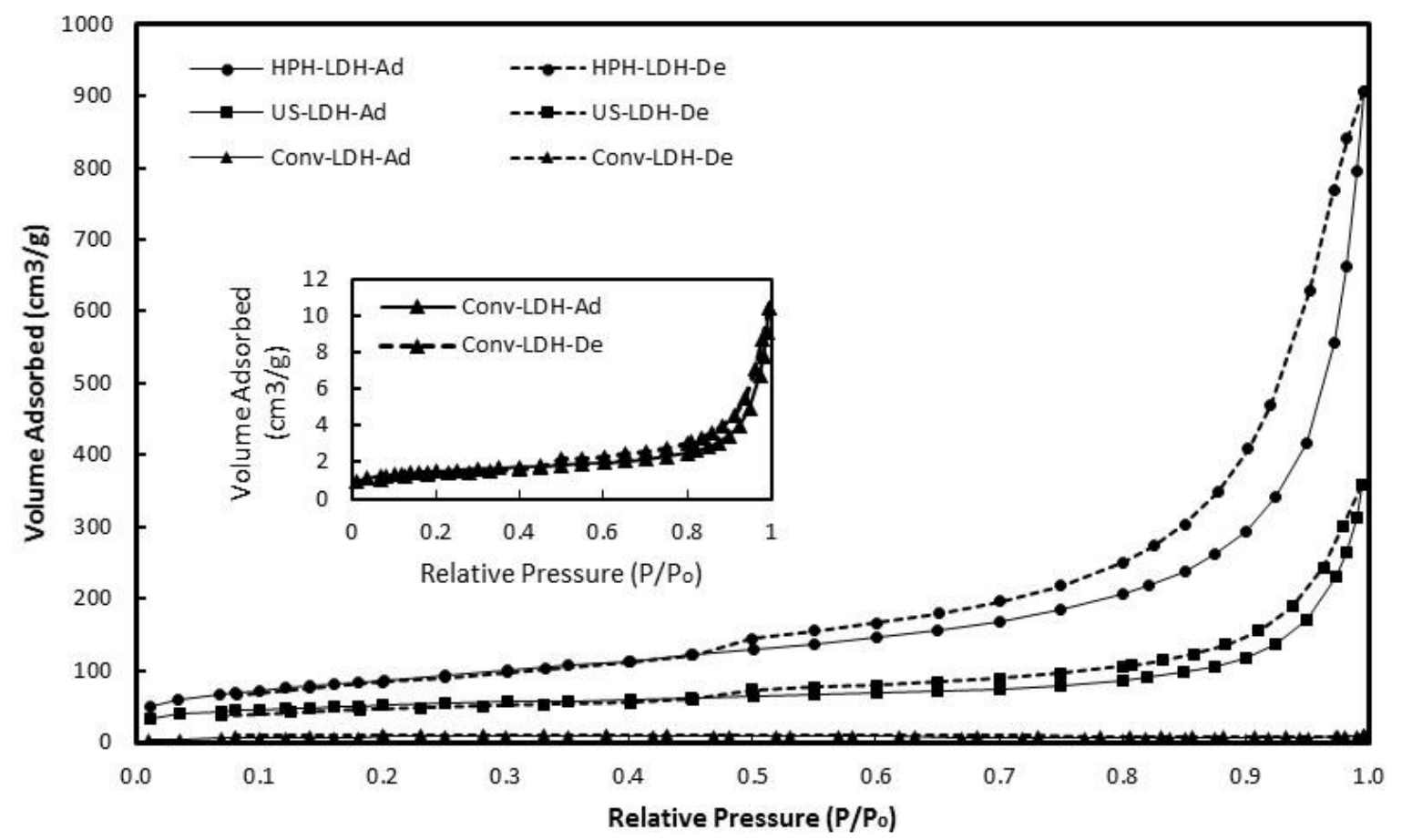

Figure 5: Nitrogen adsorption-desorption isotherms for Conv-LDH, US-LDH and HPH-LDH samples (inserted image shows the enlarged isotherm for Conv-LDH) 
Table 3: Surface content and binding energy for $N 1$ s and $O$ 1s levels for LDH, US-LDH and HPH-LDH

\begin{tabular}{|c|c|c|c|c|c|c|c|c|c|c|c|}
\hline \multirow[b]{3}{*}{$\begin{array}{l}\text { Preparation } \\
\text { Route }\end{array}$} & \multirow[b]{3}{*}{ Sample } & \multicolumn{5}{|c|}{ N 1s Spectra } & \multicolumn{5}{|c|}{ O 1s Spectra } \\
\hline & & \multicolumn{2}{|c|}{$\begin{array}{c}\text { Binding Energy } \\
(\mathrm{eV})\end{array}$} & \multicolumn{2}{|c|}{$\begin{array}{c}\text { Area } \\
(\text { cps eV })\end{array}$} & \multirow{2}{*}{$\begin{array}{c}\text { Content } \\
\left(\frac{\text { Free }}{\text { Protonated }}\right)\end{array}$} & \multicolumn{2}{|c|}{$\begin{array}{c}\text { Binding Energy } \\
(\mathrm{eV})\end{array}$} & \multicolumn{2}{|c|}{$\begin{array}{c}\text { Area } \\
(\text { cps eV) }\end{array}$} & \multirow{2}{*}{$\begin{array}{l}\text { Content } \\
\left(\frac{O H^{-}}{O^{2-}}\right)\end{array}$} \\
\hline & & $\begin{array}{l}\text { Free } \\
\text { Amine }\end{array}$ & $\begin{array}{l}\text { Protonated } \\
\text { Amine }\end{array}$ & $\begin{array}{l}\text { Free } \\
\text { Amine }\end{array}$ & $\begin{array}{l}\text { Protonated } \\
\text { Amine }\end{array}$ & & $\begin{array}{c}\text { Lattice } \\
\text { Oxygen } \\
\left(\mathrm{O}^{2-}\right)\end{array}$ & $\begin{array}{c}\text { Hydroxyl } \\
\text { Group } \\
\left(\mathrm{OH}^{-}\right)\end{array}$ & $\begin{array}{c}\text { Lattice } \\
\text { Oxygen } \\
\left(\mathrm{O}^{2-}\right)\end{array}$ & $\begin{array}{c}\text { Hydroxyl } \\
\text { Group } \\
\left(\mathrm{OH}^{-}\right)\end{array}$ & \\
\hline Conventional & Conv-LDH & 396.7 & 400.8 & 640.0 & 626.4 & 1.02 & 529.9 & 531.4 & 7633.2 & 1011.8 & 0.13 \\
\hline Ultrasonic & US-LDH & 396.9 & 400.3 & 574.1 & 876.6 & 0.65 & 529.8 & 531.3 & 10109.2 & 2053.1 & 0.21 \\
\hline Hydrothermal & HPH-LDH & 395.4 & 399.1 & 349.7 & 791.2 & 0.44 & 530.4 & - & 60510.2 & - & - \\
\hline
\end{tabular}

Table 4: EDX elemental analysis and CO2 uptake of prepared LDH and calculation results for the molecular formulas, removed SDS and effective amine efficiency

\begin{tabular}{|c|c|c|c|c|c|c|c|c|c|c|}
\hline Sample & $\begin{array}{c}\mathrm{N} \\
(\mathrm{wt} \%)\end{array}$ & $\begin{array}{c}C \\
(w t \%)\end{array}$ & $\begin{array}{c}\mathrm{S} \\
(\mathrm{wt} \%)\end{array}$ & $\begin{array}{l}\text { Molecular formula } \\
\qquad(\mathrm{mmol} / \mathrm{g})^{\mathrm{a}}\end{array}$ & SDS/APTS & $\begin{array}{c}\text { Amine } \\
\text { Loading } \\
(\mathrm{mmol} / \mathrm{g})\end{array}$ & $\begin{array}{c}\mathrm{CO}_{2} \\
\text { Adsorbed } \\
(\mathrm{mmol} / \mathrm{g})\end{array}$ & $\begin{array}{c}\text { SDS } \\
\text { removed } \\
(\%)\end{array}$ & $\begin{array}{c}\text { Effective } \\
\text { amine } \\
\text { loading } \\
(\mathrm{mmol} / \mathrm{g})^{\mathbf{b}}\end{array}$ & $\begin{array}{c}\text { Effective } \\
\text { Amine } \\
\text { Efficiency }\end{array}$ \\
\hline Conv-LDH & 0.65 & 51.35 & 11.41 & {$\left[\mathrm{C}_{12} \mathrm{H}_{25} \mathrm{SO}_{4}\right]_{3.57^{-} \cdot\left[\mathrm{C}_{0.01} \mathrm{H}_{0.52} \mathrm{SiNO}_{3}\right]_{0.46}}$} & 7.68 & 0.46 & 0.82 & & & \\
\hline Conv-MEA & 6.60 & 26.60 & 1.05 & {$\left[\mathrm{C}_{12} \mathrm{H}_{25} \mathrm{SO}_{4}\right]_{0.33^{-} .}\left[\mathrm{C}_{3.87} \mathrm{H}_{10.17} \mathrm{SiNO}_{3}\right]_{4.71}$} & 0.07 & 4.71 & 1.45 & 99.09 & 4.25 & 0.15 \\
\hline US-LDH & 3.11 & 51.16 & 11.08 & {$\left[\mathrm{C}_{12} \mathrm{H}_{25} \mathrm{SO}_{4}\right]_{3.46} \cdot\left[\mathrm{C}_{0.49} \mathrm{H}_{1.72} \mathrm{SiNO}_{3}\right]_{2.22}$} & 1.56 & 2.22 & 0.66 & & & \\
\hline US-MEA & 7.34 & 30.64 & 0.60 & {$\left[\mathrm{C}_{12} \mathrm{H}_{25} \mathrm{SO}_{4}\right]_{0.19^{-} .}\left[\mathrm{C}_{4.44} \mathrm{H}_{11.60} \mathrm{SiNO}_{3}\right]_{5.24}$} & 0.04 & 5.24 & 1.37 & 97.71 & 3.02 & 0.24 \\
\hline HPH-LDH & 2.55 & 13.68 & 1.50 & {$\left[\mathrm{C}_{12} \mathrm{H}_{25} \mathrm{SO}_{4}\right]_{0.47^{-}} \cdot\left[\mathrm{C}_{3.17} \mathrm{H}_{8.43} \mathrm{SiNO}_{3}\right]_{1.82}$} & 0.26 & 1.82 & 0.88 & & & \\
\hline HPH-MEA & 7.36 & 9.27 & 0.82 & {$\left[\mathrm{C}_{12} \mathrm{H}_{25} \mathrm{SO}_{4}\right]_{0.26}-\left[\mathrm{C}_{0.88} \mathrm{H}_{2.71} \mathrm{SiNO}_{3}\right]_{5.26}$} & 0.05 & 5.26 & 3.75 & 81.06 & 3.44 & 0.83 \\
\hline
\end{tabular}

a. All Nitrogen, Carbon and Sulphur elements in the grafted LDHs were attributed to come from organic compounds used for intercalation

b. Effective amine loading = Difference in amine loading before and after LDH modification

c. Effective Amine Efficiency = Difference in $\mathrm{CO}_{2}$ adsorbed before and after LDH modification/effective amine loading 
Table 5: $\mathrm{CO}_{2}$ kinetic model parameters, $R^{2}$ and standard errors (\%) for prepared $L D H s$ and amine functionalized $L D H s$ at $55^{\circ} \mathrm{C}$ and $1 \mathrm{~atm}$

\begin{tabular}{|c|c|c|c|c|c|c|c|c|c|c|c|c|c|c|c|}
\hline \multirow{2}{*}{ Samples } & \multicolumn{2}{|c|}{$\begin{array}{c}\text { Pseudo 1st } \\
\text { Order }\end{array}$} & \multirow{2}{*}{$\begin{array}{l}\text { Err } \\
(\%)\end{array}$} & \multirow[t]{2}{*}{$\mathbf{R}^{2}$} & \multicolumn{2}{|c|}{$\begin{array}{l}\text { Pseudo 2nd } \\
\text { Order }\end{array}$} & \multirow{2}{*}{$\begin{array}{l}\text { Err } \\
(\%)\end{array}$} & \multirow[t]{2}{*}{$\mathbf{R}^{2}$} & \multicolumn{5}{|c|}{ Double Exponential } & \multirow{2}{*}{$\begin{array}{l}\text { Err } \\
(\%)\end{array}$} & \multirow[t]{2}{*}{$\mathbf{R}^{2}$} \\
\hline & $\overline{\mathbf{x}_{\mathrm{e}}}$ & $\overline{\mathbf{k}_{\mathbf{f}}}$ & & & $\overline{\mathbf{x}_{\mathrm{e}}}$ & $\overline{\mathbf{k}_{\mathrm{s}}}$ & & & $\overline{\mathbf{x}_{\mathrm{e}}}$ & $\overline{A_{1}}$ & $\overline{\mathbf{A}_{2}}$ & $\mathbf{k}_{1}$ & $\mathbf{k}_{\mathbf{2}}$ & & \\
\hline Conv-LDH & 0.75 & 0.12 & 0.07 & 0.9766 & 0.83 & 0.23 & 0.34 & 0.9483 & 0.76 & 0.31 & 0.55 & 0.31 & 0.10 & 3.71 & 0.9815 \\
\hline US-LDH & 0.55 & 0.09 & 0.37 & 0.9062 & 0.63 & 0.20 & 0.81 & 0.9631 & 0.85 & 0.47 & 0.45 & 0.01 & 0.23 & 9.24 & 0.9897 \\
\hline HPH-LDH & 0.89 & 0.05 & 0.14 & 0.9356 & 1.14 & 0.04 & 0.38 & 0.9034 & 0.88 & 0.48 & 0.48 & 0.06 & 0.06 & 0.28 & 0.9421 \\
\hline Conv-MEA & 1.33 & 0.13 & 0.10 & 0.8786 & 1.46 & 0.14 & 0.08 & 0.9711 & 1.41 & 0.75 & 0.67 & 0.05 & 0.69 & 0.41 & 0.9968 \\
\hline US-MEA & 1.32 & 0.05 & 0.10 & 0.9826 & 1.67 & 0.03 & 0.28 & 0.9709 & 1.30 & 0.70 & 0.70 & 0.06 & 0.06 & 0.09 & 0.9861 \\
\hline НРН-MEA & 3.52 & 0.35 & 2.06 & 0.8733 & 3.72 & 0.17 & 2.34 & 0.9155 & 3.58 & 1.63 & 2.04 & 0.14 & 1.07 & 2.01 & 0.9327 \\
\hline
\end{tabular}


Figure 5 shows the $\mathrm{N}_{2}$ adsorption-desorption isotherms for Conv-LDH, US-LDH and HPH-LDH samples. The adsorbents show a type IV isotherm with H1 hysteresis loop characterized with the formation of a monolayer accompanied by multilayers occurring as a result of capillary condensation. This is a characteristic feature of mesoporous materials [24-26] validating the data represented in Table 1. These H1-hysteresis type materials are attributed to the formation of aggregates or compact uniform spheres as shown in the SEM images [26]. The hysteresis loop for the HPH-LDH was observed to be broader than that of US- and Conv-LDHs indicating the extent of capillary condensation in the samples. It is observed that the volume adsorbed by HPH-LDH is 3 folds that of US-LDH, which in turn is $\sim 30$ folds of the conventional LDH. This depicts an enhancement in the pore structure of the samples and is attributed to the enhancement of nucleation and precipitate growth resulting from improved dissolution and reaction process via sonication [20] and hydrothermal crystallization [27].
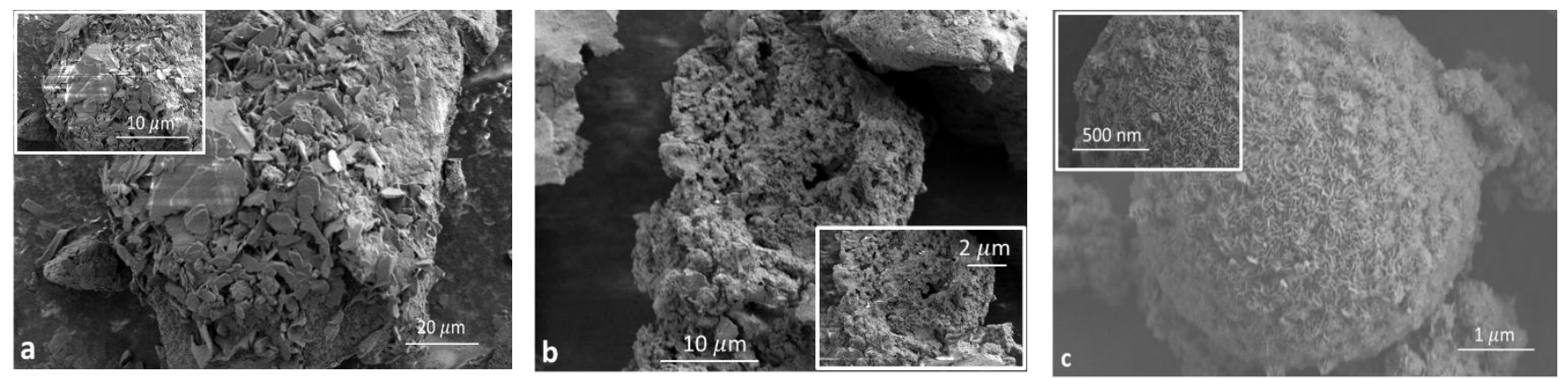

Figure 6: SEM images of prepared LDHs (a) Conv-LDH, (b) US-LDH, and (c) HPH-LDH

Considering the surface texture of the samples, Figure 6 shows images of surface morphology of the prepared LDH samples. It can be seen from the figure that Conv-LDH presents a bouldershaped material with aggregated flaky plate-like surface. This may be explained by the formation of shell-core structure caused by the sequential reduction of two different metallic ions [10, 28] resulted from the difference in the reduction potentials of $\mathrm{Al}^{3+}$ and $\mathrm{Mg}^{2+}$ ions. This is similar to the sonication route sample but without the plate-like surface. Surface of these samples displayed more pores attributable to high-speed jets or shock waves resulting from bubble collapse [28]. Unlike the conventionally and ultrasonically prepared samples, the hydrothermal LDHs were smaller and of spherical structure, hence increasing the effective surface area for adsorption. 


\subsection{Impact of preparation route on surface chemical content}

To investigate the impact of preparation route on the amine functional group and extent of oxidation on the surface of the adsorbent, XPS analysis was conducted. The N 1s and O 1s spectra are shown in Figure 7 and the data are summarized in Table 3. The deconvolution of $\mathrm{N}$ 1s spectra has two peaks at $c a .396( \pm 0.9) \mathrm{eV}$ and $400( \pm 0.9) \mathrm{eV}$ respectively (Figure 7a). These are assigned to free amine and protonated amines respectively [10, 29]. The concentration of free amino group decreased for species prepared via ultrasonic and hydrothermal mediation. This reveals that despite the enhanced physical properties of the adsorbent, ultrasonic and hydrothermal process has a reduced amount of free amino active sites on the adsorbent. This can be explained by the enhanced protonation of the free amine groups. However, the amine loading (deduced by the total concentration of $\mathrm{N}$ atom) is highest in species prepared via ultrasonic irradiation and least in the hydrothermal process. This can be partially attributed to the efficient ejection of hydroxyl radicals from bubbles during cavitation, which readily hydrates the anions; hence bonding the anions within the interlayers of the adsorbent [30]. This can be supported by the high content of hydroxyl group $\left(\mathrm{OH}^{-}\right)$in samples synthesised via ultrasonic irradiation. Similar to the N 1s spectra, Figure $7 \mathrm{~b}$ shows the deconvolution of the $\mathrm{O} 1 \mathrm{~s}$ spectra into two bands at $529.1 \mathrm{eV}$ and $531.6 \mathrm{eV}$ respectively. However, only the former band (with a $0.75 \mathrm{eV}$ chemical shift) was observed in the hydrothermally prepared sample. The first band relates to lattice oxygen $\left(\mathrm{O}^{2-}\right)$, while the latter relates to adsorbed surface oxygen or hydroxyl groups $\left(\mathrm{OH}^{-}\right)[13,31]$. The results summarised in Table 3 show that the ratio of hydroxyl group to lattice oxygen increased with ultrasonic irradiation but decreased with hydrothermal synthesis. This indicates that the surface concentration of the $\mathrm{OH}^{-}$group is least for the hydrothermal process and greatest in the ultrasonic mediated process. This is consistent with findings from $\mathrm{CO}_{2}$-TPD analysis. This can be ascribed to the boosted crystallization of the metal oxides due to enhanced transport properties of supercritical water, a characteristic of the hydrothermal process [32]. Supplementary documents (Tables S1 and S2) support this finding. This hypothesis is made visible with the aid of the EDX spectroscopy (see supplementary documents, Figure S1), which shows the enhanced dispersion of the metals $\left(\mathrm{Al}^{3+}\right.$ and $\mathrm{Mg}^{2+}$ ) across the adsorbent. The high concentration of lattice oxygen in the hydrothermal route can be associated to the increased $\mathrm{Mg}$ content in the form of $\mathrm{Mg}-\mathrm{O}-\mathrm{Mg}$ groups or $\mathrm{Mg}^{2+}-\mathrm{O}^{2-}$ ion pairs [33]. 
$\mathrm{CO}_{2}$-TPD analysis was used to measure the surface basicity of the synthesised LDHs (Figure 6). Surface basicity increases with increase in desorption temperature [6]. As shown in Figure 6a, the desorption of $\mathrm{CO}_{2}$ basically occurs at overlapping peaks of $270{ }^{\circ} \mathrm{C}(\alpha)$ and $363{ }^{\circ} \mathrm{C}(\beta), 474{ }^{\circ} \mathrm{C}(\gamma)$ and $569^{\circ} \mathrm{C}(\delta)$. At low temperatures $\left(<400{ }^{\circ} \mathrm{C}\right)$, desorption of $\mathrm{CO}_{2}$ is related to released $\mathrm{CO}_{2}$ from bicarbonates formed on weak and medium basic sites [10]. These weak sites are characteristics of the $\mathrm{Br} ø$ nsted $\mathrm{OH}^{-}$group which are observed to be more prominent in the conventional and ultrasonic mediated routes [6] (Figure 6b). This corresponds with reported literatures validating that more weak and moderate basic sites are formed with the aid of ultrasonic irradiation [13]. Nonetheless, HPH-LDH desorbed $\mathrm{CO}_{2}$ at intermediate/high temperature states $\left(>400{ }^{\circ} \mathrm{C}\right)$. These are strong basic sites and relate to bi-dentate and mono-dentate carbonates adsorbed on low coordinative unsaturated lattice oxygen $\left(\mathrm{O}^{2-}\right)$. This tends to agree with XPS and EDX results. A practical application of the basicity of this material vary from the recovery of $\mathrm{CO}_{2}$ from flue gases at low basic strength (low temperature level) to higher basic strength (high temperature level) processes like sorption enhanced hydrogenation. The latter has the advantage of ease of regeneration at low temperature gradients [6]. 


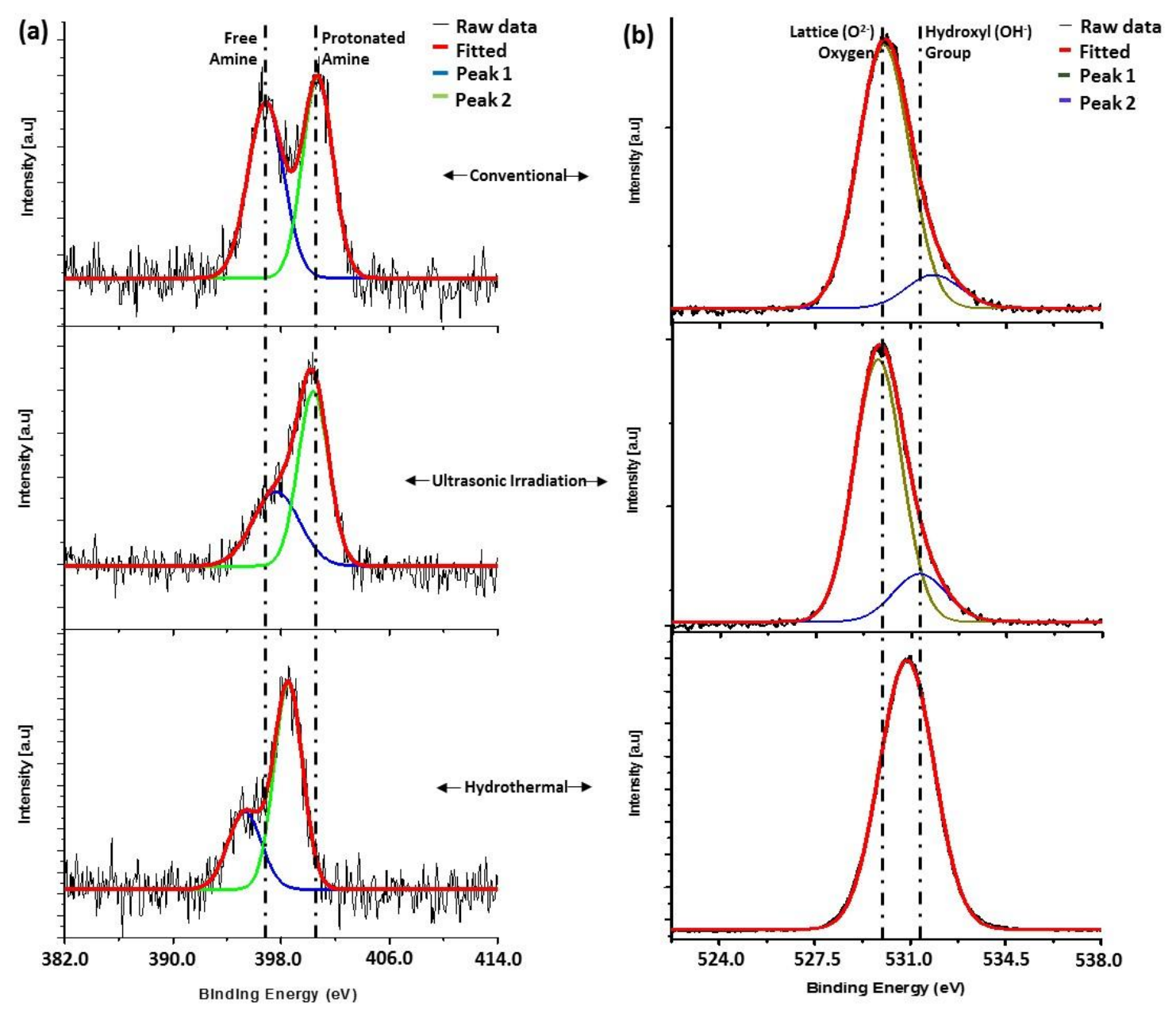

Figure 7: XPS spectra of conventionally-, ultrasonically- and hydrothermally-prepared LDHs for (a) N 1s and (b) O 1s.
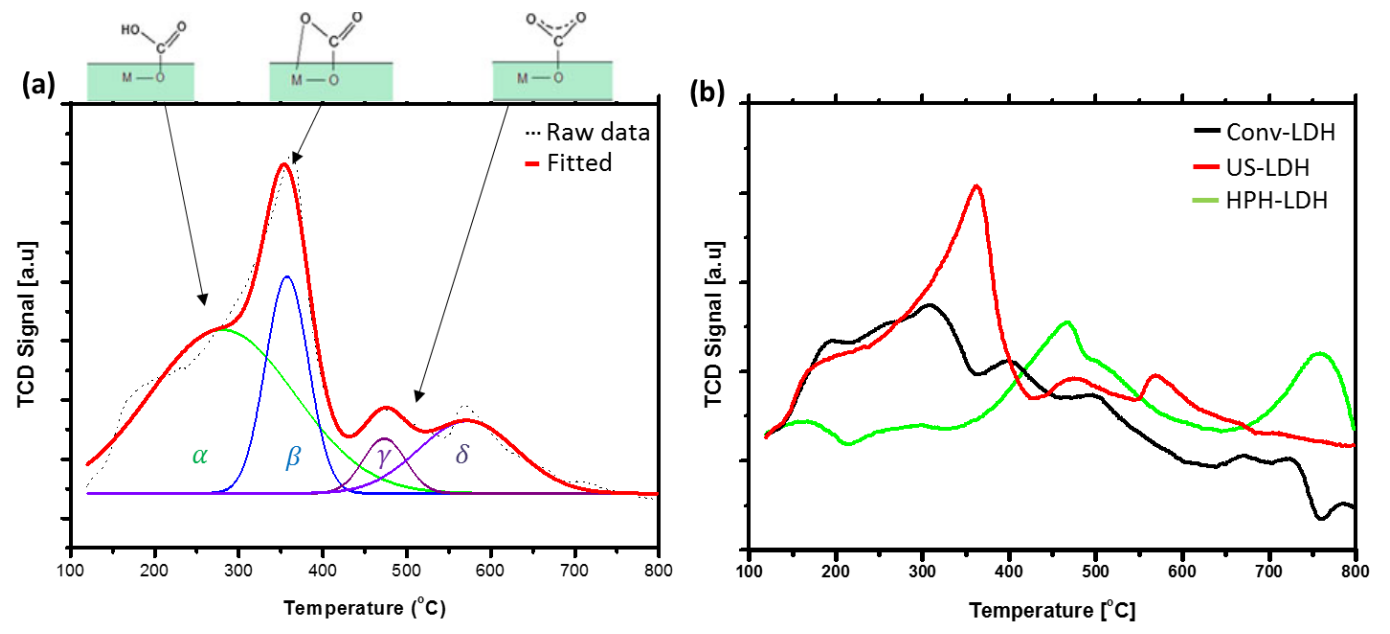

Figure 6: $\mathrm{CO}_{2}$-TPD of synthesised LDH (a) Deconvolution of the $\mathrm{CO}_{2}$-TPD of UH-LDH and (b) Comparison of $\mathrm{CO}_{2}$-TPD of Conv-LDH, US-LDH and HPH-LDH. 
To further understand the surface chemical content of the adsorbent, the amino functional group necessary for adsorption of $\mathrm{CO}_{2}$ was quantified using EDX results (See supplementary data Table S1). Table 4 shows the elemental analysis of each prepared sample using the EDX results from Table S1. As shown in Table 4, the amine loading was more effective using ultrasonic mediated route with $2.22 \mathrm{mmol} / \mathrm{g}$ as against 1.82 and $0.46 \mathrm{mmol} / \mathrm{g}$ for ultrasonic-assisted hydrothermal and the conventional route respectively. However, after functionalization of the adsorbent, the amine loading increased to $4.71,5.24$ and $5.26 \mathrm{mmol} / \mathrm{g}$ for conventional, ultrasonic and ultrasonicassisted hydrothermal synthesis respectively. This is observed to be a significant improvement compared to those results reported in literatures as documented in supplementary Table S3.

\subsection{Impact of preparation route on structural configuration}

Figure 7 shows the XRD analysis of synthesised LDHs. Typical Mg-Al LDH exhibits three separate peak regions: (a) high angle region $\left(2 \theta>60^{\circ}\right)$ consisting of $h k 0$ and $h k l$ reflections attributed to the ordering within the metal hydroxide layers, (b) the mid-angle region $\left(30^{\circ}<2 \theta<\right.$ $60^{\circ}$ ) attributed to non-basal reflection $0 \mathrm{kl}$ whose locations are characteristic of the structural disorder and, (c) the low angle region $\left(2 \theta<30^{\circ}\right)$ consisting of the $00 l$ and $h 00$ basal reflections characteristic of the size of intercalated anion [34]. These XRD reflections have been indexed according to the hexagonal lattice with rhombohedral symmetry (space group: R3m) [34-36]. A brief look from the figure indicates that the intensity of all reflections increased with the hydrothermal process. This is associated with an increase in interlayer water molecule and partial hydroxylation of double hydroxide sheets [37]. For the high angle region, peak at $2 \theta=60^{\circ}$ containing overlapping reflections of (110) and (113) attributed to interlayer spacing indicates that the hydrothermal route increases interlayer spacing. This is reported to be associated with the presence of oxygen-containing functional groups [38], which is observed to be highest in the hydrothermal synthetic method due to high metallic deposition and dispersion. Hydrothermal route is considered an important synthetic method for the preparation of highly dispersed heterogeneous materials. This is due to its tendency to dissolve and recrystallize materials at high temperature and pressure [39]. The 200 lattice peak reflection explains the crystal size of the material and its intensity is dependent on chemical composition and molar $\left(\mathrm{M}^{2+} / \mathrm{M}^{3+}\right)$ ratio [40]. Consequently, the strength of basic sites should vary with the peak intensity, thus validating the effect of deposition 
and dispersion of divalent and trivalent cations on basic strength. For the low and mid-angle region, the increase in peak intensities can be attributed to change in configuration of the interlayer nitrate from single flat-lying molecular layer to multiple layers and with different orientation [35, 41]. To this effect, using the results of X-ray diffraction and polarized attenuated total reflection FTIR spectroscopy, Wang and Wang [41] explained that the change in orientation of interlayer nitrate anions is dependent on chemical composition of LDH. This corresponds with XPS and TPD results.

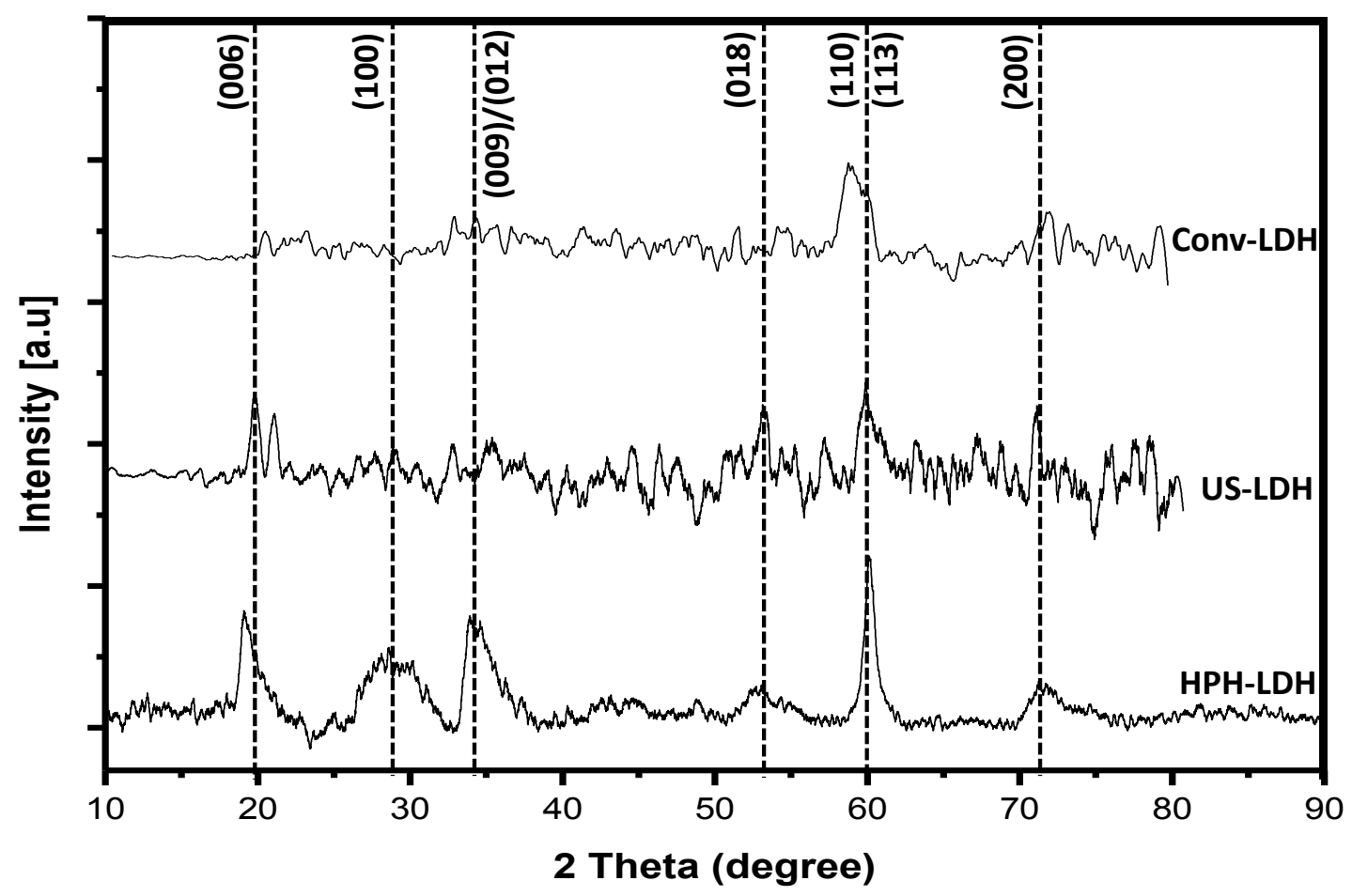

Figure 7: X-ray diffraction patterns of synthesised Mg/Al LDHs via conventional co-precipitation (Conv-LDH), ultrasonic irradiation (US-LDH) and sonochemical-assisted hydrothermal route (HPH-LPH).

\subsection{Impact of preparation route on thermal stability}

The thermal stability of the prepared samples were obtained using Netzsch STA 449 F3 TGA from $25{ }^{\circ} \mathrm{C}$ to $1000{ }^{\circ} \mathrm{C}$ with a $10{ }^{\circ} \mathrm{C} / \mathrm{min}$ ramp. Figure 8 shows the TGA profiles for thermal disintegration of the prepared samples. Three distinct temperature phases were observed for material disintegration: $\mathrm{T}<\sim 150{ }^{\circ} \mathrm{C}, \sim 150<\mathrm{T}<\sim 750{ }^{\circ} \mathrm{C}$ and $\sim 750{ }^{\circ} \mathrm{C}<\mathrm{T}$. The first phase of weight loss $\left(\mathrm{T}<\sim 150{ }^{\circ} \mathrm{C}\right)$ is attributed to the loss of interstitial water molecules. While for the second 
phase $\left(\sim 150<\mathrm{T}<\sim 750{ }^{\circ} \mathrm{C}\right)$, the decomposition can be ascribed to the dehydroxylation and breakdown of the organic alkyl chain of the LDH. The observed irregular decomposition curve in this stage may indicate the occurrence of an uneven bonding structure, resulting to multi-stage dehydroxylation processes. The final weight loss $\left(\sim 750{ }^{\circ} \mathrm{C}<\mathrm{T}\right)$ results from the decomposition of the sulphate species residuum [10]. From Figure 8a-c, it can be inferred that the thermal strength of HPH-LDH is higher than that of the conventional and ultrasonically prepared samples with a total weight loss of 68.98 amd $68.12 \%$ respectively, when compared to $10.56 \%$ of HPH-LDH as tabulated in Table S4 (supplementary document). For the first phase, the moisture content decreased significantly from 8.35 to $3.41 \%$ as the preparation process changed from conventional to hydrothermal. A more drastic reduction was experienced with the interstitial moisture content (second phase). This can be attributed to the enhanced bonding introduced by the hydrothermal process forming multi-layers as estimated from the XRD results. The third degradation phase infers that ultrasonic process is more likely to deposit more sulphate species, while the hydrothermal process is the least to do so. This is corresponds to the results obtained from XPS analysis (Table S2, supporting document). This indicates that the adoption of the hydrothermalroute may be beneficial to the synthesis of a more stable material than the ultrasonic and conventional method.

After introducing amine modification of the samples, the decomposition curves clearly show different behaviour compared with that of the untreated LDHs prepared by the same method. The curves display a well-defined three phase decomposition steps unlike the untreated ones, as can be seen from Figure 8(d-f). Within the same temperature range as that of the LDHs, the MEA-treated LDHs showed higher moisture content than the unprocessed ones except for the hydrothermal samples. This can be caused by the presence of leftover MEA molecules after the extraction process, which may have been strongly bonded to the hydrothermal samples resulting to the increase in weight loss in the second and third phase. However, for the ultrasonic and conventional route, the weight losses in the second and third phase reduced significantly, benefitting to production of a more stable material than the pure LDH. This can be explained by the reduced presence of the surfactant after the amine extraction. Showing from the total weight loss, it can be concluded that the thermal strength of the hydrothermal samples (total weight loss: $38.17 \%$ ) even 
after amine extraction is better than that of the conventional and ultrasonically prepared samples (total weight loss: 52-53\%).
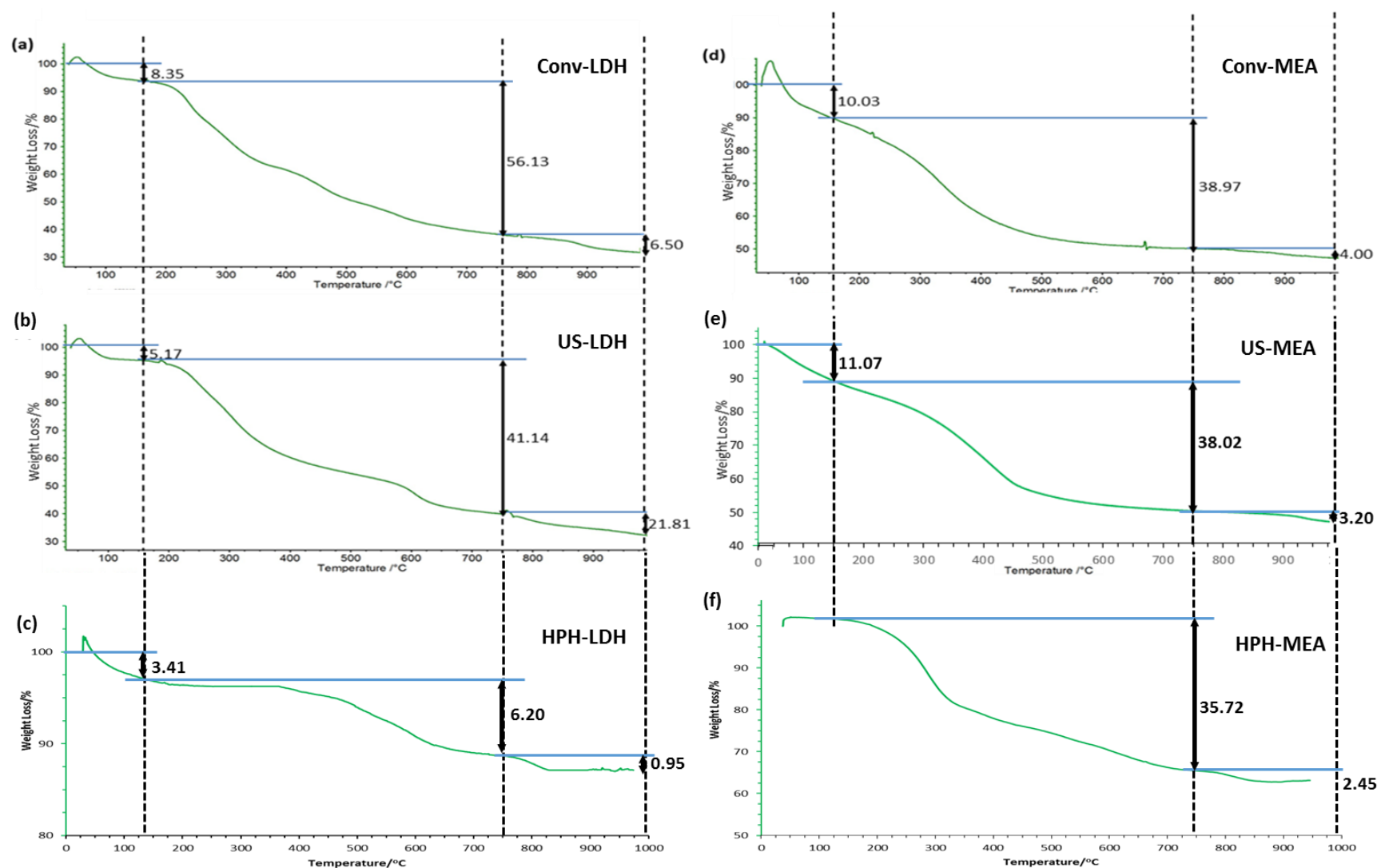

Figure 8: TGA curves comparing thermal stabilities of LDHs prepared via conventional and ultrasonic irradiation: (a) Conv-LDH, (b) UH-LDH and (c) HPH-LDH; as well as with amine modified LDHs: (d) Conv-MEA (e) UH-MEA and (f) HPH-MEA.

\subsection{Impact of preparation route on effective amine efficiency, $\mathrm{CO}_{2}$ uptake, regeneration efficiency and cyclic efficiency}

The average $\mathrm{CO}_{2}$ uptake for the various synthesis route is shown in Figure 9. The results show a reduction in $\mathrm{CO}_{2}$ uptake for the US-LDH when compared to the conventional, Conv-LDH. This corresponds to results from previous study [10]. However, an insignificant increase in $\mathrm{CO}_{2}$ uptake was observed for the hydrothermally prepared sample, HPH-LDH. Despite the enhanced textural properties of HPH-LDH, the adsorption capacity of this adsorbent showed no appreciable improvement. However, after amine extraction, the amine loading of adsorbents increased for all 
considered routes. This is part due to the exfoliation of the surfactant (SDS) (shown in Table 4) which varied from ca. 99\%, 98\% and $81 \%$ in the conventional, ultrasonic and hydrothermal processes respectively. As a result, this impacted on the effective amine loading and efficiency of the adsorbent. The effective amine loading was computed as the difference in amine loading before and after amine extraction, whereas effective amine efficiency was computed as the ratio of the difference in $\mathrm{CO}_{2}$ adsorbed before and after amine extraction to effective amine loading. HPHMEA showed the highest effective amine efficiency of 0.83 compared to 0.24 and 0.15 for USMEA and Conv-MEA respectively. This can be partly related to the improved deprotonation of protonated amino groups resulting from enhanced transport properties, characteristic of the hydrothermal process [32]. This expounds the impact of sonication and hydrothermal pretreatment in deprotonating protonated and/or probable dispersion of amino sites during LDHamine modification. Consequently, the adsorption capacity of HPH-LDH increases from 0.88-3.75 $\mathrm{mmol} / \mathrm{g}$ when compared to the increase of Conv-LDH from $0.82-1.45 \mathrm{mmol} / \mathrm{g}$ (supplementary document, Table S5). This indicates that $\mathrm{CO}_{2}$ uptake was stimulated more by chemisorption rather than physisorption. This can be attributed to the difference in basic strength of the prepared adsorbents.

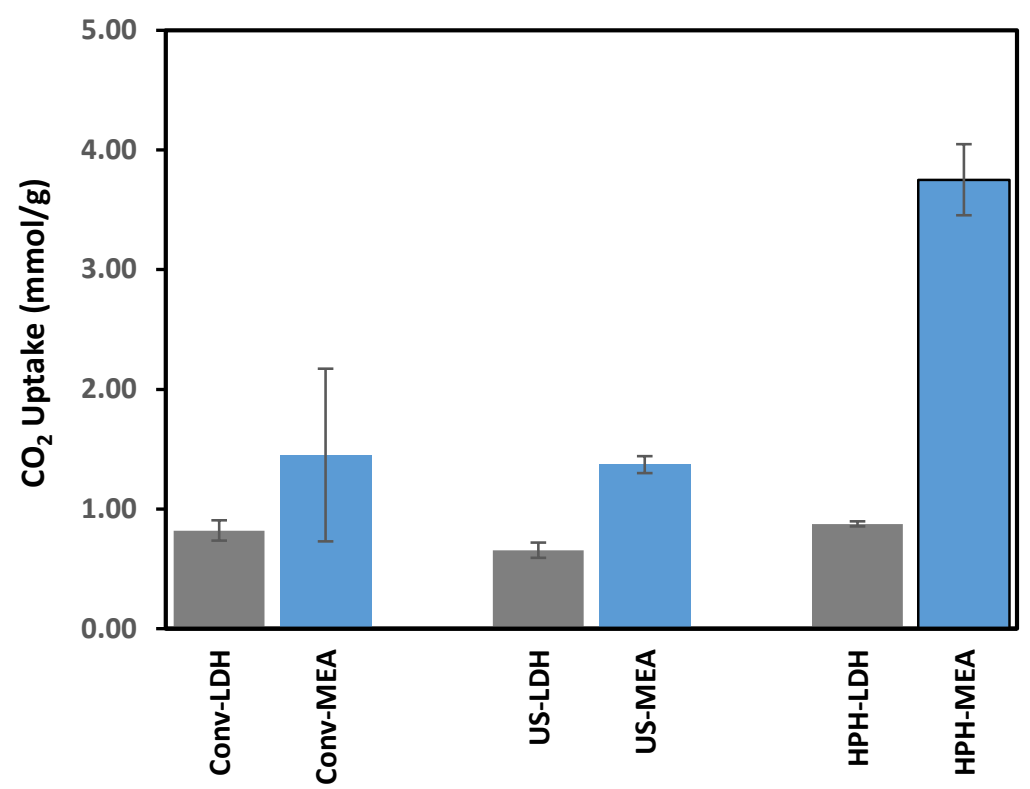

Figure 9: Average $\mathrm{CO}_{2}$ Uptake of $\mathrm{LDH}$ and amine-modified $\mathrm{LDH}$ prepared via the different routes.

Thermal swing regeneration in an atmosphere of $\mathrm{N}_{2}$ was adopted for the regeneration of the amine modified adsorbent. A typical example of the regeneration profile is shown in Figure S2 of 
supplementary document. In this figure, the effect of the preparation route on the cyclic sorption capacity of the adsorbent is presented. Amount of $\mathrm{CO}_{2}$ desorbed was measured after the first adsorption cycle prior to the second adsorption process. Consecutively, the regeneration and cyclic efficiencies of the adsorbents were calculated (supplementary document, Table S5). From the tabulated results in the figure, it is observed that HPH-MEA has the highest regeneration efficiency of $93 \%$. This is a significant change when compared to $78 \%$ and $86 \%$ regeneration efficiencies for US-MEA and Conv-MEA respectively. However, the cyclic efficiency (computed as the percentage ratio of $\mathrm{CO}_{2}$ uptake from $2^{\text {nd }}$ cycle to $1^{\text {st }}$ cycle) of US-MEA is $76 \%$ compared to $60 \%$ and $53 \%$ for Conv-MEA and HPH-MEA respectively. Analysing the cyclic and regenerated efficiencies, it can be projected that the ultrasonic mediated synthesis route has an effective adsorption potential than the other two processes. It is observed that of the $78 \%$ regenerated active site, approx. $76 \%$ of this active site underwent the $2^{\text {nd }}$ adsorption process. This is lesser in ConvMEA and HPH-MEA respectively. The degradation in the cyclic adsorption efficiencies of these samples can be ascribed to the secondary reactions occurring between the amino group and $\mathrm{CO}_{2}$ as elaborated in previous study [10]. This implies that the ultrasonic mediated route is more suitable for the synthesis of long term effective $\mathrm{CO}_{2} \mathrm{LDH}$ adsorbents. However, the hydrothermal process

provides a significant change in sorption capacity of the adsorbent. Optimization of these two processes will contribute immensely to both sorption efficiency and effectiveness.

\subsection{Comparison of kinetic models}

From simulation results, the experimental kinetic uptake data for the samples synthesized from the different synthesis routes are summarized in Table 5. Comparative plots of these data using the kinetic models discussed in the previous section are shown in Figure 102. The results show that the double exponential model produced the best fit for the experimental data (considering the $\mathrm{R}^{2}$ values). This unlike the pseudo first and second order model, is explained to account for the heterogeneity of the adsorbent's surface [16]. In other words, it describes the adsorption mechanism to comprise of two different adsorption sites. Consequently, elucidating the occurrence of more than one reaction mechanism [42]. Therefore, the double exponential model can be explained to be of best fit of the three models because it possibly accounts for both chemisorption and physisorption of $\mathrm{CO}_{2}$. Rate constants $\mathrm{k}_{\mathrm{i}=1,2}$ are the reaction rate coefficients for the respective adsorption mechanisms. The simulated results showed no particular variation of these parameter 
with the preparation route. The pre-exponential factors, $A_{i=1,2}$, indicate the weight coefficient that reflects the share of each adsorption mechanism [17]. From the tabulated results, it is observed that the second adsorption mechanism (indicated by $\mathrm{i}=2$ ) contributed a greater share towards $\mathrm{CO}_{2}$ adsorption in Conv-LDH. This gradually reduced in US-LDH and was approximately evenly distributed between the two mechanisms in HPH-LDH.
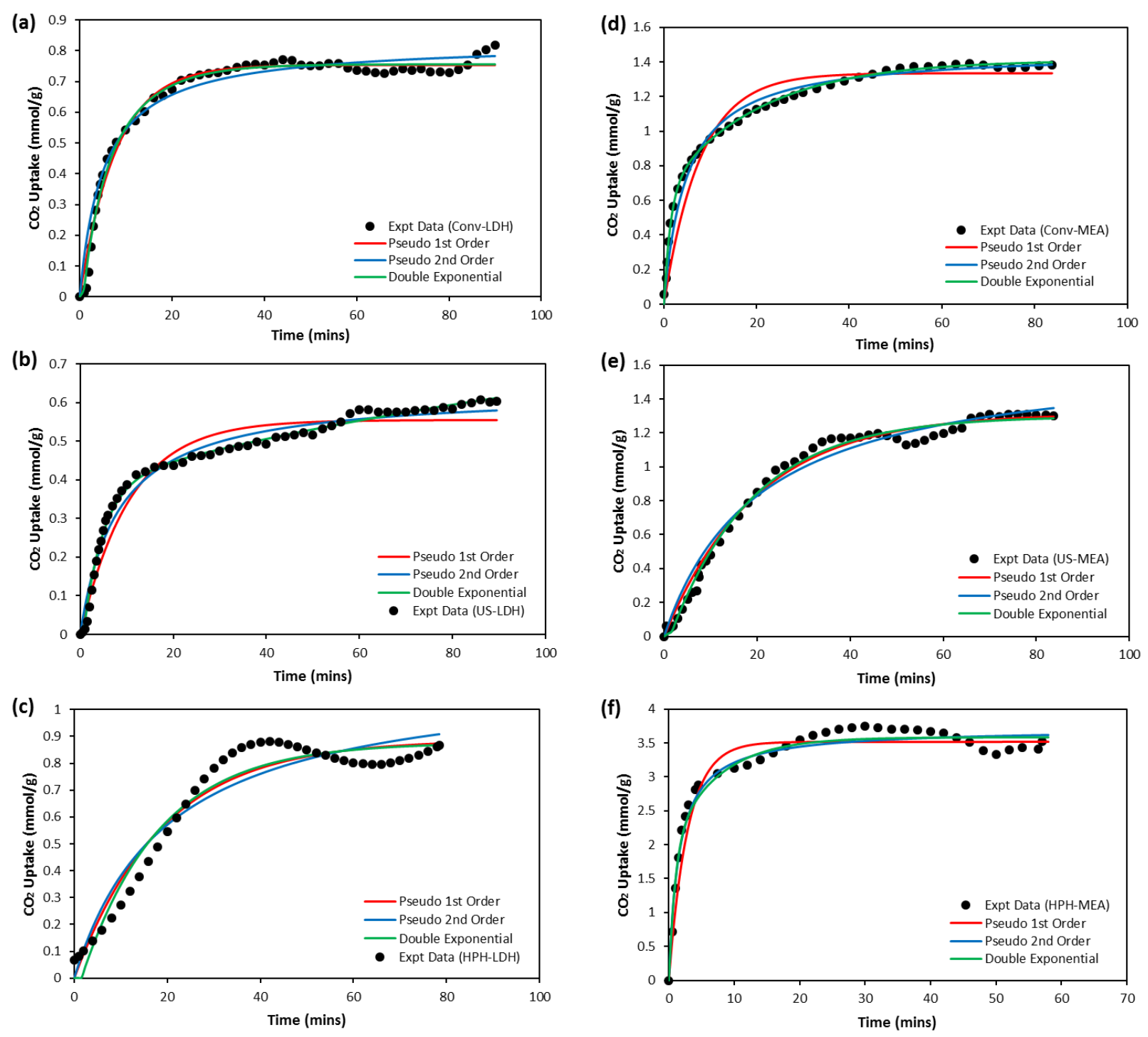

Figure 102: Comparison of different kinetic models with experimental data generated for adsorption kinetics of $\mathrm{CO}_{2}$ on (a) Conv-LDH, (b) US-LDH, (c) HPH-LDH, (d) Conv-MEA, (e) US-MEA and (f) HPH-MEA under and $55^{\circ} \mathrm{C}$ and $1 \mathrm{~atm}$. 
Pseudo first and second order model also provided a relatively good fit for the experimental results. However, comparing to pseudo second order model, pseudo first order provided a better fit for both Conv-LDH and HPH-LDH experimental data. This model better explains low surface coverage adsorption [43] whereas the pseudo second order model accounts for adsorption by chemisorption or adsorption at high adsorbate loadings [44]. Hence, it is logical to attribute the fitting of the second order model to US-LDH experimental data to the high amine loading. Considering the kinetic parameters, $\mathrm{k}_{\mathrm{f}}$ and $\mathrm{k}_{\mathrm{s}}$, which depicts the time scale for the process to reach equilibrium [17]. Inverse of these parameters indicates the time required for the process to attain equilibrium. As shown in Table 5, the $\mathrm{k}_{\mathrm{f}, \mathrm{s}}$ decreased in the following trend: conventional > ultrasonic > hydrothermal. This portrays that conventionally prepared LDH tends to attain equilibrium quicker than samples prepared from the other routes. Although pseudo first and second order models are associated with surface reaction control systems [17], the former was also derived by assuming film diffusion (FD) control [45] whereas the later simulates intra-particle diffusion (IPD)-driven kinetics of sorption for systems with both plane and spherical sorbent particles [46]. Consequently, it is likely to assume that IPD is more promoted in US-LDH.

After amine extraction, the double exponential model was also of best fit for the obtained experimental data. However, the reverse trend was observed for the share of each adsorption mechanisms. Summarised results (Table 5) show that both adsorption mechanism approximately contributed evenly in the adsorption process in Conv-MEA and US-MEA; but the second mechanism dominated in HPH-MEA. The nature of these mechanisms are undetermined in this study but can be seen to give an insight on the adsorption trend of each sample. Nonetheless, correlating these findings with characterization results, the first adsorption mechanism (indicated by $\mathrm{i}=1$ ) can be inferred to be associated with the physical textural properties of the adsorbent. Subsequently, the enhanced textural properties via ultrasonic and hydrothermal treatment resulted to the increase in the share of the first adsorption mechanism. However, after amine extraction, the second mechanism, assumed to be characterized by chemisorption, increased in share in the following trend: Conv-MEA < US-MEA < HPH-MEA. This can be attributed to the enhanced amine loading and effective amine efficiency. 


\section{CONCLUSION}

A detailed comparison study of the impact of ultrasonic and hydrothermal pre-treatment on the basic strength, textural characteristics, effective amine efficiency, $\mathrm{CO}_{2}$ adsorption and regeneration capacities of $\mathrm{Mg}-\mathrm{Al} \mathrm{LDH}$ has been conducted. In addition, the contribution of these preparation routes to the thermal stability and reaction kinetics was discussed. The results obtained using the kinetic models clearly indicate that prior to amine extraction, the adsorption of Mg-Al LDH adsorbent is mostly facilitated by physisorption while adsorption is mainly promoted by chemisorption after amine extraction. The conclusions reached as the result of the present study can be summarised:

(1) Characterization results show that the ultrasonic-assisted hydrothermal route immensely improves the porosity and surface morphology of the adsorbent. Furthermore, the surface chemical content was characterised with improved metal-ion distribution, hence increasing the basic strength of the adsorbent. This strongly contributes to the improved adsorption and regeneration capacities, as well as the thermal stability of the adsorbent.

(2) The reason that the ultrasonic-assisted hydrothermal treatment can effectively promote the adsorption capacity of the adsorbent can be partially attributed to the ascent in the amount of strong basic sites $\left(\mathrm{O}^{2-}\right)$ and subsequent decrease in both moderate (M-O) and weak $\left(\mathrm{OH}^{-}\right.$groups) basic sites resulting from the hydrothermal process.

(3) The cyclic adsorption efficiency of the sonochemical process is better than that of both hydrothermal and conventional preparation routes. The sonochemical route shows the potential for enhanced amine loading and cyclic efficiency, thus providing a more suitable and economical approach for synthesis of double layered hydroxides.

\section{ACKNOWLEDGEMENT}

This study was conducted at the International Doctoral Innovation Centre (IDIC). The authors would like to acknowledge the financial support from National Natural Science Foundation of China (NSFC) (Grant No. 21576141) and Zhejiang Provincial Natural Science Foundation (Grant 
No. LY15B060001) and Ningbo Key Research Project 'Absorption/catalytic technologies for the simultaneous removal of multi-pollutants from flue gas at power stations' (Grant No. 1012B10042), and from Ningbo Science and Technology Bureau, The University of Nottingham, Ningbo Education Bureau and China's MoST . The study is also partly supported by EPSRC (Grant no. EP/G037345/1). In addition,

\section{References}

[1] J. Wang, L.A. Stevens, T.C. Drage, C.E. Snape, J. Wood, Preparation and CO2 adsorption of amine modified layered double hydroxide via anionic surfactant-mediated route, Chemical Engineering Journal, 181-182 (2012) 267-275.

[2] J.D. Figueroa, T. Fout, S. Plasynski, H. McIlvried, R.D. Srivastava, Advances in CO2 capture technology-The U.S. Department of Energy's Carbon Sequestration Program, International Journal of Greenhouse Gas Control, 2 (2008) 9-20.

[3] M. Szabados, R. Mészáros, S. Erdei, Z. Kónya, Á. Kukovecz, P. Sipos, I. Pálinkó, Ultrasonically-enhanced mechanochemical synthesis of CaAl-layered double hydroxides intercalated by a variety of inorganic anions, Ultrasonics Sonochemistry, 31 (2016) 409-416.

[4] F. Cavani, F. Trifirò, A. Vaccari, Hydrotalcite-type anionic clays: Preparation, properties and applications, Catalysis Today, 11 (1991) 173-301.

[5] N.D. Hutson, B.C. Attwood, High temperature adsorption of $\mathrm{CO} 2$ on various hydrotalcite-like compounds, Adsorption, 14 (2008) 781-789.

[6] D.I. Ferrer, Supported Layered Double Hydroxides as CO2 Adsorbents for Sorption-enhanced H2 Production, Chemical Engineering, Imperial College, London, United Kingdom, Springer International Publishing Switzerland, 2016, pp. 209.

[7] L.H. Chagas, G.S.G. De Carvalho, W.R. Do Carmo, R.A.S. San Gil, S.S.X. Chiaro, A.A. Leitão, R. Diniz, L.A. De Sena, C.A. Achete, MgCoAl and NiCoAl LDHs synthesized by the hydrothermal urea hydrolysis method: Structural characterization and thermal decomposition, Materials Research Bulletin, 64 (2015) 207-215.

[8] Y. Gao, Z. Zhang, J. Wu, X. Yi, A. Zheng, A. Umar, D. O'Hare, Q. Wang, Comprehensive investigation of $\mathrm{CO} 2$ adsorption on $\mathrm{Mg}-\mathrm{Al}-\mathrm{CO} 3 \mathrm{LDH}$-derived mixed metal oxides, Journal of Materials Chemistry A, 1 (2013) 12782-12790.

[9] J.I. Di Cosimo, V.K. Díez, M. Xu, E. Iglesia, C.R. Apesteguía, Structure and Surface and Catalytic Properties of Mg-Al Basic Oxides, Journal of Catalysis, 178 (1998) 499-510.

[10] C.I. Ezeh, X. Huang, X. Yang, C.-g. Sun, J. Wang, Sonochemical surface functionalization of exfoliated LDH: Effect on textural properties, $\mathrm{CO} 2$ adsorption, cyclic regeneration capacities and 
subsequent gas uptake for simultaneous methanol synthesis, Ultrasonics Sonochemistry, 39 (2017) 330-343.

[11] D.P. Debecker, E.M. Gaigneaux, G. Busca, Exploring, tuning, and exploiting the basicity of hydrotalcites for applications in heterogeneous catalysis, Chemistry-A European Journal, 15 (2009) 3920-3935.

[12] Y. Mu, Y. Zhang, J. Fan, C. Guo, Effect of ultrasound pretreatment on the hydrothermal synthesis of SSZ-13 zeolite, Ultrasonics Sonochemistry, 38 (2017) 430-436.

[13] S. Zhao, H. Yi, X. Tang, F. Gao, Q. Yu, Y. Zhou, J. Wang, Y. Huang, Z. Yang, Enhancement effects of ultrasound assisted in the synthesis of NiAl hydrotalcite for carbonyl sulfide removal, Ultrasonics Sonochemistry, 32 (2016) 336-342.

[14] H.J. Jang, C.H. Lee, S. Kim, S.H. Kim, K.B. Lee, Hydrothermal Synthesis of K2CO3Promoted Hydrotalcite from Hydroxide-Form Precursors for Novel High-Temperature CO2 Sorbent, ACS Applied Materials \& Interfaces, 6 (2014) 6914-6919.

[15] I. Khan, A.A.M. Ibrahim, M. Sohail, A. Qurashi, Sonochemical assisted synthesis of $\mathrm{RGO} / \mathrm{ZnO}$ nanowire arrays for photoelectrochemical water splitting, Ultrasonics Sonochemistry, 37 (2017) 669-675.

[16] S. Loganathan, M. Tikmani, A. Mishra, A.K. Ghoshal, Amine tethered pore-expanded MCM41 for CO2 capture: Experimental, isotherm and kinetic modeling studies, Chemical Engineering Journal, 303 (2016) 89-99.

[17] K.L. Tan, B.H. Hameed, Insight into the adsorption kinetics models for the removal of contaminants from aqueous solutions, Journal of the Taiwan Institute of Chemical Engineers, 74 (2017) 25-48.

[18] M.P. Bernardo, F.K.V. Moreira, L.A. Colnago, C. Ribeiro, Physico-chemical assessment of [Mg-Al-PO4]-LDHs obtained by structural reconstruction in high concentration of phosphate, Colloids and Surfaces A: Physicochemical and Engineering Aspects, 497 (2016) 53-62.

[19] A. Laskowska, M. Zaborski, G. Boiteux, O. Gain, A. Marzec, W. Maniukiewicz, Effects of unmodified layered double hydroxides MgAl-LDHs with various structures on the properties of filled carboxylated acrylonitrile-butadiene rubber XNBR, European Polymer Journal, 60 (2014) 172-185.

[20] H.W. Olfs, L.O. Torres-Dorante, R. Eckelt, H. Kosslick, Comparison of different synthesis routes for $\mathrm{Mg}-\mathrm{Al}$ layered double hydroxides $(\mathrm{LDH})$ : Characterization of the structural phases and anion exchange properties, Applied Clay Science, 43 (2009) 459-464.

[21] L. Dong, C. Ge, P. Qin, Y. Chen, Q. Xu, Immobilization and catalytic properties of candida lipolytic lipase on surface of organic intercalated and modified MgAl-LDHs, Solid State Sciences, 31 (2014) 8-15. 
[22] B. Li, Y. Zhang, X. Zhou, Z. Liu, Q. Liu, X. Li, Different dye removal mechanisms between monodispersed and uniform hexagonal thin plate-like MgAl-CO32--LDH and its calcined product in efficient removal of Congo red from water, Journal of Alloys and Compounds, 673 (2016) 265271.

[23] W. Lv, L. Yang, B. Fan, Y. Zhao, Y. Chen, N. Lu, R. Li, Silylated MgAl LDHs intercalated with $\mathrm{MnO} 2$ nanowires: Highly efficient catalysts for the solvent-free aerobic oxidation of ethylbenzene, Chemical Engineering Journal, 263 (2015) 309-316.

[24] H. Nina, A.R. Barron, BET Surface Area Analysis of Nanoparticles, Rice University, Houston TX, 2011.

[25] S. Brunauer, L.S. Deming, W.E. Deming, E. Teller, On a Theory of the van der Waals Adsorption of Gases, J. Am. Chem. Soc., 62 (1940) 1723-1732.

[26] K.S.W. Sing, D.H. Everett, R.A.W. Haul, L. Moscou, R.A. Pierotti, J. Rouquerol, S. T., Reporting Physisorption Data for Gas/Solid Systems with Special Reference to the Determination of Surface Area and Porosity, Pure \& Appl. Chem., 57 (1985) 603-619.

[27] T. Adschiri, Y. Hakuta, K. Arai, Hydrothermal Synthesis of Metal Oxide Fine Particles at Supercritical Conditions, Industrial and Engineering Chemistry Research, 39 (2000) 4901-4907.

[28] H. Xu, B.W. Zeiger, K.S. Suslick, Sonochemical synthesis of nanomaterials, Chemical Society reviews, 42 (2013) 2555-2567.

[29] Y. Kong, G. Jiang, Y. Wu, S. Cui, X. Shen, Amine hybrid aerogel for high-efficiency CO2 capture: Effect of amine loading and CO2 concentration, Chemical Engineering Journal, 306 (2016) $362-368$.

[30] R. Dolores, S. Raquel, G.-L. Adianez, Sonochemical synthesis of iron oxide nanoparticles loaded with folate and cisplatin: Effect of ultrasonic frequency, Ultrasonics Sonochemistry, 23 (2015) 391-398.

[31] E. Genty, J. Brunet, C. Poupin, S. Casale, S. Capelle, P. Massiani, S. Siffert, R. Cousin, Co-A1 mixed oxides prepared via LDH route using microwaves or ultrasound: Application for catalytic toluene total oxidation, Catalysts, 5 (2015) 851-867.

[32] J. Otsu, Y. Oshima, New approaches to the preparation of metal or metal oxide particles on the surface of porous materials using supercritical water: Development of supercritical water impregnation method, J. of Supercritical Fluids, 33 (2005) 61-67.

[33] M. Manikandan, A.K. Venugopal, K. Prabu, R.K. Jha, R. Thirumalaiswamy, Role of surface synergistic effect on the performance of Ni-based hydrotalcite catalyst for highly efficient hydrogenation of furfural, Journal of Molecular Catalysis A: Chemical, 417 (2016) 153-162. 
[34] N. Touisni, F. Charmantray, V. Helaine, C. Forano, L. Hecquet, C. Mousty, Optimized immobilization of transketolase from $\mathrm{E}$. coli in $\mathrm{MgAl}$-layered double hydroxides, Colloids and Surfaces B: Biointerfaces, 112 (2013) 452-459.

[35] S.-L. Wang, C.H. Liu, M.K. Wang, Y.H. Chuang, P.N. Chiang, Arsenate adsorption by $\mathrm{Mg} / \mathrm{Al}-\mathrm{NO} 3$ layered double hydroxides with varying the Mg/Al ratio, Applied Clay Science, 43 (2009) 79-85.

[36] M. Bellotto, B. Rebours, O. Clause, J. Lynch, D. Bazin, E. Elkaïm, A Reexamination of Hydrotalcite Crystal Chemistry, The Journal of Physical Chemistry, 100 (1996) 8527-8534.

[37] H. Ji, W. Wu, F. Li, X. Yu, J. Fu, L. Jia, Enhanced adsorption of bromate from aqueous solutions on ordered mesoporous $\mathrm{Mg}$-Al layered double hydroxides (LDHs), Journal of Hazardous Materials, 334 (2017) 212-222.

[38] S. Zhao, X. Meng, K. Zhu, F. Du, G. Chen, Y. Wei, Y. Gogotsi, Y. Gao, Li-ion uptake and increase in interlayer spacing of Nb4C3 MXene, Energy Storage Materials, 8 (2017) 42-48.

[39] H. Emadi, M. Salavati-Niasari, A. Sobhani, Synthesis of some transition metal (M: 25Mn, $27 \mathrm{Co}, 28 \mathrm{Ni}, 29 \mathrm{Cu}, 30 \mathrm{Zn}, 47 \mathrm{Ag}, 48 \mathrm{Cd}$ ) sulfide nanostructures by hydrothermal method, Advances in Colloid and Interface Science, DOI https://doi.org/10.1016/j.cis.2017.06.007.

[40] J.S. Valente, M. Sánchez-Cantú, E. Lima, F. Figueras, Method for Large-Scale Production of Multimetallic Layered Double Hydroxides: Formation Mechanism Discernment, Chemistry of Materials, 21 (2009) 5809-5818.

[41] S.-L. Wang, P.-C. Wang, In situ XRD and ATR-FTIR study on the molecular orientation of interlayer nitrate in $\mathrm{Mg} / \mathrm{Al}$-layered double hydroxides in water, Colloids and Surfaces A: Physicochemical and Engineering Aspects, 292 (2007) 131-138.

[42] L. Sciascia, M.L. Turco Liveri, M. Merli, Kinetic and equilibrium studies for the adsorption of acid nucleic bases onto K10 montmorillonite, Applied Clay Science, 53 (2011) 657-668.

[43] R. Serna-Guerrero, A. Sayari, Modeling adsorption of CO2 on amine-functionalized mesoporous silica. 2: Kinetics and breakthrough curves, Chemical Engineering Journal, 161 (2010) 182-190.

[44] J.M. Borah, J. Sarma, S. Mahiuddin, Adsorption comparison at the $\alpha$-alumina/water interface: 3,4-Dihydroxybenzoic acid vs. catechol, Colloids and Surfaces A: Physicochemical and Engineering Aspects, 387 (2011) 50-56.

[45] W. Plazinski, Applicability of the film-diffusion model for description of the adsorption kinetics at the solid/solution interfaces, Applied Surface Science, 256 (2010) 5157-5163.

[46] W. Plazinski, J. Dziuba, W. Rudzinski, Modeling of sorption kinetics: the pseudo-second order equation and the sorbate intraparticle diffusivity, Adsorption, 19 (2013) 1055-1064. 
Ultrasonic and Hydrothermal Mediated Synthesis Routes for Functionalized Mg-Al LDH: Comparison Study on Surface Morphology, Basic Site Strength, Cyclic Sorption Efficiency and Effectiveness

\author{
Collins I. Ezeh ${ }^{\mathrm{a}}$, Marco Tomatis ${ }^{\mathrm{a}}$, Xiaogang Yang ${ }^{\mathrm{a}, *}$, Jun $\mathrm{He}^{\mathrm{a}}$, Cheng-gong Sun ${ }^{\mathrm{b}, *}$ \\ ${ }^{a}$ Department of Mechanical, Materials and Manufacturing Engineering \\ University of Nottingham Ningbo \\ University Park, Ningbo 315100. P.R. China \\ ${ }^{\mathrm{b}}$ Department of Chemical and Environmental Engineering \\ University of Nottingham \\ University Park, Nottingham NG7 2RD, UK
}

\begin{abstract}
Amine functionalized layered double hydroxide (LDHs) adsorbents prepared using three different routes: co-precipitation, sono-chemical and ultrasonic-assisted high pressure hydrothermal. The prepared adsorbent samples were characterised using $\underline{X}$-ray diffraction (XRD), X-ray Photoelectron Spectroscopy (XPS), Scanning electron microscope-Energy dispersive X-ray spectroscopy (SEM-EDX), _Temperature Programmed Desorption (TPD), Brunauer-Emmett-Teller (BET), and Thermogravimetric analysis (TGA), respectively. The performance of the prepared adsorbents was tested under a controlled thermal-swing adsorption process to measure its adsorption capacity, regeneration and cyclic efficiencies subsequently. The characterisation results were compared with those obtained using the conventional preparation routes but taking the impact of sonochemical and hydrothermal pre-treatment on textural properties, adsorption capacity, regeneration and cyclic efficiencies into account. Textural results depicts a surge in surface area of the adsorbent synthesised by hydrothermal route $\left(311 \mathrm{~m}^{2} / \mathrm{g}\right)$ from 25 and $171 \mathrm{~m}^{2} / \mathrm{g}$ for conventional and ultrasonic routes respectively. Additionally, it has been revealed from the present study that adsorbents prepared using ultrasonic-assisted hydrothermal route exhibit a better $\mathrm{CO}_{2}$ uptake capacity than that prepared using sonochemical and conventional routes. Thus, the ultrasonic-assisted hydrothermal treatment can effectively promote the adsorption capacity of the adsorbent. This is probably due to the decrease of moderate (M-O) and weak ( $\mathrm{OH}^{-}$groups) basic sites with subsequent surge in the number of strong basic sites $\left(\mathrm{O}^{2-}\right)$ resulting from the hydrothermal process. Moreover, the cyclic adsorption efficiency of the ultrasonic mediated process was found to be $76 \%$ compared with $60 \%$ for conventional and 53\% for hydrothermal routes, respectively. According to the kinetic model analysis, adsorption mechanism is mostly dominated by physisorption before amine modification and chemisorption after the modification process.
\end{abstract}

Keywords: $\mathrm{CO}_{2}$ Adsorption, Layered Double Hydroxide, Ultrasound, Hydrothermal, Regeneration.

*Corresponding authors:

Tel: +86-574-88182419 E-mail: Xiaogang.Yang@nottingham.edu.cn (X. Yang)

Tel: +44-115-7484577 E-mail: cheng-gong.sun@ nottingham.ac.uk (C. Sun) 


\section{INTRODUCTION}

Adsorption of carbon dioxide is perceived as the most suitable method for Carbon Capture and Storage (CCS) technology $[1,2]$. Extensive studies on various adsorbents used for $\mathrm{CO}_{2}$ uptake has been carried out over the years. Layered double Hydroxides (LDHs) provided comparatively high uptake capacity with numerous catalytic applications [3, 4]. Additionally, its ionic interlayered configuration enhances the number of basic sites and provides high contact surface area [4]. LDHs are anionic clay minerals also known as mixed-metal layered hydroxides or hydrotalcites-like compounds. They possess two dimensional structure of layers shaped by

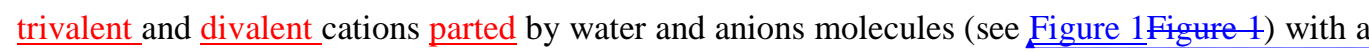
Formatted: Font: Not Bold general formula:

$$
\left(M_{1-x}^{2+} M_{x}^{3+}(\mathrm{OH})_{2}\right)^{x+}\left(A_{x / m}^{m-} \cdot n H_{2} O\right)^{x-}
$$

where $\mathrm{M}^{2+}$ and $\mathrm{M}^{3+}$ are divalent and trivalent cations respectively, $\mathrm{A}^{\mathrm{m}-}$ is a compensating anionic charge, $\mathrm{x}$ is the partial substitution of $\mathrm{M}^{2+}$ and $\mathrm{M}^{3+}$, usually within a $\mathrm{M}^{2+} / \mathrm{M}^{3+}$ ratio of 2 and 5 [46].

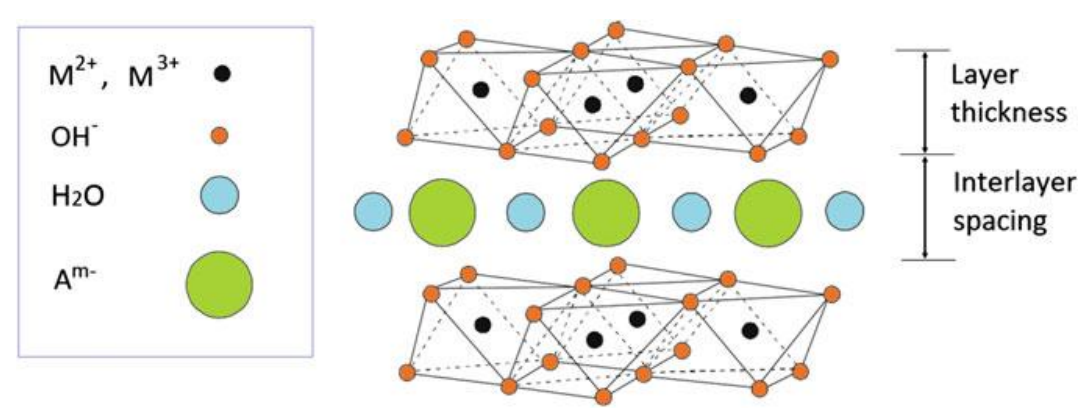

Figure 1: Structural configuration of layered double hydroxides ([5]-permission obtained)

LDHs are basically synthesized via co-precipitation of solutions containing $\mathrm{M}^{2+}$ and $\mathrm{M}^{3+}$ metal salts in a preferred interlayer anion [6,7]. This synthesis method can be conducted at high or low supersaturation environments. In low supersaturation condition, the metal solutions are added concurrently to the solution containing the alkali; whereas the metal (at a given $\mathrm{M}^{2+} / \mathrm{M}^{3+}$ ratio)

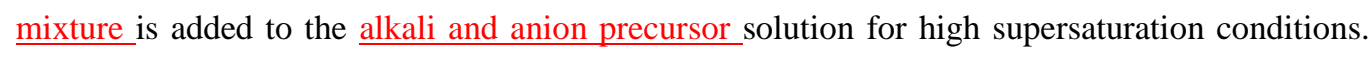
The former tends to produce more crystalline structures because of the increased rate of 
nucleation over crystallization [6]. Besides this method, other means of synthesizing LDH are sol-gel and urea techniques [7,8].

A key property of this material for the purpose of $\mathrm{CO}_{2}$ adsorption and other catalytic reactions is its basic property. Owing to the occurrence of interlayered anions and water molecules in the structure, LDH behave as solid bases. Wide ranges of basic strength can be obtained depending on the treatment route and metallic composition. This is primarily attributed to the nature and deposition of the divalent and trivalent cations on the material. With this property, $\mathrm{CO}_{2}$ has enough acidic strength to bond with the weak basic sites of LDH. However, with an improved basic strength, $\mathrm{CO}_{2}$ is chemisorbed weakly on low basic sites, surface hydroxyl groups forming bicarbonates and strongest on strong basic sites, free lattice oxygen $\left(\mathrm{O}^{2-}\right)$ forming mono-dentate carbonates. Adsorption on medium basic sites are on acid-base (metal-oxygen) pairs like $\mathrm{Mg}^{2+}$ $\mathrm{O}^{2-}$ or $\mathrm{Al}^{3+}-\mathrm{O}^{2-}$ forming tri-dentates or bi-dentates. The use of temperature-programmed desorption monitored by infrared spectroscopy are shown in Figure 2Figure 2 according to the Formatted: Font: Not Bold work [9].

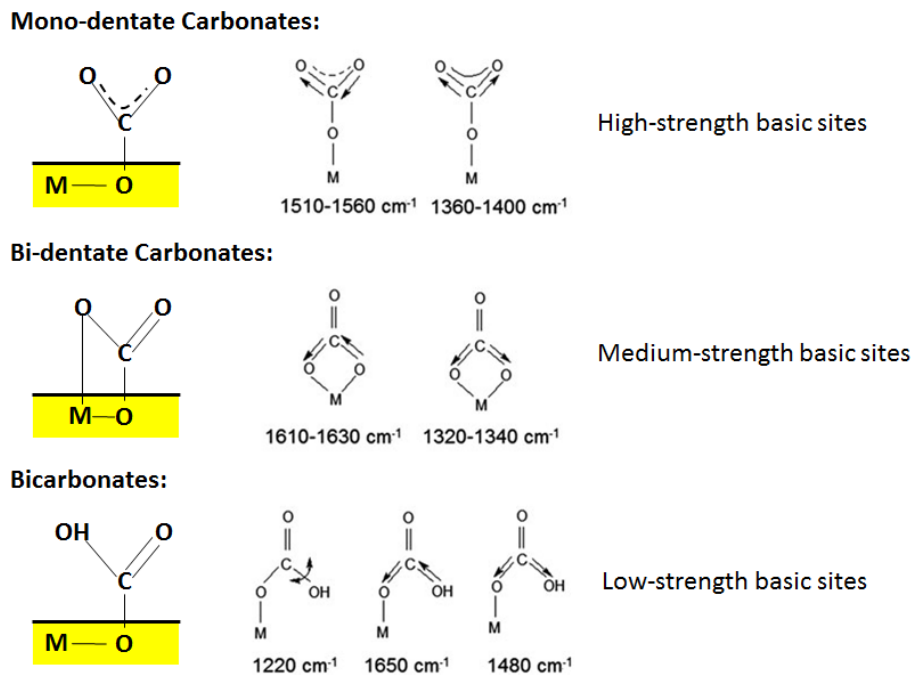

Figure 2: Infrared bands of adsorbed $\mathrm{CO}_{2}$ on LDH surface ([9]_Permission obtained) 
The strength of these basic sites determine the adsorption and regeneration capacity of the adsorbent. Most research studies, to the best our knowledge, have emphasised the effect of metallic composition on this property. However, it is observed from this study that the preparation route plays a key role in the basic strength of this material. Subsequently, this affects the adsorption and regeneration capacities, as well as the life span of the material [10]. In addition, the intimate contact between the metallic contents of the LDHs enhances the physicochemical properties of these materials making it suitable precursors for the synthesis of mixed oxides for hydrogenation and dehydrogenation reactions $[6,11]$. Metallic contact can be improved upon by boosting the deposition and dispersion of the cations on the adsorbent. This can be achieved by adopting several preparation routes [3, 12-14].

In this work, the impact of ultrasonic irradiation and ultrasonic-assisted hydrothermal synthesis route on the basicity, adsorption, regeneration and cyclic efficiencies were investigated. This is a follow-up study to recent work [10]. This method has shown to produce materials with wellordered formation [15]. The prepared samples were characterized using Scanning Electron Microscope (SEM), Energy Dispersion X-ray Spectroscopy (EDX), Brunauer-Emmett-Teller (BET), Thermal Gravimetric Analyzer (TGA), Temperature-Programmed Desorption (TPD), Xray Photoelectron Spectroscopy (XPS) and X-ray Diffraction (XRD).

\section{EXPERIMENTAL}

\subsection{Material preparation}

Different preparation methods were used in preparing the layered double hydroxides (LDHs) using the standard method from previous study [10]: conventional co-precipitation, ultrasonic mediated and ultrasonic-assisted hydrothermal. Subsequently, MEA extractions of these LDHs were carried out to produce the amine modified LDHs. All chemicals used for experimentation were acquired from SinoPharm Chemical Reagents Co. Ltd.

\subsection{Sample Synthesis}

For $\mathrm{MgAl} \mathrm{LDH}, 200 \mathrm{ml}$ solution containing APTS ( $\geq 98 \%$ ) and SDS ( $\geq 86 \%$ ) (molar ratio: 5:1) respectively dissolved in a mixture of $50 \mathrm{ml} \mathrm{C}_{2} \mathrm{H}_{5} \mathrm{OH}(\geq 99.7 \%)$ and $150 \mathrm{ml}$ distilled water was stirred for about $30 \mathrm{~min}$ at a temperature of $60{ }^{\circ} \mathrm{C}$ until the $\mathrm{pH}$ stabilized at about 10.3. This 
solution was then reacted with $\mathrm{Mg}\left(\mathrm{NO}_{3}\right)_{2} \cdot 6 \mathrm{H}_{2} \mathrm{O}(\geq 98 \%)$ and $\mathrm{Al}\left(\mathrm{NO}_{3}\right)_{3} \cdot 9 \mathrm{H}_{2} \mathrm{O}(\geq 99 \%)$ (molar ratio: 3:1, dissolved in $100 \mathrm{ml}$ of distilled water) solution by adding the latter dropwise while maintaining the temperature of the former at $60{ }^{\circ} \mathrm{C}$. The mixture $\mathrm{pH}$ was regulated towards 10 using $4 \mathrm{M} \mathrm{NaOH}(\geq 96 \%)$ solution. The substrate (molar ratio of Mg:Al:APTS:SDS = 3:1:5:1) was then aged for $20 \mathrm{hr}$ with the constant stirring at the maintained temperature. The filtered precipitates were then washed with ionised water before being vacuum dried $\left(500 \mathrm{mbar}\right.$ at $\left.70{ }^{\circ} \mathrm{C}\right)$ overnight. This sample is labelled as Conv-LDH. Using the same chemical composition and process, a set of new samples were prepared using sonicated mixing by ultrasonic horns (high intensity sonication, 600W)_[10]. This sample is labelled as US-LDH.

For ultrasonic-assisted hydrothermal synthesized samples, HPH-LDH, $32 \mathrm{ml}$ solution containing APTS ( $\geq 98 \%$ ) and SDS ( $\geq 86 \%$ ) (APTS:SDS molar ratio: 5) was reacted with $\mathrm{Mg}\left(\mathrm{NO}_{3}\right)_{2} \cdot 6 \mathrm{H}_{2} \mathrm{O}$ $(\geq 98 \%)$ and $\mathrm{Al}\left(\mathrm{NO}_{3}\right)_{3} \cdot 9 \mathrm{H}_{2} \mathrm{O}(\geq 99 \%)$ (Mg:Al molar ratio: 3, dissolved in $16 \mathrm{ml}$ of distilled water) solution. The latter was added dropwise while maintaining the temperature of the former at $60{ }^{\circ} \mathrm{C}$ and sonicating with ultrasonic horn. The substrate (molar ratio of Mg:Al:APTS:SDS = 3:1:5:12 was moved to the hydrothermal reactor. After being vented with pure $\mathrm{N}_{2}$ gas (99.99\%) for $15 \mathrm{mins}$ in order to remove all of the oxygen before the reaction, the reactor was sealed and heated to a temperature of $450{ }^{\circ} \mathrm{C}$ with a ramp of $2{ }^{\circ} \mathrm{C} / \mathrm{min}$ and then held for $15 \mathrm{mins}$. After reaction, the solution was aged for $12 \mathrm{hr}$. The precipitates were filtered, washed with distilled water and then dried in a vacuum oven $\left(500 \mathrm{mbar}\right.$ at $\left.70{ }^{\circ} \mathrm{C}\right)$ for $7 \mathrm{~h}$.

For $M g A l L D H-M E A$, the prepared LDHs were functionalised via MEA extraction. $0.5 \mathrm{~g}$ of LDH sample was dispersed in a solution of $100 \mathrm{ml} \mathrm{C}_{2} \mathrm{H}_{5} \mathrm{OH}(\geq 99.7 \%)$ containing $20 \mathrm{~g}$ MEA ( $\left.\geq 99 \%\right)$. The mixture was then refluxed for $20 \mathrm{hr}$ at a temperature of $90{ }^{\circ} \mathrm{C}$. After which the samples were filtered, washed with ethanol and dried in a vacuum oven overnight. These samples are labelled Conv-MEA. Using an ultrasonic horn, the procedure was repeated for the synthesised US-LDHn samples. The obtained samples are labelled US-MEA. Similarly, this was repeated for the HPHLDH samples and labelled as HPH-MEA. 


\subsection{Characterisation}

2.3.1. Nitrogen adsorption-desorption analysis:

The textural characteristics of the prepared catalysts were studied by Nitrogen physisorption analysis at $-196{ }^{\circ} \mathrm{C}$ using the Micrometrics ASAP 2020 Surface Area and Porosity Analyser. Prior to this analysis, samples were degassed at a temperature of $105_{-}^{\circ} \mathrm{C}$ for $4 \_$hr. The surface area ( $\mathrm{S}_{\mathrm{BET}}$ ) of the samples was determined using the BET (Brunauer, Emmett and Teller) model. The total pore volumes $\left(\mathrm{V}_{\text {Total }}\right)$ were computed from the amount of nitrogen adsorbed at relative pressure $\left(\mathrm{P} / \mathrm{P}_{\mathrm{o}}\right)$ of 0.99 and the average pore volumes from $4 \mathrm{~V}_{\text {Total }} / \mathrm{S}_{\mathrm{BET}}$. The pore size distribution was calculated using the BJH (Barrett, Joyner and Halenda) model. The t-plot method was used to calculate the micopore volume $\left(\mathrm{V}_{\text {micro }}\right)$.

\subsubsection{Scanning electron microscope-Energy dispersive X-ray spectroscopy (SEM-EDX):}

The surface composition and structure of the prepared materials were studied with a Zeiss IIGMA $^{\text {тм }}$ Field Emission SEM and an Oxford Instrument INCAx-act PentaFET® Precision EDX. The EDX spectra for the LDHs were obtained. This was also used to compute the amine content present in the adsorbents

\subsubsection{X-ray Diffraction (XRD) and X-ray Photoelectron Spectroscopy (XPS) Analysis:}

XRD patterns were studied using a Bruker-AXS D8 advance powder diffractometer with a scanning range of $10^{\circ} \leq 2 \theta \leq 90^{\circ}$. X-ray Photoelectron Spectroscopy (XPS) data of the adsorbent was obtained using Kratos X-ray Photoelectron Spectrometer - Axis Ultra DLD with a $96 \mathrm{~W}$ monochromatic Al Ka X-ray source $(1486.69 \mathrm{eV})$ at a photoelectron take-off angle of $45^{\circ}$. Narrow scans were conducted at steps of $0.05 \mathrm{eV}$ with dwell time of $600 \mathrm{~ms}$ whereas wide scans were conducted from $1100 \mathrm{eV}$ to $0 \mathrm{eV}$ with $\underline{150 \mathrm{~ms}}$ dwell time and at $1 \mathrm{eV}$ steps. The $\underline{\mathrm{C}} 1 \mathrm{~s}$ peak at $284.6 \mathrm{eV}$ was used as the reference for standardizing the binding energy.

\subsubsection{Temperature-Programmed Desorption (TPD):}

$\mathrm{CO}_{2}$-TPD analysis was conducted using AutoChem II 2920. The TPD of $\mathrm{CO}_{2}$ measurements were conducted to evaluate the basicity of the catalysts. $0.1 \mathrm{~g}$ of the adsorbent was treated in the $\underline{\text { reactor in } \mathrm{N}_{2} \text { atmosphere at a temperature of } 350{ }^{\circ} \mathrm{C} \text { for } 2 \mathrm{hr} \text {. A thermal conductivity detector }}$ 
(TCD) was adopted to analyze desorption trends from 100 to $800{ }^{\circ} \mathrm{C}$ at a heating rate of $10^{\circ} \mathrm{C} / \mathrm{min}$.

\subsubsection{Thermo-gravimetric analysis:}

Thermal stability and $\mathrm{CO}_{2}$ uptake measurements were conducted using the Netzsch STA 449 F3 Jupiter thermogravimetric analyser (TGA).

Thermal Stability Measurement. The stability of the as-synthesised LDH samples in air was

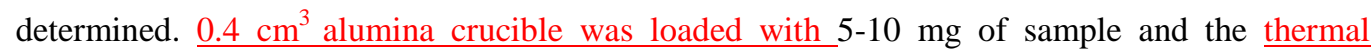
decomposition examined from 25 to $1000{ }^{\circ} \mathrm{C}$, ramped $10^{\circ} \mathrm{C} / \mathrm{min}$ and under air flow $(50 \mathrm{ml} / \mathrm{min})$.

$\mathrm{CO}_{2}$ Uptake Measurement. Approximately 5-10 mg of the synthesized sample was heated from 25 to $105^{\circ} \mathrm{C}$ at $20{ }^{\circ} \mathrm{C} / \mathrm{min}$ under $\mathrm{N}_{2}$ at a flow rate of $50 \mathrm{ml} / \mathrm{min}$; and then held isothermally for 30 min before being cooled to the required adsorption temperature at a rate of $10{ }^{\circ} \mathrm{C} / \mathrm{min}$. The gas was switched from $\mathrm{N}_{2}$ to $\mathrm{CO}_{2}$ and held isothermally for $90 \mathrm{~min}$ at a flow rate of $20 \mathrm{ml} / \mathrm{min}$. The experimental adsorption temperature is $55^{\circ} \mathrm{C}$. The $\mathrm{CO}_{2}$ adsorption capacity was calculated from the weight difference of the samples in $\mathrm{CO}_{2}$ atmosphere. The uptake profile was measured against an empty alumina crucible using the same method so as to correct the impact of change in gas density and viscosity.

Thermally Assisted Regeneration of Adsorbent. Regeneration efficiency of the functionalized samples were analysed by thermal-assisted regeneration using nitrogen as a sweep gas. Two cycles of the $\mathrm{CO}_{2}$ uptake measurement was repeated in this case. In between the cycles, the adsorbents were thermally desorbed at $105{ }^{\circ} \mathrm{C}$ in a stream of $\mathrm{N}_{2}$ at a flow rate of $50 \mathrm{ml} / \mathrm{min}$ for 10 mins. The amount adsorbed $\left(\mathrm{Q}_{\mathrm{a}}\right)$ and desorbed $\left(\mathrm{Q}_{\mathrm{d}}\right)$ were deduced by the change in weight of the samples. The regeneration efficiency (RE) was thus estimated based on equation (1):

$$
R E=\frac{Q_{d}}{Q_{a}} \times 100 \% \quad \text { (Equation-1) }
$$




\section{THEORETICAL}

To predict kinetic behaviour, the experimental results were simulated assuming pseudo-first, pseudo-second order and double-exponential kinetic models_[16]. The double exponential model was considered because of its feasibility to explain the surface heterogeneity of the adsorbent accounting for two different adsorption sites. Additionally, it can partially elaborate the kinetic mechanisms associated with both pseudo-first and second order reactions. This mechanism can be categorised into a controlled slow phase intra-particle diffusion and a controlled fast phase surface reaction [17].

\section{Pseudo-first order model}

$$
x=x_{e}\left(1-e^{-k_{f} t}\right)
$$

\section{$\underline{\text { Pseudo-second order model }}$}

$$
x=\frac{x_{e}^{2} k_{s} t}{1+x_{e} k_{s} t}
$$

\section{Double exponential model}

$$
x=x_{e}-\left(A_{1} e^{-k_{1} t}+A_{2} e^{-k_{2} t}\right)
$$

where $x$ and $x_{e}$ represents the $\mathrm{CO}_{2}$ uptake at a given time and equilibrium respectively, $k_{f}$ and $k_{s}$ are the first and second order rate constants respectively, $A_{i}$ and $k_{i}, i=1,2$ are pre-exponential factors and rate constants for the two adsorption sites respectively, and $t$ is the time of adsorption. The obtained experimental data are fitted to the models and selecting the one with the best fit.

To determine the suitability of each model, an error function (Err) defined by equation (5) was applied:

$$
\operatorname{Err}(\%)=\sqrt{\frac{\sum_{j=1}^{N}\left[\frac{x_{j, \mathrm{exp}}-x_{j, \mathrm{mmd}}}{x_{j, \mathrm{exp}}}\right]^{2}}{N-1}} \times 100
$$

(Equation-5)

where $\mathrm{x}_{\text {exp }}$ and $\mathrm{x}_{\mathrm{mod}}$ are $\mathrm{CO}_{2}$ uptake determined experimentally and computed using the model respectively and $\mathrm{N}$ is the total number of experimental points. It is reasonable to assume that the adsorption rate constant, $\mathrm{k}$ for both pseudo-first and -second order model is the same for each 
group of functionalized and non-functionalized adsorbents since they are both grafted with the same amino silane. However, this was not assumed in this study. The effect of preparation route on the rate constant was rather studied

\section{DISCUSSION OF RESULTS}

\subsection{Impact of preparation route on textural properties and surface morphology}

Table 1: Texture properties of the samples

\begin{tabular}{lcccccc}
\hline \multirow{2}{*}{ Sample } & $\mathbf{S}_{\text {BET }}$ & $\begin{array}{c}\text { Particle } \\
\text { Size }\end{array}$ & $\mathbf{V}_{\text {Total }}$ & $\mathbf{V}_{\text {micro }}$ & $\begin{array}{c}\text { Average Pore } \\
\text { Width }\end{array}$ & \% Micro* \\
\cline { 2 - 7 } & $\mathbf{( \mathbf { m } ^ { 2 } / \mathbf { g } )}$ & $\mathbf{( n m )}$ & $\mathbf{( \mathbf { c m } ^ { 3 } / \mathbf { g } )}$ & $\mathbf{( \mathbf { c m } ^ { 3 } / \mathbf { g } )}$ & $\mathbf{( n m )}$ & $\mathbf{( \% )}$ \\
\hline Conv-LDH & 25.03 & 239.67 & 0.0161 & 0.0008 & 2.57 & 4.97 \\
US-LDH & 171.20 & 35.05 & 0.5528 & 0.0229 & 12.92 & 4.14 \\
HPH-LDH & 311.18 & 19.28 & 1.4005 & 0.0023 & 18.00 & 0.16 \\
\hline
\end{tabular}

$* \%$ Micro $=V_{\text {micro }} V_{\text {Total }}$

From Table 1Table 1, $\mathrm{S}_{\mathrm{BET}}$ of Conv-LDH is $c a .25 \mathrm{~m}^{2} / \mathrm{g}$ which showed an increase to 171.20 and $311.18 \mathrm{~m}^{2} / \mathrm{g}$ for US-LDH and HPH-LDH respectively. In the same trend, the total pore volume showed a tremendous increase indicating that the sonication and hydrothermal preparation methods enhances the porosity of the sample as well as the surface area when compared to other studies (see Table 2Table 2). However, these methods tend to reduce the micropores in the sample with the hydrothermal route exhibiting the least microporous percentile. This depicts that the sample is a mesoporous/macroporous material with minor microporous features. The features of these pores can be seen from Figure 3. All three samples contained approximately the same volume ratio of mesopores to macropores. However, the Conv-LDH is observed to have a higher percentile of low range mesopores $(2-10 \mathrm{~nm})$ contributing to $55 \%$ of the total pore volume as compared to $10 \%$ and $15 \%$ for US-LDH and HPH-LDH respectively. The high range mesopores $(10-50 \mathrm{~nm})$ accounted for about $50 \%$ of the total pore volume of the US-LDH and HPH-LDH samples compared to $c a$. $7 \%$ in Conv-LDH. This can be further illustrated by the inserted image of Figure 3Figure 3 displaying the pore size distribution in terms of total volume percentages.

Formatted: Font: Not Bold

Formatted: Font: Not Bold 


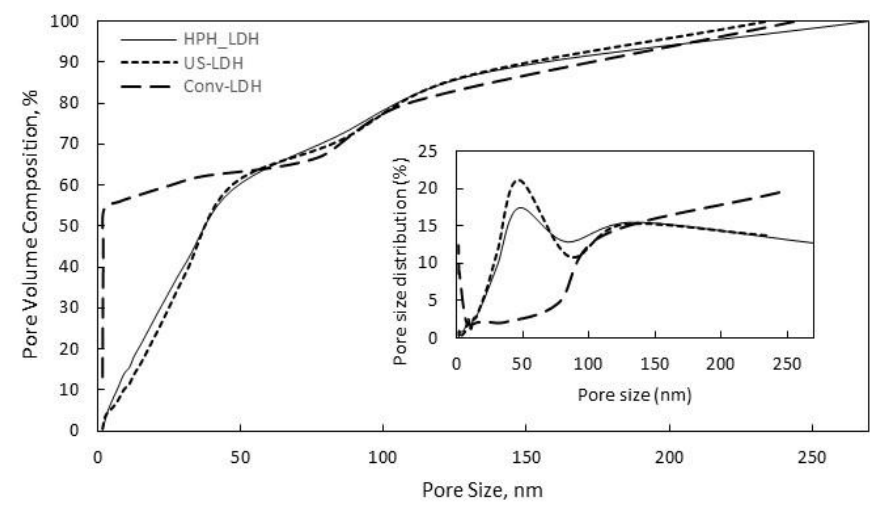

Figure 3: Pore size distribution of Conv-, US- and HPH-LDH samples

Figure 4Figure 4 shows the total absolute volume of the pores at various pore size. It depicts that Formatted: Font: Not Bold the high range mesopores mostly contribute to the total pore volume of the samples and comprises of a higher pore distribution.

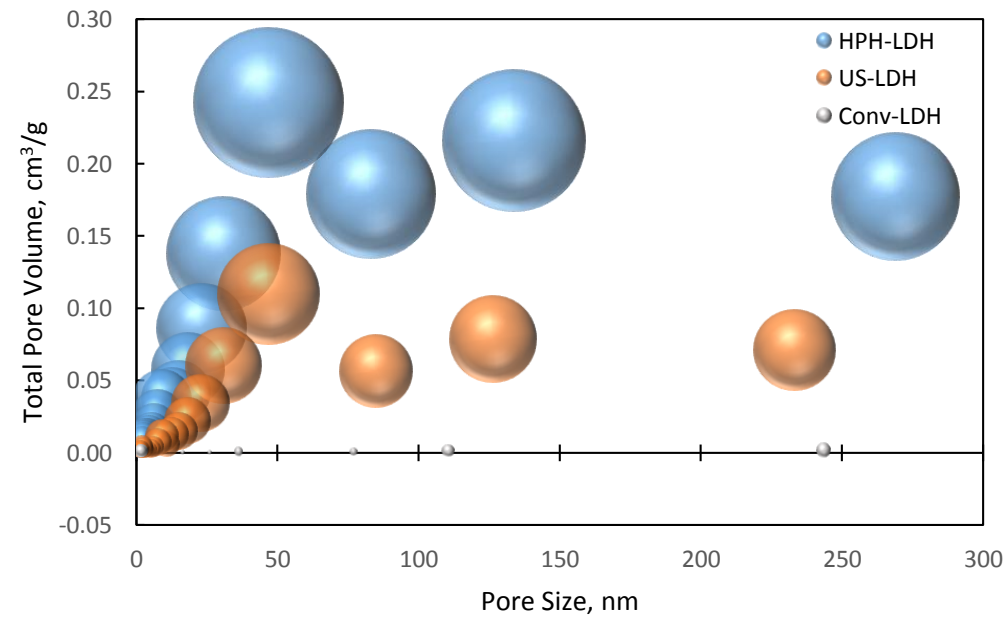

Figure 4: Total pore volume of various pore sizes of Conv-, US- and HPH-LDH samples (bubble size indicates relative amount of pore size) 
Table 2: Comparison of $S_{B E T}$ for different $L D H$ adsorbent via different preparation routes

\begin{tabular}{|c|c|c|c|}
\hline Sample* & Preparation Method & $\begin{array}{l}\text { SBET } \\
\left(\mathbf{m}^{2} / \mathbf{g}\right)\end{array}$ & Reference \\
\hline$L D H$ & Commercial & 2.90 & {$[18]$} \\
\hline $\mathrm{CO}_{3}-\mathrm{LDH}$ & Commercial & 18.90 & [19] \\
\hline Conv-LDH & Co-precipitation & 10.50 & [20] \\
\hline Conv-LDH & Co-precipitation & $44-70$ & [18] \\
\hline Conv-LDH & Co-precipitation & 69.78 & [21] \\
\hline Conv-LDH & Co-precipitation & 25.03 & Current work \\
\hline$c-L D H$ & Calcined Co-precipitation & $84-123$ & [18] \\
\hline$c-\mathrm{CO}_{3}-\mathrm{LDH}$ & Calcined Solvothermal & 165.07 & [22] \\
\hline$U S-L D H$ & Ultrasonic assisted & 84.44 & [21] \\
\hline$U S-L D H$ & Ultrasonic assisted & 171.20 & Current work \\
\hline$r-L D H$ & Reconstructed & $45-55$ & [18] \\
\hline $\mathrm{CO}_{3}-\mathrm{LDH}$ & Solvothermal & 24.74 & [22] \\
\hline$L D H$ & Silylated-Calcined & 19.20 & [23] \\
\hline$H P H-L D H$ & Hydrothermal & 311.18 & Current work \\
\hline
\end{tabular}

*LDH samples are $M g-A l L D H$ 


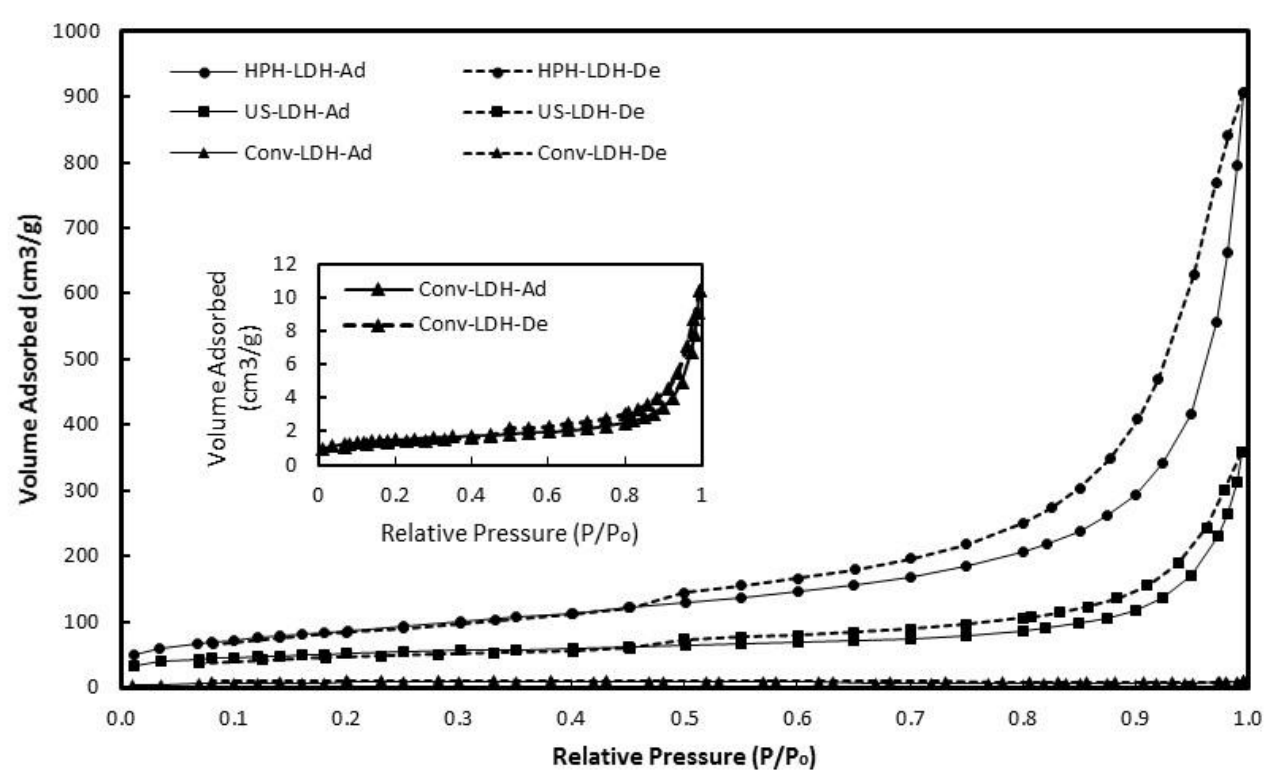

Formatted: Font: (Default) Times New Roman, 12 pt, Font color: Text 1

Figure 5: Nitrogen adsorption-desorption isotherms for Conv-LDH, US-LDH and HPH-LDH samples (inserted image shows the enlarged isotherm for Conv-LDH) 
Table 3: Surface content and binding energy for $N 1 s$ and $O$ 1s levels for $L D H, U S-L D H$ and HPH-LDH

\begin{tabular}{|c|c|c|c|c|c|c|c|c|c|c|c|}
\hline \multirow[b]{3}{*}{$\begin{array}{l}\text { Preparation } \\
\text { Route }\end{array}$} & \multirow[b]{3}{*}{ Sample } & \multicolumn{5}{|c|}{ N 1s Spectra } & \multicolumn{5}{|c|}{ O 1s Spectra } \\
\hline & & \multicolumn{2}{|c|}{$\begin{array}{c}\text { Binding Energy } \\
(\mathrm{eV})\end{array}$} & \multicolumn{2}{|c|}{$\begin{array}{c}\text { Area } \\
(\text { cps eV) }\end{array}$} & \multirow{2}{*}{$\begin{array}{c}\text { Content } \\
\left(\frac{\text { Free }}{\text { Protonated }}\right)\end{array}$} & \multicolumn{2}{|c|}{$\begin{array}{c}\text { Binding Energy } \\
(\mathrm{eV})\end{array}$} & \multicolumn{2}{|c|}{$\begin{array}{c}\text { Area } \\
(\operatorname{cps} \mathrm{eV})\end{array}$} & \multirow{2}{*}{$\begin{array}{l}\text { Content } \\
\left(\frac{O H^{-}}{O^{2-}}\right)\end{array}$} \\
\hline & & $\begin{array}{l}\text { Free } \\
\text { Amine }\end{array}$ & $\begin{array}{l}\text { Protonated } \\
\text { Amine }\end{array}$ & $\begin{array}{c}\text { Free } \\
\text { Amine }\end{array}$ & $\begin{array}{l}\text { Protonated } \\
\text { Amine }\end{array}$ & & $\begin{array}{l}\text { Lattice } \\
\text { Oxygen } \\
\left(\mathrm{O}^{2-}\right)\end{array}$ & $\begin{array}{c}\text { Hydroxyl } \\
\text { Group } \\
\left(\mathrm{OH}^{-}\right)\end{array}$ & $\begin{array}{l}\text { Lattice } \\
\text { Oxygen } \\
\left(\mathrm{O}^{2-}\right)\end{array}$ & $\begin{array}{l}\text { Hydroxyl } \\
\text { Group } \\
\left(\mathrm{OH}^{-}\right)\end{array}$ & \\
\hline Conventional & Conv-LDH & 396.7 & 400.8 & 640.0 & 626.4 & 1.02 & 529.9 & 531.4 & 7633.2 & 1011.8 & 0.13 \\
\hline Ultrasonic & US-LDH & 396.9 & 400.3 & 574.1 & 876.6 & 0.65 & 529.8 & 531.3 & 10109.2 & 2053.1 & 0.21 \\
\hline Hydrothermal & HPH-LDH & 395.4 & 399.1 & 349.7 & 791.2 & 0.44 & 530.4 & - & 60510.2 & - & - \\
\hline
\end{tabular}

Table 4: EDX elemental analysis and CO2 uptake of prepared LDH and calculation results for the molecular formulas, removed SDS and effective amine efficiency

\begin{tabular}{|c|c|c|c|c|c|c|c|c|c|c|}
\hline Sample & $\begin{array}{c}\mathrm{N} \\
(\mathrm{wt} \%)\end{array}$ & $\begin{array}{c}C \\
(w t \%)\end{array}$ & $\begin{array}{c}S \\
(w t \%)\end{array}$ & $\begin{array}{l}\text { Molecular formula } \\
\quad(\mathbf{m m o l} / \mathbf{g})^{\mathrm{a}}\end{array}$ & SDS/APTS & $\begin{array}{c}\text { Amine } \\
\text { Loading } \\
(\text { mmol/g) }\end{array}$ & $\begin{array}{c}\mathrm{CO}_{2} \\
\text { Adsorbed } \\
\text { (mmol/g) }\end{array}$ & $\begin{array}{c}\text { SDS } \\
\text { removed } \\
(\%)\end{array}$ & $\begin{array}{c}\text { Effective } \\
\text { amine } \\
\text { loading } \\
(\mathrm{mmol} / \mathrm{g})^{\mathbf{b}}\end{array}$ & $\begin{array}{l}\text { Effective } \\
\text { Amine } \\
\text { Efficiency }\end{array}$ \\
\hline Conv-LDH & 0.65 & 51.35 & 11.41 & 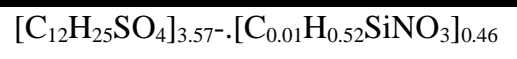 & 7.68 & 0.46 & 0.82 & & & \\
\hline Conv-MEA & 6.60 & 26.60 & 1.05 & 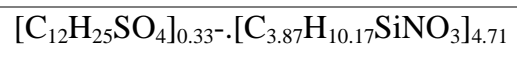 & 0.07 & 4.71 & 1.45 & 99.09 & 4.25 & 0.15 \\
\hline US-LDH & 3.11 & 51.16 & 11.08 & 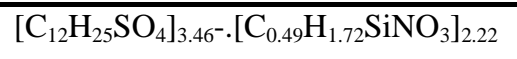 & 1.56 & 2.22 & 0.66 & & & \\
\hline US-MEA & 7.34 & 30.64 & 0.60 & {$\left[\mathrm{C}_{12} \mathrm{H}_{25} \mathrm{SO}_{4}\right]_{0.19^{-}-}\left[\mathrm{C}_{4.44} \mathrm{H}_{11.60} \mathrm{SiNO}_{3}\right]_{5.24}$} & 0.04 & 5.24 & 1.37 & 97.71 & 3.02 & 0.24 \\
\hline HPH-LDH & 2.55 & 13.68 & 1.50 & 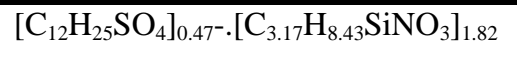 & 0.26 & 1.82 & 0.88 & & & \\
\hline HPH-MEA & 7.36 & 9.27 & 0.82 & 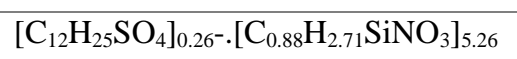 & 0.05 & 5.26 & 3.75 & 81.06 & 3.44 & 0.83 \\
\hline
\end{tabular}

a. All Nitrogen, Carbon and Sulphur elements in the grafted LDHs were attributed to come from organic compounds used for intercalation

b. Effective amine loading = Difference in amine loading before and after LDH modification

c. Effective Amine Efficiency = Difference in $\mathrm{CO}_{2}$ adsorbed before and after LDH modification/effective amine loading 
Table 5: $\mathrm{CO}_{2}$ kinetic model parameters, $R^{2}$ and standard errors (\%) for prepared $L D H s$ and amine functionalized $L D H s$ at $55^{\circ} \mathrm{C}$ and $1 \mathrm{~atm}$

\begin{tabular}{|c|c|c|c|c|c|c|c|c|c|c|c|c|c|c|c|}
\hline \multirow{2}{*}{ Samples } & \multicolumn{2}{|c|}{$\begin{array}{c}\text { Pseudo 1st } \\
\text { Order } \\
\end{array}$} & \multirow{2}{*}{$\begin{array}{l}\text { Err } \\
(\%)\end{array}$} & \multirow[t]{2}{*}{$\mathbf{R}^{2}$} & \multicolumn{2}{|c|}{$\begin{array}{c}\text { Pseudo 2nd } \\
\text { Order } \\
\end{array}$} & \multirow{2}{*}{$\begin{array}{l}\text { Err } \\
(\%)\end{array}$} & \multirow[t]{2}{*}{$\mathbf{R}^{2}$} & \multicolumn{5}{|c|}{ Double Exponential } & \multirow{2}{*}{$\begin{array}{l}\text { Err } \\
(\%)\end{array}$} & \multirow[t]{2}{*}{$\mathbf{R}^{2}$} \\
\hline & $\mathbf{x}_{\mathbf{e}}$ & $\mathbf{k}_{\mathbf{f}}$ & & & $\mathbf{x}_{\mathbf{e}}$ & $\mathbf{k}_{\mathrm{s}}$ & & & $\overline{\mathbf{x}_{\mathrm{e}}}$ & $\overline{A_{1}}$ & $\overline{A_{2}}$ & $\mathbf{k}_{1}$ & $\mathbf{k}_{2}$ & & \\
\hline Conv-LDH & 0.75 & 0.12 & 0.07 & 0.9766 & 0.83 & 0.23 & 0.34 & 0.9483 & 0.76 & 0.31 & 0.55 & 0.31 & 0.10 & 3.71 & 0.9815 \\
\hline US-LDH & 0.55 & 0.09 & 0.37 & 0.9062 & 0.63 & 0.20 & 0.81 & 0.9631 & 0.85 & 0.47 & 0.45 & 0.01 & 0.23 & 9.24 & 0.9897 \\
\hline HPH-LDH & 0.89 & 0.05 & 0.14 & 0.9356 & 1.14 & 0.04 & 0.38 & 0.9034 & 0.88 & 0.48 & 0.48 & 0.06 & 0.06 & 0.28 & 0.9421 \\
\hline Conv-MEA & 1.33 & 0.13 & 0.10 & 0.8786 & 1.46 & 0.14 & 0.08 & 0.9711 & 1.41 & 0.75 & 0.67 & 0.05 & 0.69 & 0.41 & 0.9968 \\
\hline US-MEA & 1.32 & 0.05 & 0.10 & 0.9826 & 1.67 & 0.03 & 0.28 & 0.9709 & 1.30 & 0.70 & 0.70 & 0.06 & 0.06 & 0.09 & 0.9861 \\
\hline НРН-MEA & 3.52 & 0.35 & 2.06 & 0.8733 & 3.72 & 0.17 & 2.34 & 0.9155 & 3.58 & 1.63 & 2.04 & 0.14 & 1.07 & 2.01 & 0.9327 \\
\hline
\end{tabular}


Figure 5Figure 5 shows the $\mathrm{N}_{2}$ adsorption-desorption isotherms for Conv-LDH, US-LDH and

HPH-LDH samples. The adsorbents show a type IV isotherm with H1 hysteresis loop characterized with the formation of a monolayer accompanied by multilayers occurring as a result of capillary condensation. This is a characteristic feature of mesoporous materials [24-26] validating the data represented in Table 1 Fable 1 . These H1-hysteresis type materials are attributed to the formation of aggregates or compact uniform spheres as shown in the SEM images [26]. The hysteresis loop for the HPH-LDH was observed to be broader than that of US- and Conv-LDHs indicating the extent of capillary condensation in the samples. It is observed that the volume adsorbed by HPH-LDH is 3 folds that of US-LDH, which in turn is $\sim 30$ folds of the conventional LDH. This depicts an enhancement in the pore structure of the samples and is attributed to the enhancement of nucleation and precipitate growth resulting from improved dissolution and reaction process via sonication [20] and hydrothermal crystallization [27].
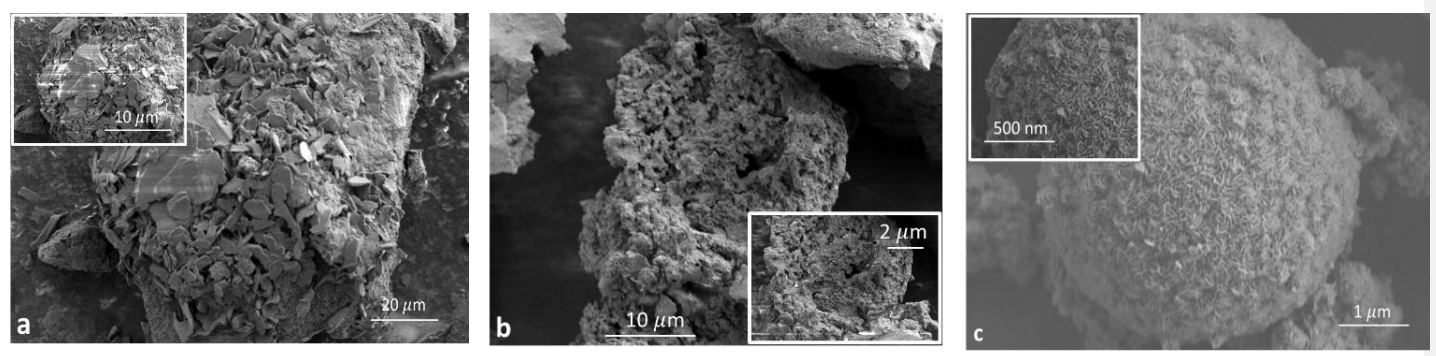

Figure 6: SEM images of prepared LDHs (a) Conv-LDH, (b) US-LDH, and (c) HPH-LDH

Considering the surface texture of the samples, Figure 6 shows images of surface morphology of the prepared LDH samples. It can be seen from the figure that Conv-LDH presents a bouldershaped material with aggregated flaky plate-like surface. This may be explained by the formation of shell-core structure caused by the sequential reduction of two different metallic ions $[10,28]$ resulted from the difference in the reduction potentials of $\mathrm{Al}^{3+}$ and $\mathrm{Mg}^{2+}$ ions. This is similar to the sonication route sample but without the plate-like surface. Surface of these samples displayed more pores attributable to high-speed jets or shock waves resulting from bubble collapse [28]. Unlike the conventionally and ultrasonically prepared samples, the hydrothermal LDHs were smaller and of spherical structure, hence increasing the effective surface area for adsorption. 


\subsection{Impact of preparation route on surface chemical content}

To investigate the impact of preparation route on the amine functional group and extent of oxidation on the surface of the adsorbent, XPS analysis was conducted. The N 1s and O 1s spectra are shown in Figure 7Figure 7 and the data are summarized in Table 3Fable 3. The deconvolution of $\mathrm{N} 1 \mathrm{~s}$ spectra has two peaks at $c a .396( \pm 0.9) \mathrm{eV}$ and $400( \pm 0.9) \mathrm{eV}$ respectively (Figure 7Figure 7a). These are assigned to free amine and protonated amines respectively [10, 29]. The concentration of free amino group decreased for species prepared via ultrasonic and hydrothermal mediation. This reveals that despite the enhanced physical properties of the adsorbent, ultrasonic and hydrothermal process has a reduced amount of free amino active sites on the adsorbent. This can be explained by the enhanced protonation of the free amine groups. However, the amine loading (deduced by the total concentration of $\mathrm{N}$ atom) is highest in species prepared via ultrasonic irradiation and least in the hydrothermal process. This can be partially attributed to the efficient ejection of hydroxyl radicals from bubbles during cavitation, which readily hydrates the anions; hence bonding the anions within the interlayers of the adsorbent [30]. This can be supported by the high content of hydroxyl group $\left(\mathrm{OH}^{-}\right)$in samples synthesised via ultrasonic irradiation. Similar to the $\mathrm{N} 1 \mathrm{~s}$ spectra, Figure 7Figure $7 \mathrm{~b}$ shows the deconvolution of the $\mathrm{O} 1 \mathrm{~s}$ spectra into two bands at $529.1 \mathrm{eV}$ and $531.6 \mathrm{eV}$ respectively. However, only the former band (with a $0.75 \mathrm{eV}$ chemical shift) was observed in the hydrothermally prepared sample. The first band relates to lattice oxygen $\left(\mathrm{O}^{2-}\right)$, while the latter relates to adsorbed surface oxygen or hydroxyl groups $\left(\mathrm{OH}^{-}\right)[13,31]$. The results summarised in Table 3Fable 3 show that the ratio of hydroxyl group to lattice oxygen increased with ultrasonic irradiation but decreased with hydrothermal synthesis. This indicates that the surface concentration of the $\mathrm{OH}^{-}$group is least for the hydrothermal process and greatest in the ultrasonic mediated process. This is consistent with findings from $\mathrm{CO}_{2}-\mathrm{TPD}$ analysis. This can be ascribed to the boosted crystallization of the metal oxides due to enhanced transport properties of supercritical water, a characteristic of the hydrothermal process [32]. Supplementary documents (Tables S1 and S2) support this finding. This hypothesis is made visible with the aid of the EDX spectroscopy (see supplementary documents, Figure S1), which shows the enhanced dispersion of the metals $\left(\mathrm{Al}^{3+}\right.$ and $\left.\mathrm{Mg}^{2+}\right)$ across the adsorbent. The high concentration of lattice oxygen in the hydrothermal route can be associated to the increased $\mathrm{Mg}$ content in the form of $\mathrm{Mg}$-O-Mg groups or $\mathrm{Mg}^{2+}-\mathrm{O}^{2-}$ ion pairs [33]. 
$\mathrm{CO}_{2}$-TPD analysis was used to measure the surface basicity of the synthesised LDHs (Figure

Formatted: Font: Not Bold

6Figure 8). Surface basicity increases with increase in desorption temperature [6]. As shown in Figure 6Figure $8 \mathrm{a}$, the desorption of $\mathrm{CO}_{2}$ basically occurs at overlapping peaks of $270{ }^{\circ} \mathrm{C}(\alpha)$ and

Formatted: Font: Not Bold $363{ }^{\circ} \mathrm{C}(\beta), 474{ }^{\circ} \mathrm{C}(\gamma)$ and $569{ }^{\circ} \mathrm{C}(\delta)$. At low temperatures $\left(<400{ }^{\circ} \mathrm{C}\right)$, desorption of $\mathrm{CO}_{2}$ is related to released $\mathrm{CO}_{2}$ from bicarbonates formed on weak and medium basic sites [10]. These weak sites are characteristics of the Brønsted $\mathrm{OH}^{-}$group which are observed to be more prominent in the conventional and ultrasonic mediated routes [6] (Figure 6Figure 8b). This corresponds with Formatted: Font: Not Bold reported literatures validating that more weak and moderate basic sites are formed with the aid of ultrasonic irradiation [13]. Nonetheless, $\mathrm{HPH}-\mathrm{LDH}$ desorbed $\mathrm{CO}_{2}$ at intermediate/high temperature states $\left(>400{ }^{\circ} \mathrm{C}\right)$. These are strong basic sites and relate to bi-dentate and mono-dentate carbonates adsorbed on low coordinative unsaturated lattice oxygen $\left(\mathrm{O}^{2-}\right)$. This tends to agree with XPS and EDX results. A practical application of the basicity of this material vary from the recovery of $\mathrm{CO}_{2}$ from flue gases at low basic strength (low temperature level) to higher basic strength (high temperature level) processes like sorption enhanced hydrogenation. The latter has the advantage of ease of regeneration at low temperature gradients [6]. 

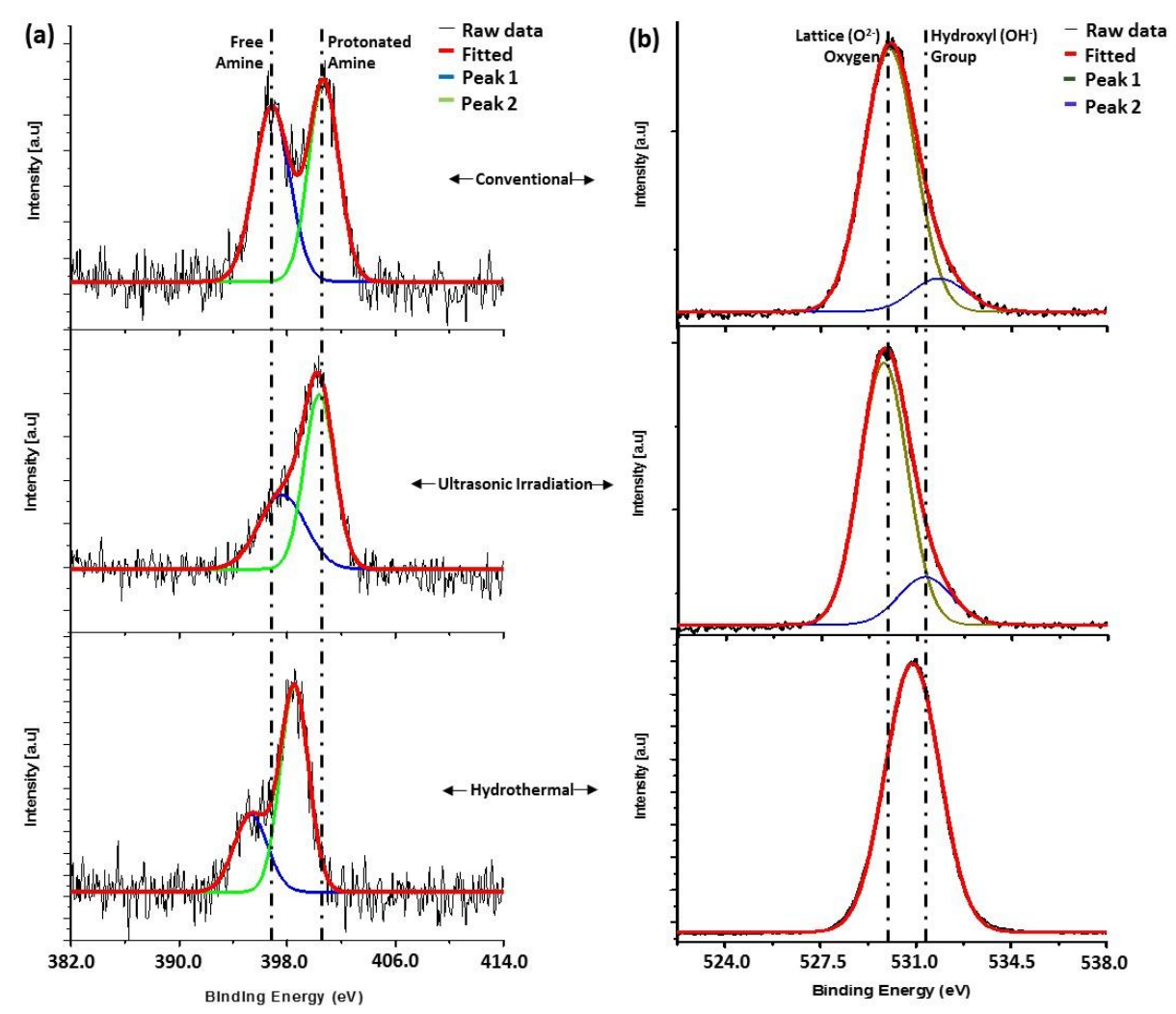

Figure 7: XPS spectra of conventionally-, ultrasonically-and hydrothermally-prepared LDHs for (a) $N 1 \mathrm{~s}$ and (b) $O 1 \mathrm{~s}$
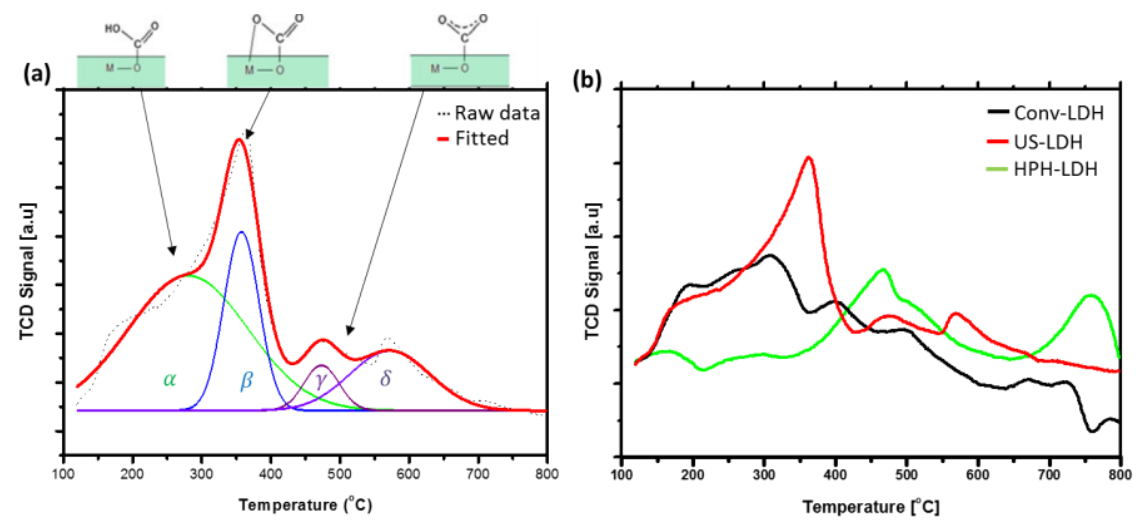

Figure 68: $\mathrm{CO}_{2}-\mathrm{TPD}$ of synthesised $\mathrm{LDH}$ (a) Deconvolution of the $\mathrm{CO}_{2}-\mathrm{TPD}$ of UH-LDH and (b) Comparison of $\mathrm{CO}_{2}-\mathrm{TPD}$ of Conv-LDH, US-LDH and HPH-LDH. 
To further understand the surface chemical content of the adsorbent, the amino functional group necessary for adsorption of $\mathrm{CO}_{2}$ was quantified using EDX results (See supplementary data Table S1). Table 4 shows the elemental analysis of each prepared sample using the EDX results from Table S1. As shown in Table 4, the amine loading was more effective using ultrasonic mediated route with $2.22 \mathrm{mmol} / \mathrm{g}$ as against 1.82 and $0.46 \mathrm{mmol} / \mathrm{g}$ for ultrasonic-assisted hydrothermal and the conventional route respectively. However, after functionalization of the adsorbent, the amine loading increased to $4.71,5.24$ and $5.26 \mathrm{mmol} / \mathrm{g}$ for conventional, ultrasonic and ultrasonicassisted hydrothermal synthesis respectively. This is observed to be a significant improvement compared to those results reported in literatures as documented in supplementary Table S3.

\subsection{Impact of preparation route on structural configuration}

Figure 7Figure 9 shows the XRD analysis of synthesised LDHs. Typical Mg-Al LDH exhibits three separate peak regions: (a) high angle region $\left(2 \theta>60^{\circ}\right)$ consisting of $h k 0$ and $h k l$ reflections attributed to the ordering within the metal hydroxide layers, (b) the mid-angle region $\left(30^{\circ}<2 \theta<\right.$ $60^{\circ}$ ) attributed to non-basal reflection $O k l$ whose locations are characteristic of the structural disorder and, (c) the low angle region $\left(2 \theta<30^{\circ}\right)$ consisting of the $00 l$ and $h 00$ basal reflections characteristic of the size of intercalated anion_[34]. These XRD reflections have been indexed according to the hexagonal lattice with rhombohedral symmetry (space group: R3m) [34-36]. A brief look from the figure indicates that the intensity of all reflections increased with the hydrothermal process. This is associated with an increase in interlayer water molecule and partial hydroxylation of double hydroxide sheets [37]. For the high angle region, peak at $2 \theta=60^{\circ}$ containing overlapping reflections of (110) and (113) attributed to interlayer spacing indicates that the hydrothermal route increases interlayer spacing. This is reported to be associated with the presence of oxygen-containing functional groups [38], which is observed to be highest in the hydrothermal synthetic method due to high metallic deposition and dispersion. Hydrothermal route is considered an important synthetic method for the preparation of highly dispersed heterogeneous materials. This is due to its tendency to dissolve and recrystallize materials at high temperature and pressure [39]. The 200 lattice peak reflection explains the crystal size of the material and its intensity is dependent on chemical composition and molar $\left(\mathrm{M}^{2+} / \mathrm{M}^{3+}\right)$ ratio [40]. Consequently, the strength of basic sites should vary with the peak intensity, thus validating the effect of deposition 
and dispersion of divalent and trivalent cations on basic strength. For the low and mid-angle region, the increase in peak intensities can be attributed to change in configuration of the interlayer nitrate from single flat-lying molecular layer to multiple layers and with different orientation [35, 41]. To this effect, using the results of X-ray diffraction and polarized attenuated total reflection FTIR spectroscopy, Wang and Wang [41] explained that the change in orientation of interlayer nitrate anions is dependent on chemical composition of LDH. This corresponds with XPS and TPD results.

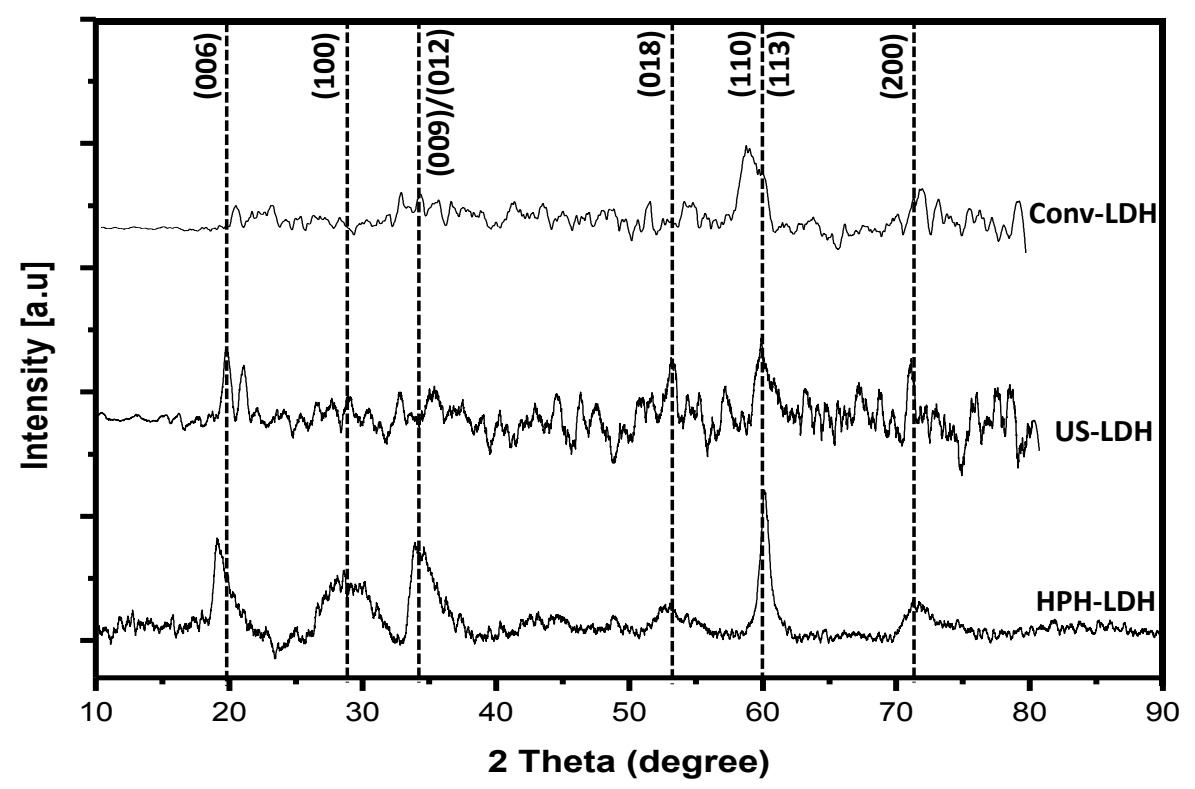

Figure 79: X-ray diffraction patterns of synthesised Mg/Al LDHs via conventional co-precipitation (Conv-LDH), ultrasonic irradiation (US-LDH) and sonochemical-assisted hydrothermal route (HPH-LPH)

\subsection{Impact of preparation route on thermal stability}

The thermal stability of the prepared samples were obtained using Netzsch STA 449 F3 TGA from $25{ }^{\circ} \mathrm{C}$ to $1000{ }^{\circ} \mathrm{C}$ with a $10{ }^{\circ} \mathrm{C} / \mathrm{min}$ ramp. Figure 8 Figure 10 shows the TGA profiles for thermal Formatted: Font: Not Bold disintegration of the prepared samples. Three distinct temperature phases were observed for material disintegration: $\mathrm{T}<\sim 150{ }^{\circ} \mathrm{C}, \sim 150<\mathrm{T}<\sim 750{ }^{\circ} \mathrm{C}$ and $\sim 750{ }^{\circ} \mathrm{C}<\mathrm{T}$. The first phase of weight loss $\left(\mathrm{T}<\sim 150{ }^{\circ} \mathrm{C}\right)$ is attributed to the loss of interstitial water molecules. While for the second 
phase $\left(\sim 150<\mathrm{T}<\sim 750{ }^{\circ} \mathrm{C}\right)$, the decomposition can be ascribed to the dehydroxylation and breakdown of the organic alkyl chain of the LDH. The observed irregular decomposition curve in this stage may indicate the occurrence of an uneven bonding structure, resulting to multi-stage dehydroxylation processes. The final weight loss $\left(\sim 750{ }^{\circ} \mathrm{C}<\mathrm{T}\right)$ results from the decomposition of the sulphate species residuum [10]. From Figure 8Figure 10a-c, it can be inferred that the thermal strength of HPH-LDH is higher than that of the conventional and ultrasonically prepared samples with a total weight loss of 68.98 amd $68.12 \%$ respectively, when compared to $10.56 \%$ of $\mathrm{HPH}$ LDH as tabulated in Table S4 (supplementary document). For the first phase, the moisture content decreased significantly from 8.35 to $3.41 \%$ as the preparation process changed from conventional to hydrothermal. A more drastic reduction was experienced with the interstitial moisture content (second phase). This can be attributed to the enhanced bonding introduced by the hydrothermal process forming multi-layers as estimated from the XRD results. The third degradation phase infers that ultrasonic process is more likely to deposit more sulphate species, while the hydrothermal process is the least to do so. This is corresponds to the results obtained from XPS analysis (Table S2, supporting document). This indicates that the adoption of the hydrothermalroute may be beneficial to the synthesis of a more stable material than the ultrasonic and conventional method.

After introducing amine modification of the samples, the decomposition curves clearly show different behaviour compared with that of the untreated LDHs prepared by the same method. The curves display a well-defined three phase decomposition steps unlike the untreated ones, as can be seen from Figure 8Figure 10(d-f). Within the same temperature range as that of the LDHs, the MEA-treated LDHs showed higher moisture content than the unprocessed ones except for the hydrothermal samples. This can be caused by the presence of leftover MEA molecules after the extraction process, which may have been strongly bonded to the hydrothermal samples resulting to the increase in weight loss in the second and third phase. However, for the ultrasonic and conventional route, the weight losses in the second and third phase reduced significantly, benefitting to production of a more stable material than the pure $\mathrm{LDH}$. This can be explained by the reduced presence of the surfactant after the amine extraction. Showing from the total weight loss, it can be concluded that the thermal strength of the hydrothermal samples (total weight loss: 
$38.17 \%$ ) even after amine extraction is better than that of the conventional and ultrasonically prepared samples (total weight loss: 52-53\%).

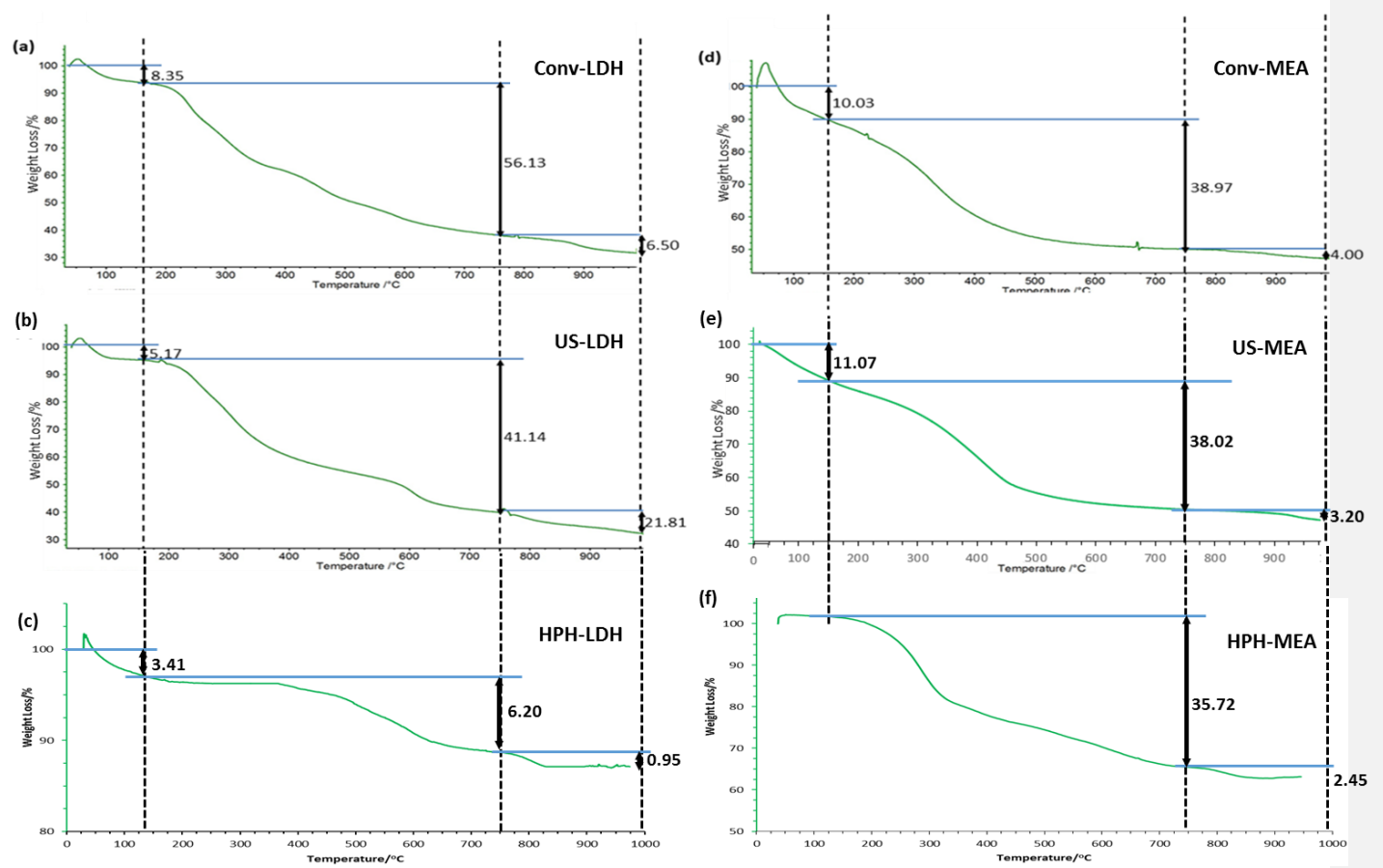

Figure 810: TGA curves comparing thermal stabilities of LDHs prepared via conventional and ultrasonic irradiation: (a) Conv-LDH, (b) UH-LDH and (c) HPH-LDH; as well as with amine modified LDHs: (d) Conv-MEA (e) UH-MEA and (f) HPH-MEA

\subsection{Impact of preparation route on effective amine efficiency, $\mathrm{CO}_{2}$ uptake, regeneration efficiency and cyclic efficiency}

The average $\mathrm{CO}_{2}$ uptake for the various synthesis route is shown in Figure 9Figure 14. The results show a reduction in $\mathrm{CO}_{2}$ uptake for the US-LDH when compared to the conventional, Conv-LDH. This corresponds to results from previous study [10]. However, an insignificant increase in $\mathrm{CO}_{2}$ uptake was observed for the hydrothermally prepared sample, HPH-LDH. Despite the enhanced textural properties of HPH-LDH, the adsorption capacity of this adsorbent showed no appreciable improvement. However, after amine extraction, the amine loading of adsorbents increased for all 
considered routes. This is part due to the exfoliation of the surfactant (SDS) (shown in Table 4Table 4) which varied from ca. 99\%, $98 \%$ and $81 \%$ in the conventional, ultrasonic and hydrothermal processes respectively. As a result, this impacted on the effective amine loading and efficiency of the adsorbent. The effective amine loading was computed as the difference in amine loading before and after amine extraction, whereas effective amine efficiency was computed as the ratio of the difference in $\mathrm{CO}_{2}$ adsorbed before and after amine extraction to effective amine loading. HPH-MEA showed the highest effective amine efficiency of 0.83 compared to 0.24 and 0.15 for US-MEA and Conv-MEA respectively. This can be partly related to the improved deprotonation of protonated amino groups resulting from enhanced transport properties, characteristic of the hydrothermal process [32]. This expounds the impact of sonication and hydrothermal pre-treatment in deprotonating protonated and/or probable dispersion of amino sites during LDH-amine modification. Consequently, the adsorption capacity of HPH-LDH increases from $0.88-3.75 \mathrm{mmol} / \mathrm{g}$ when compared to the increase of Conv-LDH from $0.82-1.45 \mathrm{mmol} / \mathrm{g}$ (supplementary document, Table S5). This indicates that $\mathrm{CO}_{2}$ uptake was stimulated more by chemisorption rather than physisorption. This can be attributed to the difference in basic strength of the prepared adsorbents.

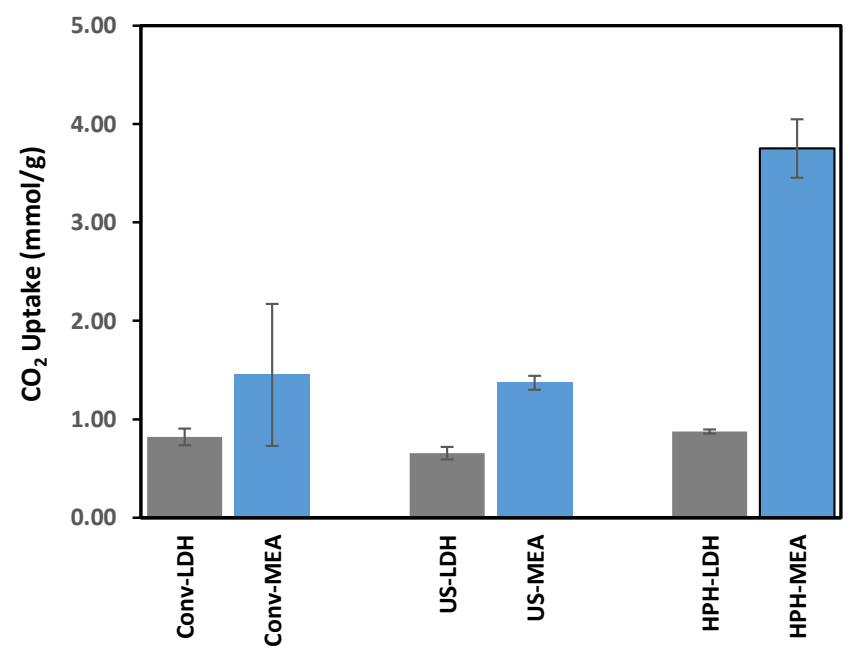

Figure 911: Average $\mathrm{CO}_{2}$ Uptake of $\mathrm{LDH}$ and amine-modified $\mathrm{LDH}$ prepared via the different routes 
Thermal swing regeneration in an atmosphere of $\mathrm{N}_{2}$ was adopted for the regeneration of the amine modified adsorbent. A typical example of the regeneration profile is shown in Figure $\underline{S} 2$ of supplementary document. In this figure, the effect of the preparation route on the cyclic sorption capacity of the adsorbent is presented. Amount of $\mathrm{CO}_{2}$ desorbed was measured after the first adsorption cycle prior to the second adsorption process. Consecutively, the regeneration and cyclic efficiencies of the adsorbents were calculated (supplementary document, Table S5). From the tabulated results in the figure, it is observed that HPH-MEA has the highest regeneration efficiency of $93 \%$. This is a significant change when compared to $78 \%$ and $86 \%$ regeneration efficiencies for US-MEA and Conv-MEA respectively. However, the cyclic efficiency (computed as the percentage ratio of $\mathrm{CO}_{2}$ uptake from $2^{\text {nd }}$ cycle to $1^{\text {st }}$ cycle) of US-MEA is $76 \%$ compared to $60 \%$ and 53\% for Conv-MEA and HPH-MEA respectively. Analysing the cyclic and regenerated efficiencies, it can be projected that the ultrasonic mediated synthesis route has an effective adsorption potential than the other two processes. It is observed that of the $78 \%$ regenerated active site, approx. $76 \%$ of this active site underwent the $2^{\text {nd }}$ adsorption process. This is lesser in ConvMEA and HPH-MEA respectively. The degradation in the cyclic adsorption efficiencies of these samples can be ascribed to the secondary reactions occurring between the amino group and $\mathrm{CO}_{2}$ as elaborated in previous study [10]. This implies that the ultrasonic mediated route is more suitable for the synthesis of long term effective $\mathrm{CO}_{2} \mathrm{LDH}$ adsorbents. However, the hydrothermal process provides a significant change in sorption capacity of the adsorbent. Optimization of these two processes will contribute immensely to both sorption efficiency and effectiveness.

\subsection{Comparison of kinetic models}

From simulation results, the experimental kinetic uptake data for the samples synthesized from the different synthesis routes are summarized in Table 5Fable 5. Comparative plots of these data using the kinetic models discussed in the previous section are shown in Figure 10Figure 12. The results show that the double exponential model produced the best fit for the experimental data (considering the $\mathrm{R}^{2}$ values). This unlike the pseudo first and second order model, is explained to account for the heterogeneity of the adsorbent's surface [16]. In other words, it describes the adsorption mechanism to comprise of two different adsorption sites. Consequently, elucidating the occurrence of more than one reaction mechanism [42]. Therefore, the double exponential model can be explained to be of best fit of the three models because it possibly accounts for both 
chemisorption and physisorption of $\mathrm{CO}_{2}$. Rate constants $\mathrm{k}_{\mathrm{i}=1,2}$ are the reaction rate coefficients for the respective adsorption mechanisms. The simulated results showed no particular variation of these parameter with the preparation route. The pre-exponential factors, $A_{i=1,2}$, indicate the weight coefficient that reflects the share of each adsorption mechanism [17]. From the tabulated results, it is observed that the second adsorption mechanism (indicated by $\mathrm{i}=2$ ) contributed a greater share towards $\mathrm{CO}_{2}$ adsorption in Conv-LDH. This gradually reduced in US-LDH and was approximately evenly distributed between the two mechanisms in HPH-LDH.
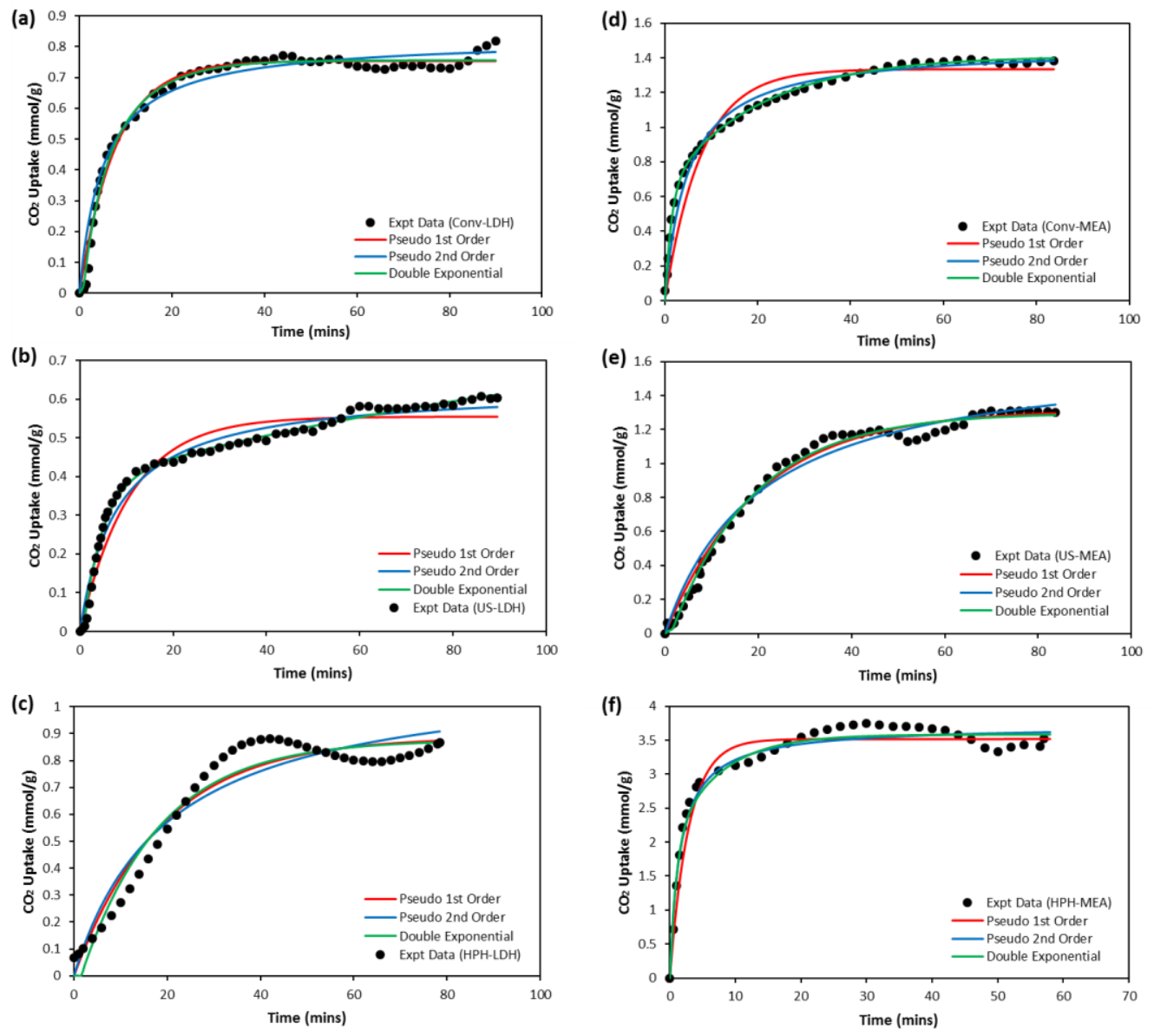
Figure 1012: Comparison of different kinetic models with experimental data generated for adsorption kinetics of $\mathrm{CO}_{2}$ on (a) Conv- $\mathrm{LDH}$, (b) US-LDH, (c) HPH-LDH, (d) Conv-MEA, (e) US$M E A$ and $(f) H P H-M E A$ under and $55^{\circ} C$ and $1 \mathrm{~atm}$

Pseudo first and second order model also provided a relatively good fit for the experimental results. However, comparing to pseudo second order model, pseudo first order provided a better fit for both Conv-LDH and HPH-LDH experimental data. This model better explains low surface coverage adsorption [43] whereas the pseudo second order model accounts for adsorption by chemisorption or adsorption at high adsorbate loadings [44]. Hence, it is logical to attribute the fitting of the second order model to US-LDH experimental data to the high amine loading. Considering the kinetic parameters, $\mathrm{k}_{\mathrm{f}}$ and $\mathrm{k}_{\mathrm{s}}$, which depicts the time scale for the process to reach equilibrium [17]. Inverse of these parameters indicates the time required for the process to attain equilibrium. As shown in Table 5Table 5, the $\mathrm{k}_{\mathrm{f}, \mathrm{s}}$ decreased in the following trend: conventional > ultrasonic > hydrothermal. This portrays that conventionally prepared LDH tends to attain equilibrium quicker than samples prepared from the other routes. Although pseudo first and second order models are associated with surface reaction control systems [17], the former was also derived by assuming film diffusion (FD) control [45] whereas the later simulates intra-particle diffusion (IPD)-driven kinetics of sorption for systems with both plane and spherical sorbent particles [46]. Consequently, it is likely to assume that IPD is more promoted in US-LDH.

After amine extraction, the double exponential model was also of best fit for the obtained experimental data. However, the reverse trend was observed for the share of each adsorption mechanisms. Summarised results (Table 5Table 5) show that both adsorption mechanism Formatted: Font: Not Bold approximately contributed evenly in the adsorption process in Conv-MEA and US-MEA; but the second mechanism dominated in HPH-MEA. The nature of these mechanisms are undetermined in this study but can be seen to give an insight on the adsorption trend of each sample. Nonetheless, correlating these findings with characterization results, the first adsorption mechanism (indicated by $i=1$ ) can be inferred to be associated with the physical textural properties of the adsorbent. Subsequently, the enhanced textural properties via ultrasonic and hydrothermal treatment resulted to the increase in the share of the first adsorption mechanism. However, after amine extraction, the second mechanism, assumed to be characterized by chemisorption, increased in share in the 
following trend: Conv-MEA < US-MEA < HPH-MEA. This can be attributed to the enhanced amine loading and effective amine efficiency.

\section{CONCLUSION}

A detailed comparison study of the impact of ultrasonic and hydrothermal pre-treatment on the basic strength, textural characteristics, effective amine efficiency, $\mathrm{CO}_{2}$ adsorption and regeneration capacities of $\mathrm{Mg}-\mathrm{Al} \mathrm{LDH}$ has been conducted. In addition, the contribution of these preparation routes to the thermal stability and reaction kinetics was discussed. The results obtained using the kinetic models clearly indicate that prior to amine extraction, the adsorption of $\mathrm{Mg}-\mathrm{Al} \mathrm{LDH}$ adsorbent is mostly facilitated by physisorption while adsorption is mainly promoted by chemisorption after amine extraction. The conclusions reached as the result of the present study can be summarised:

(1) Characterization results show that the ultrasonic-assisted hydrothermal route immensely improves the porosity and surface morphology of the adsorbent. Furthermore, the surface chemical content was characterised with improved metal-ion distribution, hence increasing the basic strength of the adsorbent. This strongly contributes to the improved adsorption and regeneration capacities, as well as the thermal stability of the adsorbent.

(2) The reason that the ultrasonic-assisted hydrothermal treatment can effectively promote the adsorption capacity of the adsorbent can be partially attributed to the ascent in the amount of strong basic sites $\left(\mathrm{O}^{2-}\right)$ and subsequent decrease in both moderate $(\mathrm{M}-\mathrm{O})$ and weak $\left(\mathrm{OH}^{-}\right.$groups)

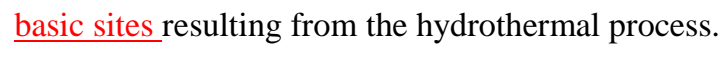

(3) The cyclic adsorption efficiency of the sonochemical process is better than that of both hydrothermal and conventional preparation routes. The sonochemical route shows the potential for enhanced amine loading and cyclic efficiency, thus providing a more suitable and economical approach for synthesis of double layered hydroxides. 


\section{ACKNOWLEDGEMENT}

This study was conducted at the International Doctoral Innovation Centre (IDIC). The authors would like to acknowledge the financial support from National Natural Science Foundation of China (NSFC) (Grant No. 21576141) and Zhejiang Provincial Natural Science Foundation (Grant No. LY15B060001) and Ningbo Key Research Project 'Absorption/catalytic technologies for the simultaneous removal of multi-pollutants from flue gas at power stations' (Grant No. 1012B10042), and from Ningbo Science and Technology Bureau, The University of Nottingham, Ningbo Education Bureau and China's MoST. The study is also partly supported by EPSRC (Grant no. EP/G037345/1). In addition,

\section{References}

[1] J. Wang, L.A. Stevens, T.C. Drage, C.E. Snape, J. Wood, Preparation and CO2 adsorption of amine modified layered double hydroxide via anionic surfactant-mediated route, Chemical Engineering Journal, 181-182 (2012) 267-275.

[2] J.D. Figueroa, T. Fout, S. Plasynski, H. McIlvried, R.D. Srivastava, Advances in CO2 capture technology-The U.S. Department of Energy's Carbon Sequestration Program, International Journal of Greenhouse Gas Control, 2 (2008) 9-20.

[3] M. Szabados, R. Mészáros, S. Erdei, Z. Kónya, Á. Kukovecz, P. Sipos, I. Pálinkó, Ultrasonically-enhanced mechanochemical synthesis of CaAl-layered double hydroxides intercalated by a variety of inorganic anions, Ultrasonics Sonochemistry, 31 (2016) 409-416.

[4] F. Cavani, F. Trifirò, A. Vaccari, Hydrotalcite-type anionic clays: Preparation, properties and applications, Catalysis Today, 11 (1991) 173-301.

[5] N.D. Hutson, B.C. Attwood, High temperature adsorption of $\mathrm{CO} 2$ on various hydrotalcite-like compounds, Adsorption, 14 (2008) 781-789.

[6] D.I. Ferrer, Supported Layered Double Hydroxides as CO2 Adsorbents for Sorption-enhanced H2 Production, Chemical Engineering, Imperial College, London, United Kingdom, Springer International Publishing Switzerland, 2016, pp. 209.

[7] L.H. Chagas, G.S.G. De Carvalho, W.R. Do Carmo, R.A.S. San Gil, S.S.X. Chiaro, A.A. Leitão, R. Diniz, L.A. De Sena, C.A. Achete, MgCoAl and NiCoAl LDHs synthesized by the hydrothermal urea hydrolysis method: Structural characterization and thermal decomposition, Materials Research Bulletin, 64 (2015) 207-215.

[8] Y. Gao, Z. Zhang, J. Wu, X. Yi, A. Zheng, A. Umar, D. O'Hare, Q. Wang, Comprehensive

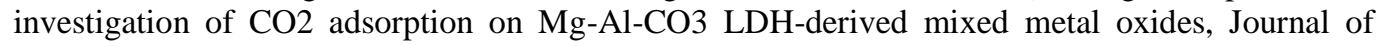
Materials Chemistry A, 1 (2013) 12782-12790.

[9] J.I. Di Cosimo, V.K. Díez, M. Xu, E. Iglesia, C.R. Apesteguía, Structure and Surface and Catalytic Properties of Mg-Al Basic Oxides, Journal of Catalysis, 178 (1998) 499-510.

[10] C.I. Ezeh, X. Huang, X. Yang, C.-g. Sun, J. Wang, Sonochemical surface functionalization of exfoliated LDH: Effect on textural properties, $\mathrm{CO} 2$ adsorption, cyclic regeneration capacities and 
subsequent gas uptake for simultaneous methanol synthesis, Ultrasonics Sonochemistry, 39 (2017) 330-343.

[11] D.P. Debecker, E.M. Gaigneaux, G. Busca, Exploring, tuning, and exploiting the basicity of hydrotalcites for applications in heterogeneous catalysis, Chemistry-A European Journal, 15 (2009) 3920-3935.

[12] Y. Mu, Y. Zhang, J. Fan, C. Guo, Effect of ultrasound pretreatment on the hydrothermal synthesis of SSZ-13 zeolite, Ultrasonics Sonochemistry, 38 (2017) 430-436.

[13] S. Zhao, H. Yi, X. Tang, F. Gao, Q. Yu, Y. Zhou, J. Wang, Y. Huang, Z. Yang, Enhancement effects of ultrasound assisted in the synthesis of $\mathrm{NiAl}$ hydrotalcite for carbonyl sulfide removal, Ultrasonics Sonochemistry, 32 (2016) 336-342.

[14] H.J. Jang, C.H. Lee, S. Kim, S.H. Kim, K.B. Lee, Hydrothermal Synthesis of K2CO3Promoted Hydrotalcite from Hydroxide-Form Precursors for Novel High-Temperature CO2 Sorbent, ACS Applied Materials \& Interfaces, 6 (2014) 6914-6919.

[15] I. Khan, A.A.M. Ibrahim, M. Sohail, A. Qurashi, Sonochemical assisted synthesis of $\mathrm{RGO} / \mathrm{ZnO}$ nanowire arrays for photoelectrochemical water splitting, Ultrasonics Sonochemistry, 37 (2017) 669-675.

[16] S. Loganathan, M. Tikmani, A. Mishra, A.K. Ghoshal, Amine tethered pore-expanded MCM41 for $\mathrm{CO} 2$ capture: Experimental, isotherm and kinetic modeling studies, Chemical Engineering Journal, 303 (2016) 89-99.

[17] K.L. Tan, B.H. Hameed, Insight into the adsorption kinetics models for the removal of contaminants from aqueous solutions, Journal of the Taiwan Institute of Chemical Engineers, 74 (2017) 25-48.

[18] M.P. Bernardo, F.K.V. Moreira, L.A. Colnago, C. Ribeiro, Physico-chemical assessment of [Mg-Al-PO4]-LDHs obtained by structural reconstruction in high concentration of phosphate, Colloids and Surfaces A: Physicochemical and Engineering Aspects, 497 (2016) 53-62.

[19] A. Laskowska, M. Zaborski, G. Boiteux, O. Gain, A. Marzec, W. Maniukiewicz, Effects of unmodified layered double hydroxides MgAl-LDHs with various structures on the properties of filled carboxylated acrylonitrile-butadiene rubber XNBR, European Polymer Journal, 60 (2014) 172-185.

[20] H.W. Olfs, L.O. Torres-Dorante, R. Eckelt, H. Kosslick, Comparison of different synthesis routes for $\mathrm{Mg}-\mathrm{Al}$ layered double hydroxides ( $\mathrm{LDH})$ : Characterization of the structural phases and anion exchange properties, Applied Clay Science, 43 (2009) 459-464.

[21] L. Dong, C. Ge, P. Qin, Y. Chen, Q. Xu, Immobilization and catalytic properties of candida lipolytic lipase on surface of organic intercalated and modified MgAl-LDHs, Solid State Sciences, 31 (2014) 8-15.

[22] B. Li, Y. Zhang, X. Zhou, Z. Liu, Q. Liu, X. Li, Different dye removal mechanisms between monodispersed and uniform hexagonal thin plate-like MgAl-CO32--LDH and its calcined product in efficient removal of Congo red from water, Journal of Alloys and Compounds, 673 (2016) 265 271.

[23] W. Lv, L. Yang, B. Fan, Y. Zhao, Y. Chen, N. Lu, R. Li, Silylated MgAl LDHs intercalated with $\mathrm{MnO} 2$ nanowires: Highly efficient catalysts for the solvent-free aerobic oxidation of ethylbenzene, Chemical Engineering Journal, 263 (2015) 309-316.

[24] H. Nina, A.R. Barron, BET Surface Area Analysis of Nanoparticles, Rice University, Houston TX, 2011.

[25] S. Brunauer, L.S. Deming, W.E. Deming, E. Teller, On a Theory of the van der Waals Adsorption of Gases, J. Am. Chem. Soc., 62 (1940) 1723-1732. 
[26] K.S.W. Sing, D.H. Everett, R.A.W. Haul, L. Moscou, R.A. Pierotti, J. Rouquerol, S. T., Reporting Physisorption Data for Gas/Solid Systems with Special Reference to the Determination of Surface Area and Porosity, Pure \& Appl. Chem., 57 (1985) 603-619.

[27] T. Adschiri, Y. Hakuta, K. Arai, Hydrothermal Synthesis of Metal Oxide Fine Particles at Supercritical Conditions, Industrial and Engineering Chemistry Research, 39 (2000) 4901-4907.

[28] H. Xu, B.W. Zeiger, K.S. Suslick, Sonochemical synthesis of nanomaterials, Chemical Society reviews, 42 (2013) 2555-2567.

[29] Y. Kong, G. Jiang, Y. Wu, S. Cui, X. Shen, Amine hybrid aerogel for high-efficiency CO2 capture: Effect of amine loading and CO2 concentration, Chemical Engineering Journal, 306 (2016) 362-368.

[30] R. Dolores, S. Raquel, G.-L. Adianez, Sonochemical synthesis of iron oxide nanoparticles loaded with folate and cisplatin: Effect of ultrasonic frequency, Ultrasonics Sonochemistry, 23 (2015) 391-398.

[31] E. Genty, J. Brunet, C. Poupin, S. Casale, S. Capelle, P. Massiani, S. Siffert, R. Cousin, Co-Al mixed oxides prepared via LDH route using microwaves or ultrasound: Application for catalytic toluene total oxidation, Catalysts, 5 (2015) 851-867.

[32] J. Otsu, Y. Oshima, New approaches to the preparation of metal or metal oxide particles on the surface of porous materials using supercritical water: Development of supercritical water impregnation method, J. of Supercritical Fluids, 33 (2005) 61-67.

[33] M. Manikandan, A.K. Venugopal, K. Prabu, R.K. Jha, R. Thirumalaiswamy, Role of surface synergistic effect on the performance of Ni-based hydrotalcite catalyst for highly efficient hydrogenation of furfural, Journal of Molecular Catalysis A: Chemical, 417 (2016) 153-162.

[34] N. Touisni, F. Charmantray, V. Helaine, C. Forano, L. Hecquet, C. Mousty, Optimized immobilization of transketolase from E. coli in MgAl-layered double hydroxides, Colloids and Surfaces B: Biointerfaces, 112 (2013) 452-459.

[35] S.-L. Wang, C.H. Liu, M.K. Wang, Y.H. Chuang, P.N. Chiang, Arsenate adsorption by $\mathrm{Mg} / \mathrm{Al}-\mathrm{NO} 3$ layered double hydroxides with varying the $\mathrm{Mg} / \mathrm{Al}$ ratio, Applied Clay Science, 43 (2009) 79-85.

[36] M. Bellotto, B. Rebours, O. Clause, J. Lynch, D. Bazin, E. Elkaïm, A Reexamination of Hydrotalcite Crystal Chemistry, The Journal of Physical Chemistry, 100 (1996) 8527-8534.

[37] H. Ji, W. Wu, F. Li, X. Yu, J. Fu, L. Jia, Enhanced adsorption of bromate from aqueous solutions on ordered mesoporous Mg-Al layered double hydroxides (LDHs), Journal of Hazardous Materials, 334 (2017) 212-222.

[38] S. Zhao, X. Meng, K. Zhu, F. Du, G. Chen, Y. Wei, Y. Gogotsi, Y. Gao, Li-ion uptake and increase in interlayer spacing of Nb4C3 MXene, Energy Storage Materials, 8 (2017) 42-48.

[39] H. Emadi, M. Salavati-Niasari, A. Sobhani, Synthesis of some transition metal (M: 25Mn, $27 \mathrm{Co}, 28 \mathrm{Ni}, 29 \mathrm{Cu}, 30 \mathrm{Zn}, 47 \mathrm{Ag}, 48 \mathrm{Cd}$ ) sulfide nanostructures by hydrothermal method, Advances in Colloid and Interface Science, DOI https://doi.org/10.1016/j.cis.2017.06.007.

[40] J.S. Valente, M. Sánchez-Cantú, E. Lima, F. Figueras, Method for Large-Scale Production of Multimetallic Layered Double Hydroxides: Formation Mechanism Discernment, Chemistry of Materials, 21 (2009) 5809-5818.

[41] S.-L. Wang, P.-C. Wang, In situ XRD and ATR-FTIR study on the molecular orientation of interlayer nitrate in $\mathrm{Mg} / \mathrm{Al}$-layered double hydroxides in water, Colloids and Surfaces A: Physicochemical and Engineering Aspects, 292 (2007) 131-138.

[42] L. Sciascia, M.L. Turco Liveri, M. Merli, Kinetic and equilibrium studies for the adsorption of acid nucleic bases onto K10 montmorillonite, Applied Clay Science, 53 (2011) 657-668. 
[43] R. Serna-Guerrero, A. Sayari, Modeling adsorption of $\mathrm{CO} 2$ on amine-functionalized mesoporous silica. 2: Kinetics and breakthrough curves, Chemical Engineering Journal, 161 (2010) 182-190.

[44] J.M. Borah, J. Sarma, S. Mahiuddin, Adsorption comparison at the $\alpha$-alumina/water interface: 3,4-Dihydroxybenzoic acid vs. catechol, Colloids and Surfaces A: Physicochemical and Engineering Aspects, 387 (2011) 50-56.

[45] W. Plazinski, Applicability of the film-diffusion model for description of the adsorption kinetics at the solid/solution interfaces, Applied Surface Science, 256 (2010) 5157-5163.

[46] W. Plazinski, J. Dziuba, W. Rudzinski, Modeling of sorption kinetics: the pseudo-second order equation and the sorbate intraparticle diffusivity, Adsorption, 19 (2013) 1055-1064. 
Supplementary Interactive Plot Data (CSV)
Click here to download Supplementary Interactive Plot Data (CSV): Supporting Document.docx

Supplementary Interactive Plot Data (CSV)
Click here to download Supplementary Interactive Plot Data (CSV): Supporting Document.docx

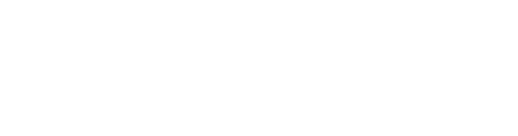

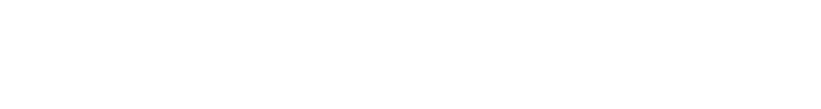

.

.

.

.

.

.

.

.

.

.

.

.

.

.

.

.

.

.

.

.

.

.

.

.

.

.

.

.

.

.

.

.

.

. 